\title{
Rapid Review of Water Knowledge for Pacific Small Islands Developing States
}

JUNE 2018 


\section{Acknowledgement \& disclaimer}

(C) 2017 International Bank for Reconstruction and Development / The World Bank

1818 H Street NW, Washington, DC 20433

Telephone: 202-473-1000; Internet: www.worldbank.org

This work is a product of the staff of The World Bank with external contributions. The findings, interpretations, and conclusions expressed in this work do not necessarily reflect the views of The World Bank, its Board of Executive Directors, or the governments they represent.

The World Bank does not guarantee the accuracy of the data included in this work. The boundaries, colors, denominations, and other information shown on any map in this work do not imply any judgment on the part of The World Bank concerning the legal status of any territory or the endorsement or acceptance of such boundaries.

Rights and Permissions

The material in this work is subject to copyright. Because The World Bank encourages dissemination of its knowledge, this work may be reproduced, in whole or in part, for noncommercial purposes as long as full attribution to this work is given.

Please cite the work as follows: Dahan S., 2018: Rapid Review of Water Knowledge for Pacific Small Islands Developing States. World Bank, Washington, DC.

Any queries on rights and licenses, including subsidiary rights, should be addressed to World Bank Publications, The World Bank Group, 1818 H Street NW, Washington, DC 20433, USA; fax: 202-522-2625; e-mail: pubrights@worldbank.org.

Cover design: Jean Franz, Franz \& Company, Inc. 


\section{Contents}

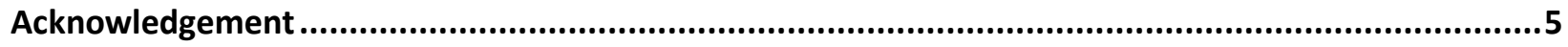

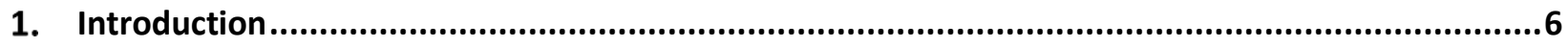

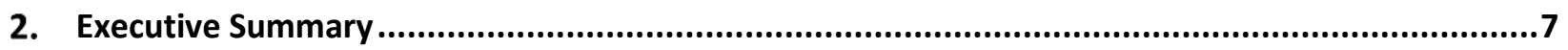

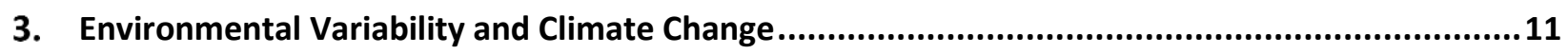

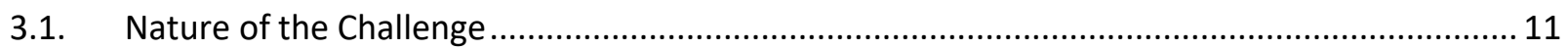

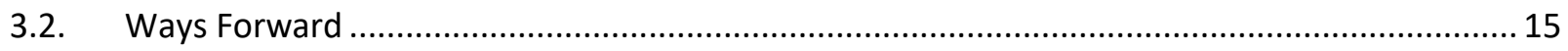

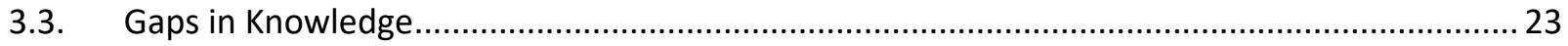

4. Water Sector Governance, Coordination, Integration, and Capacity .........................................24

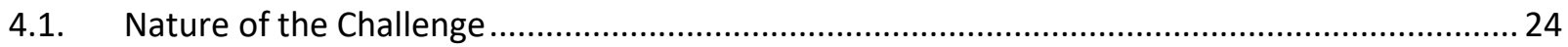

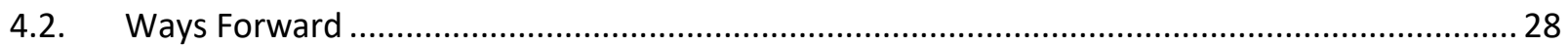

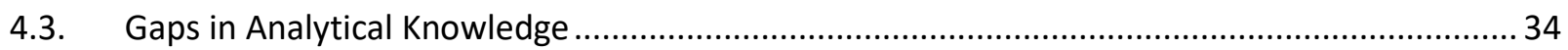

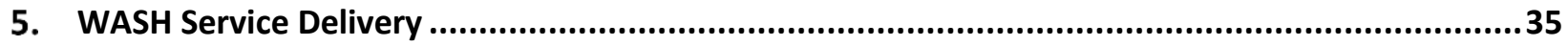

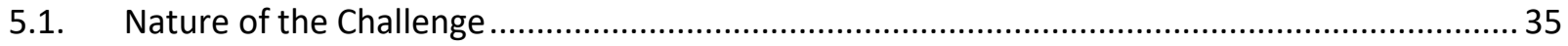

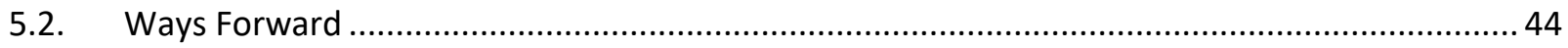

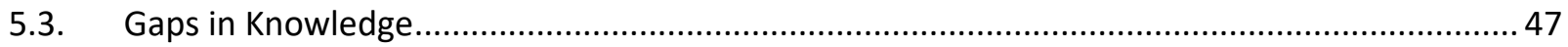

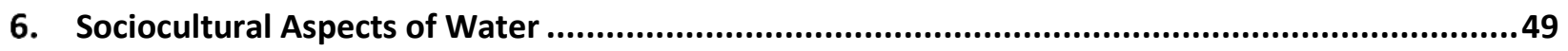

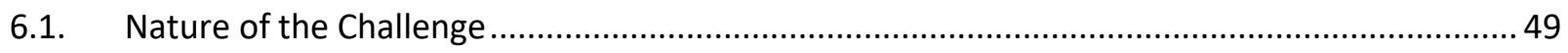

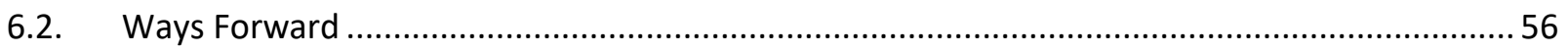

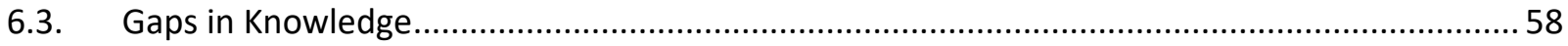

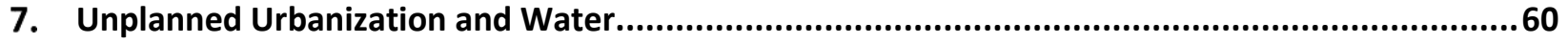

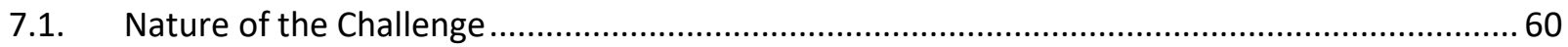

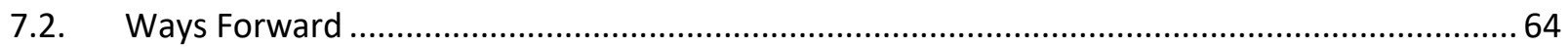

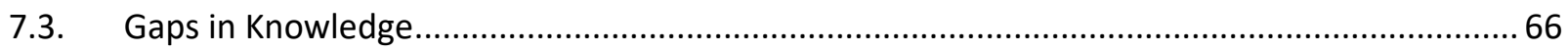

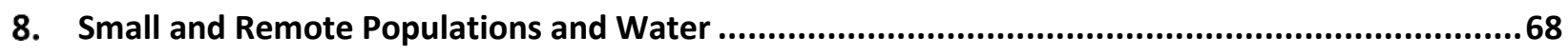

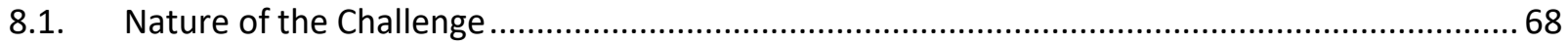

8.2. Lessons from Other Global Regions with Remote and Small Populations ............................... 70

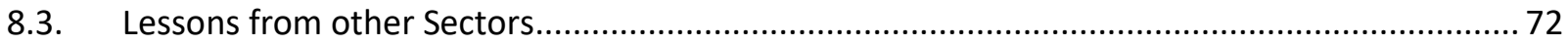

8.4. Ways Forward for Water Management and Services for Remote and Small Populations......... 74

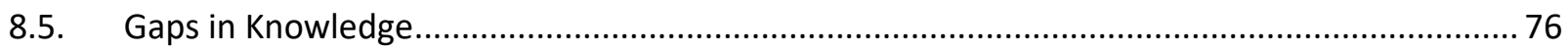

9. Water Data, Information, and Knowledge .............................................................................77

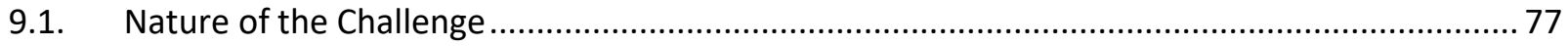

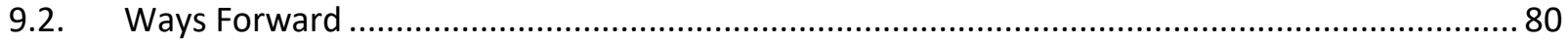




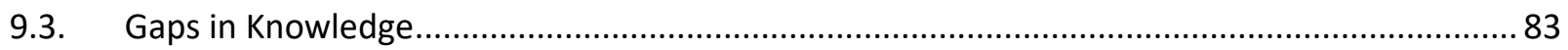

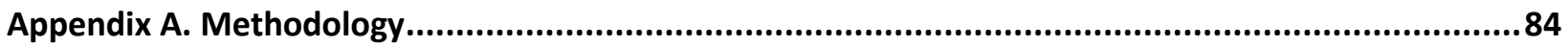

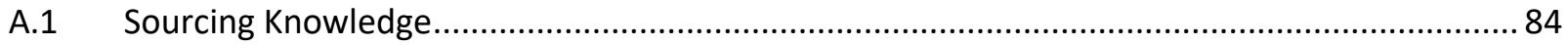

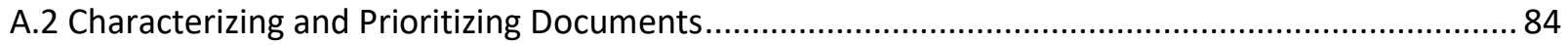

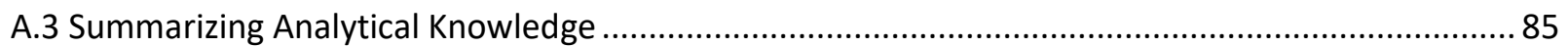

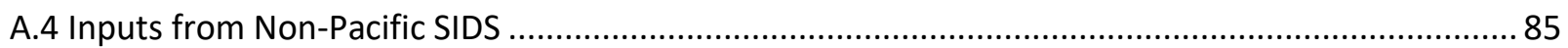

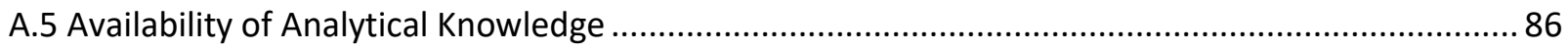

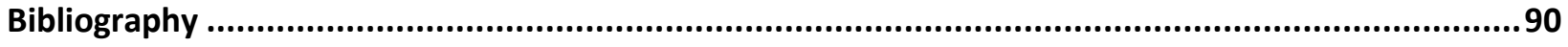




\section{Acknowledgement}

The report has been written by Regina Souter and Gemma Schuch from the International Water Centre. The report was commissioned by the World Bank Group's Water Global Practice. The Bank team, led by Stephane Dahan, worked closely with the International WaterCentre. The team comprised Edkarl Galing, Suneira Rana, and Meleesa Naughton.

The report benefited greatly from contributions from peer reviewers Habiba Gitay, Alexandre Danilenko, and Artessa Saldivar-Dali. This work benefited from overall guidance by Sudipto Sarkar.

Finally, the team is grateful to the Global Water and Sanitation Program (GWSP) for its support to the achievement of this work. 


\section{Introduction}

The objective of this report is to provide an overview of current knowledge related to the water sector ${ }^{1}$ across Small Island Developing States (SIDS) of the Pacific region. SIDS face a specific set of water, sanitation, and hygiene (WASH) challenges related to the extreme fragility of their water resources to anthropogenic impacts and climate change, the unique features of their water cycles, lack of scale, and isolation. Many of the modern water management paradigms applied elsewhere need careful reconsideration in that context. Consolidating a review of current knowledge, approaches, and best practices across the region can therefore be of high value to stimulate new thinking and prevent duplication of efforts. The reader will find captured in this report the findings and recommendations of development practitioners, researchers, policy makers who have left their mark over the past decades on the water, sanitation and hygiene sector in the Pacific region. Those can in turn help inform the design of future water, sanitation and hygiene development projects and programs, and the scoping of academic work.

This rapid study does not claim to have captured in a fully comprehensive manner the entire scope of knowledge on the subject, but with more than 500 relevant publications identified and reviewed, it most likely offers a reasonable overview of the current state of knowledge. To complement and put in perspective the analysis on Pacific SIDS, the study included reflections on of practice and knowledge in non-Pacific SIDS.

The first section of the report provides a summary of findings in terms of key knowledge areas and gaps, and proposes recommendations for future analytical work in the water sector in the Pacific. The following sections provide a more detailed review of current knowledge across seven key challenges identified as particularly critical in Pacific SIDS context: (i) environmental variability and change; (ii) sociocultural aspects; (iii) isolation and small scale; (iv) uncontrolled urbanization; (v) service delivery models; (vi) sector coordination, integration, and capacity; and (vii) data, information, and knowledge. Each topic is covered by a dedicated section describing the nature of the challenge, the identified ways forward, and knowledge gaps. Appendix A provides a description of the methodology used for this study.

${ }^{1}$ Unless indicated otherwise, the term water covers water supply, sanitation, hygiene, and water resource management. 


\section{Executive Summary}

The rapid review confirms that Pacific Small Island Developing States (SIDS) are socially, culturally, and environmentally diverse, with some features in common, and which collectively make them unique to other regions of the world:

- Small island countries have uniquely fragile water resources due to their small size, lack of natural storage, competing land use, and vulnerability to natural and anthropogenic hazards, including drought, cyclones, and urban pollution (with between 0.5 percent to 6.6 percent of gross domestic product (GDP) being lost annually to disasters).

- Rurally dominated populations are widely dispersed geographically as many small communities, while most capital cities include significant informal settlement populations and are subject to rapid urbanization (a more than 3 percent yearly increase in urban population growth in most Melanesian countries is projected between 2015 and 2020) (Mycoo and Donovan 2017).

- Water service providers face challenging constraints to sustaining water and wastewater provision due to the lack of both human and financial resource bases. Drivers of economic growth are limited: there are few export sectors and a large formal unemployment, which is higher for youths, women, and other disadvantaged groups, such as those with disabilities. Youth unemployment is estimated at 23 percent in the Pacific Islands region, rising to 62 percent in the Marshall Islands, compared to the global average of 12.6 percent (Wilson 2018).

- Water governance is highly complex due to the specific sociopolitical and cultural structures relating to traditional community, tribal, and interisland practices, rights, and interests. These are all interwoven with past colonial and "modern" practices and instruments. Most Pacific SIDS gained their independence from former colonial powers in the 1960s and 1970s.

- There are highly diverse sociocultural environments, varying geographically, but with urbanization creating multi-ethnic urban centres. Beyond obvious regional features across Melanesian, the Federated States of Micronesia, and Polynesian cultural areas, stark diversity can be found within countries. Papua New Guinea, the Solomon Islands, and Vanuatu stand among the world's top three countries in terms of linguistic diversity. ${ }^{2}$

Based upon the more than 500 sources reviewed, seven core challenges have emerged as critically affecting the achievement of sustainable water development, governance and management for Pacific SIDS. While all challenges are, to a certain extent, relevant across low-income (and upper-income) countries globally, most are particularly acute in the SIDS.

1. Environmental Variability and Climate Change. Urbanization and population growth have led to increased water demands and pollution across Pacific SIDS. There is often little regulation relating to water

${ }^{2}$ Calculated as the probability that two people of the country selected at random would have a different first language. See "Ethnologue," https://www.ethnologue.com/statistics/country. 
quality or source water protection and pollution control. These factors enhance water stress and SIDS' vulnerability to the effects of climate change, which include: increased rainfall intensity and seasonal variability, flood, drought, more intense cyclones, and sea level rise. Water resources in atoll islands are particularly vulnerable to climate variations and anthropogenic shocks. In high islands, cyclones and heavy rainfall events can damage water supply infrastructure or contaminate drinking water supplies. Extensive land clearing can impact spring flows, stream flows, and water supply infrastructure.

Despite having climate change adaptation (CCA) and disaster risk reduction (DRR) policy frameworks, many Pacific SIDS often remain relatively unprepared for flood and drought events. Efforts to increase disaster resilience and address environmental change include: (i) diversifying water sources, (ii) improving water resources monitoring, (iii) improving of water quality protection policies, drought management and integrated urban water management (IWRM), (iv) applying the WASH resilience concept, (v) integrating DRR and CCA policy frameworks, which have traditionally been distinct.

$\rightarrow$ Persistent knowledge gaps relate to the costs of climate adaptation options, data for risk management, the contribution of rainwater harvesting in urban areas, suitable wastewater treatment systems, and the potential for reuse. They also relate to rural and peri-urban household water management practices and strategies, how they may vary by season, and how they can impact vulnerability to climate change.

2. Water Sector Governance, Coordination, Integration, and Capacity. Failures in water management often stem from a lack of coordination across sectors and levels of the governance system. The environmental, social, economic and cultural aspects of water have not been brought under a single focus so that the gains from integrated management (efficiencies of planning and operation, management of resources, etc.) can be realized. This has been compounded by a frequent lack of (i) sector policies and regulations, (ii) clearly defined roles and responsibilities of government agencies, and (iii) coordination between donors and international organizations. The small and dispersed populations further impede efforts to build capacity and maintain functioning relationships within the sectors. Capacity can be built only with long-term phased approaches that tackle priority problems systematically. Knowledge gap areas include:

$\rightarrow$ Models to strengthen sector governance through national water apex bodies, with effective decentralization and community engagement, have not been well documented in the region.

$\rightarrow$ The success of regional support initiatives (pooling of expertise, regional training program, peer-topeer learning) is indicated by anecdotal evidence; further evaluation could guide future developments.

$\rightarrow$ Efforts to build capacity would be strengthened by country-wide capacity needs assessments, together with an assessment of existing capacity building mechanisms. Technical capacities should be developed within an IWRM framework. Building nontechnical capacity is also key and often neglected.

3. WASH Service Delivery. Only half of the population in the Pacific region use improved drinking water sources and one-third of the population use improved sanitation. Over the past decades, the pace of improvement has been very slow, driven largely, at a regional level, by fast population growth in Melanesian countries. Although several Pacific SIDS have achieved high levels of coverage with improved drinking water sources, there is little information about the quality, safety and resilience of services.

Recommendations related to urban utilities include (i) improving the management of water supply catchments and systems efficiency; and (ii) increasing water storage capacity and reliance on sources that 
are not subject to climate variability (e.g. desalination), (iii) engaging urban communities in demand management campaigns, and (iv) improving service delivery to impoverished urban communities.

Community-managed water systems remain the dominant system in rural areas throughout the region, although there is limited evidence of shifts toward community-management-plus models. This concept accepts that the community management approach cannot provide the required quality and reliability of services. A strategy to improve the resilience of rural systems has been water safety planning, which emphasizes communities' involvement in managing water safety and security risks, and recognizes the role of governments and other actors in supporting communities to manage systems. Specific service delivery models are not discussed in the literature reviewed.

$\rightarrow$ Shifts toward community management models of rural WaSH need to include formal arrangements of support, technical and other, to be provided by governments or other actors, such as the private sector. Models suited to the context of Pacific SIDS are not yet identified in the literature reviewed.

4. Sociocultural Aspects of Water. The sociocultures of Pacific SIDS have important influences on water governance and management because of (i) coexisting formal and informal types of governance; (ii) the role of customary land tenure and attitudes toward land and water ownership; (iii) tensions between the demands of urbanizing societies and the traditional values of subsistence communities; (iv) the general attitude to change and external influences; $(v)$ the lack of trust in the capacity of institutions to provide core services. Highly participative approaches are recognized as the best ways to adequately consider and leverage sociocultural aspects. Specific issues and gaps include the following:

$\rightarrow$ Political stability and corruption: There is a need to better understand how to leverage traditional social systems, as obligations to one's community can challenge the equitable provision of services.

$\rightarrow$ Water resources and customary rights: There is a need to identify models involving landowners in water resource management, to overcome challenges related to customary land and water rights.

$\rightarrow$ Culture and water use behaviors: The understanding of users' strategies to manage multiple sources in case of water stress and the identification of behavior change strategies to manage demand seems necessary. There is a clear knowledge gap about willingness to pay for services, notably in informal areas. $\rightarrow$ Culture and sanitation: The effectiveness of emotionally based campaigns to promote sanitation and handwashing is not well-documented in Pacific SIDS. Research exploring ways to address social, cultural, and financing constraints, and evaluating the use of composting toilets, are not identified.

5. Unplanned Urbanization and Water. There is rapid growth in informal and squatter settlements in some parts of the Pacific. In Melanesia, the urban population size, urban security concerns, scale of squatter and informal settlements, access to land, political instability, ethnic tensions, and degradation of the urban environment suggest that urban challenges are more complex and diverse than those elsewhere in the Pacific. Informal settlements can hold high disease risks in large part because of poor sanitation facilities, poor water quality, poor land quality, and population densities that facilitate spread of infection. Government bodies may be reluctant to address issues of urban planning due to sociocultural issues and land ownership issues. Utilities often have no commercial incentive to extend services to informal settlements and sometimes face legal and technical barriers. When utilities or informal providers address a community's immediate water demands, complementary services such as drainage or wastewater treatment are usually not established. There is finally a need for projects integrated with 
urban planning and management at a city scale that promote an integrated urban water management framework.

$\rightarrow$ Policy makers should identify low-cost and innovative infrastructure, and service delivery models, and governance arrangements suited for informal communities. Urban governance that can raise revenue, ensure acceptable land use patterns, and facilitate both formal and informal decisions on land use and land development is also needed. This process requires more information on informal settlements and the nature of their functioning, because existing data are often very limited.

6. Service Delivery and Water Management Approaches for Small and Remote Populations: Related challenges include small economies of scale combined with high costs to acquire hardware and build or provide local capacity. Considering these aspects - as well as the sociocultural aspects of Pacific SIDScommunity-managed systems are the most appropriate model for rural and remote populations to achieve local water services and sustainable water resource management. A "community management plus" (Hutchings et al. 2016) system will be required, acknowledging the need for formal arrangements to provide ongoing technical and, in some cases, financial support for capital investments, rehabilitation, and major maintenance.

$\rightarrow$ There are knowledge gaps in understanding which community management plus models, utilizing what infrastructure and technologies, are effective and sustainable with Pacific SIDS, considering capacities, finance, supply chains, disaster resilience, and sociocultural factors. Other issues to explore include the feasibility of integrating water service delivery with other sectors (waste, energy, etc.).

Strategies to improve sector capacity need to explicitly target the capacity of small and remote communities. Accepting the inevitable emigration of newly skilled workers as a positive outcome for economic and human development would allow better planning for capacity development.

7. Water Data, Information, and Knowledge: Collection of information and knowledge relating to water resources and their use is often limited in Pacific Island countries. This can be largely attributed to a lack of resources to carry out analysis, ensure financing, and provide skilled workers. Information may also be unavailable due to a lack of data sharing and limited coordination between bodies responsible for water management. Some authors have noted a reliance on consultants (or alternatives, such as universities or training institutes) to build capacity to better share their knowledge and skills. Literature points to the importance of using local knowledge and participation in water resource assessments and plans, and in the establishment of (i) national legislated water resource monitoring standards; (ii) national and regional forums to foster sharing of information and lessons learned; and (iii) tools and methods to gather information and systematically assess and distribute it.

$\rightarrow$ Gaps in knowledge remain regarding the collation, and analysis of water resource assessments for specific contexts: outer islands, atolls, and rapidly urbanizing areas. Efforts are required to understand how to effectively collect and integrate information across geographic, disciplinary and cultural divides. There is a need to map waterborne disease prevalence and hotspots to help donors and international finance institutions focus WASH investments to areas of greatest need. 


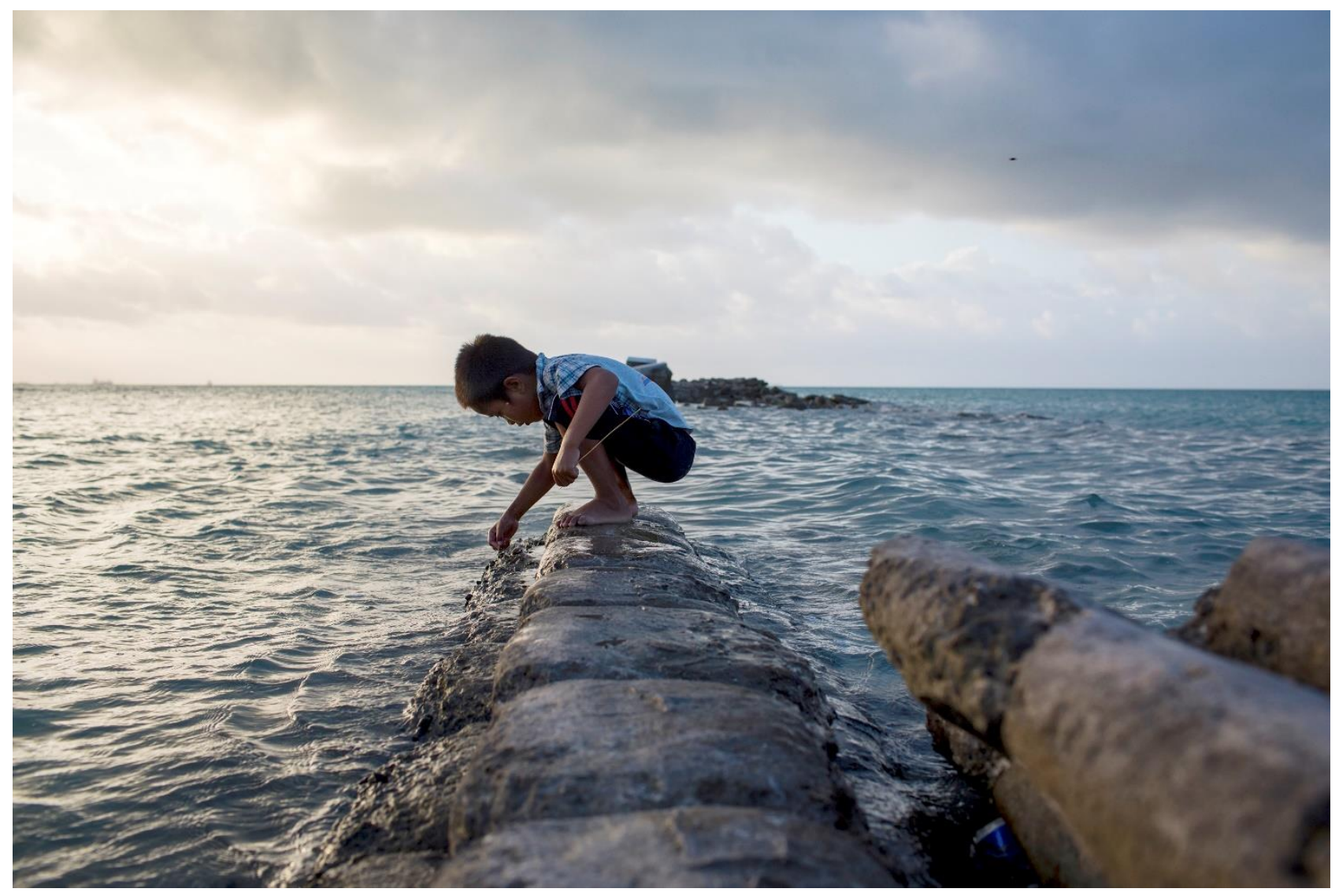

Boy playing on a flooded sea wall in Bikenibeu village, South Tarawa, Kiribati (Credit: World Bank)

\section{Environmental Variability and Climate Change}

\subsection{Nature of the Challenge}

Pacific Island countries experience environmental variability and change resulting from direct human impacts such as urbanization, population growth, and changing land use and land management (e.g., deforestation and mining), as well as environmental change attributed to natural climate variability and human-induced climate change. A study (Falkland 2011) summarizing risks to water security in 14 Pacific Island countries from climate change and nonclimate factors finds that the most significant water security risks up to 2030 include the following:

- Increasing water demands resulting from development and population growth

- Pollution of water resources resulting from development, population growth, and increasing urbanization, leading to major health impacts

- Salinization of fresh groundwater resulting from seawater intrusion due to overpumping

- Water leakage from many urban pipe distribution systems (in many situations greater than 50 percent) 
- Poor water governance and management, which are particularly evident during droughts

- Impacts from civil works or mining that make coastlines more vulnerable to erosion or expose shallow groundwater to contamination

Across Pacific SIDS, natural hydrogeology and environmental variability, and changes due to human and climate factors have a range of influences on sustainable water management. Water supply is expected to be an ongoing and increasing problem in the Pacific. Water supplies are threatened by naturally small reserves in some islands, climate change impacts, and population growth and rapid urbanization (resulting in increased demands for water and loss of water quality through contamination from a lack of sanitation and pollution controls) (South 2004). Drought management strategies and response plans will become increasingly important as water demand rises and rainfall variability increases in much of the region.

Greater monitoring and accessible information are needed regarding the extent and quality of water resources during drought periods. Policy makers also need a greater understanding of patterns of use of multiple water sources during different seasons, and at times of floods and droughts to address waterrelated health risks (Hadwen et al. 2016; White and Falkland 2010).

Climate change impacts mean that water supply, sanitation, and hygiene (WASH) are implemented in an increasingly unpredictable climate. Delivery challenges are exacerbated by extreme weather events, increasing climate variability (including droughts and floods), and changes over time to resources (such as groundwater recharge changes). WASH projects will need to be resilient to climate change impacts, and incorporate climate change into WASH policies, plans, and strategies (Hadwen et al. 2016).

Pollution poses a significant risk to water quality especially to shallow and small groundwater reserves of low islands; a lack of appropriate sanitation threatens water security and human health through contamination of water supplies (White and Falkland 2012b). Given the vulnerability of local water resources to pollution, water supply and sanitation (WSS) initiatives are critically important during disaster responses. WASH concepts will need to be integrated into disaster risk reduction (DRR) and climate change adaptation (CCA) initiatives (WHO 2015).

There is growing interest in understanding and supporting the use of multiple water sources rather than focusing on a single primary source of drinking water in settings where diverse sources exist; rural communities have traditionally managed water sources in this way (Hadwen et al. 2016). Application to urban settings is less obvious. New user-friendly tools and frameworks and capacity building will be required to collect and analyze data for risk management and protection of water resources in the face of climate change and to develop resilient WASH, CCA, and DRR plans (Hadwen et al. 2016).

\subsubsection{Effects of Urbanization and Population Growth on Pacific Island Countries}

Population growth and intensified urbanization in parts of the Pacific have led to increased water demands and pollution. Water quality degradation through inadequate sanitation and waste disposal is the most significant hazard to Pacific Island water resources (Scott et al. 2002), and a greater risk to water security than climate factors (White and Falkland 2012b). Inadequate sanitation and waste disposal practices, especially in crowded urban and peri-urban areas, have led to degradation of surface water and groundwater resources through biological and chemical pollution (Falkland 2011). Rural villages, with very basic water supply systems, can still be subject to polluted water supply streams, springs, and wells (Falkland 2011). 
On many islands, sanitation systems using pit toilets and septic tanks are a major source of biological contamination and health risks (White and Falkland 2010). Septic tanks are rarely maintained and often leak (Falkland 2011). Other sources of pollution can include fuel and oil spills and leaks; inappropriate and uncontrolled use of agricultural chemicals (fertilizers and toxic insecticides and pesticides) that affect surface water and groundwater; and industrial discharges and accidental spillages of toxic chemicals from mining sites into surface waters (e.g., from gold mining sites in Papua New Guinea) (Falkland 2011). There is often little government direction or regulation relating to water quality protection and pollution control (White and Falkland 2012b).

\subsubsection{Effects of Climate Change and Natural Disasters on Pacific Island Countries}

Geological hazards, such as volcanic eruptions and earthquakes, with resulting hazards of landslides and tsunamis, can occur in some Pacific Island countries. Tsunamis can cause temporary salinization of groundwater resources and wells through coastal inundation (Falkland 2011).

Climate change will likely result in most subregions of the Pacific becoming wetter and some drier, and that increased rainfall intensity and seasonal variability will be felt across the Pacific region (WHO 2015). El Niño-Southern Oscillation (ENSO) is a main driver of rainfall variability in the region, and is correlate with changes in surface and groundwater resources. El Niño episodes drive droughts in most of the Pacific Island countries and in East Timor, and cause heavy rainfall in the countries near the equator (Nauru and Kiribati) and in some islands of the Federated States of Micronesia and the Cook Islands. During La Niña episodes, major droughts are often experienced in Kiribati and Nauru and parts of the Federated States of Micronesia and the Cook Islands (Falkland 2011).

Pacific island countries are often unprepared for flood and droughts (WHO 2015). Common drought adaptation measures include balancing water use from rainwater, groundwater wells, and surface water supplies, and changing water use (see example in Hadwen 2016: 48). During droughts in areas such as Dili, East Timor, some households turn to contaminated water sources as a sole or supplementary water source (Falkland 2011).

Models suggest that tropical cyclones in the region will become more intense (Hadwen et al. 2015), with devastating consequences as shown on Figure 3.1. Cyclones often cause floods and hillside erosion resulting in downstream damage and sedimentation, and damage to water supply infrastructure (Falkland 2011). Flooding can lead to temporary contamination of drinking water supplies or adverse impacts on water quality.

Many Pacific Island countries have climate change and disaster risk management policy frameworks that seek consistency in water supply and management. Policy, programming, and technical challenges in implementing these policy frameworks exist in some countries; examples include limited coordination and

\section{Photo 3.1: Damaged homes in Fiji following Tropical Cyclone Winston}

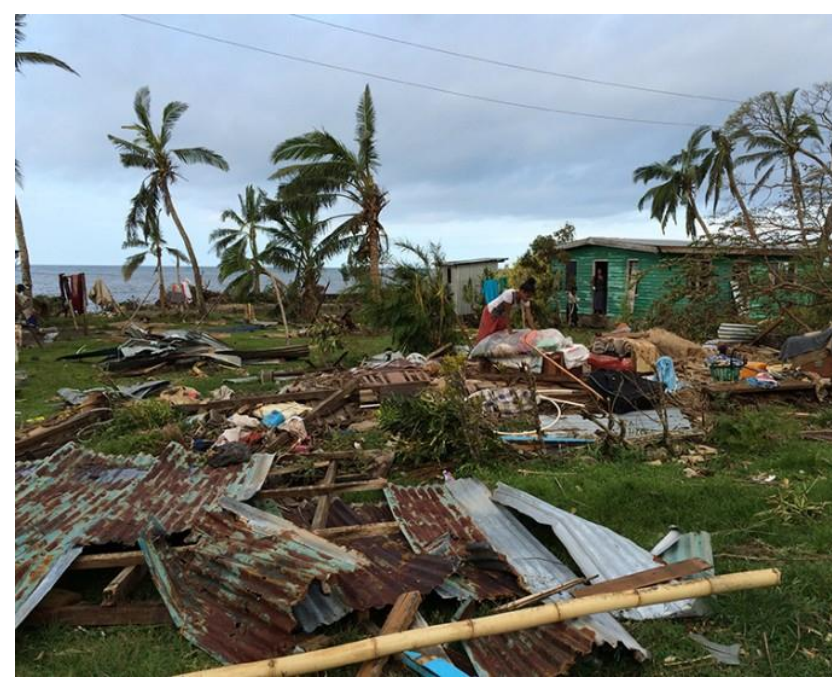

Credit: USAID/OFDA 
consistency across sectors and organizations, and limited information or capacity to analyze data and incorporate it into risk management frameworks and plans (Hadwen et al. 2016).

Processes and results of environmental variability and change are varied across and within Pacific SIDS. However, some experiences and challenges are shared across low islands (atolls and reef islands) and across high islands (volcanic, continental, and raised atolls). For instance, Nurse and McLean (2014) attribute water-related risks for each typology of island in Western Pacific: river flooding more likely to be an issue in large islands such as Papua New Guinea, flash floods in volcanic high islands such as Vanuatu, and storm surges, droughts, and vector diseases in atolls and limestone islands.

\subsubsection{Effects of Environmental Variability and Climate Change on Low Islands}

In atoll islands, limited land areas and high levels of soil hydraulic conductivity significantly reduce surface runoff and surface storage. Fresh groundwater lenses sitting over seawater are the major source of fresh water for many communities on atolls. This groundwater is vulnerable to droughts associated with ENSO, salinization due to storm surges, sea level rise, and human activities (contamination and groundwater salinization as a result of overextraction) (Falkland 2011).

In low coral atolls the water table is close to the soil surface, resulting in rapid movement of superficial contaminants into groundwater (White et al. 2007a). Traditional practices include the location of potentially polluting activities in groundwater discharge zones to protect groundwater quality. However, as populations grow and urbanization ensues in many atoll islands, these practices become more difficult to observe (White et al. 2007a).

\section{Box 3.1 Non-Pacific SIDS-Climate Change Impacts of Precipitation and Sea Level Rise in the Maldives}

In the Maldives, the effect of sea level rise on the freshwater lens was quantified using general circulation models with estimates of shoreline recession and associated decreases in island width. If rainfall is solely considered, available groundwater is projected to increase due to a projected increase in precipitation during 2031-50 compared to 2011-30. However, including the impact of sea level rise indicates an overall decrease in lens thickness, with drastic decreases (60 percent to 100 percent) projected for small islands (200 meters) and moderate decreases (12 percent to 14 percent) expected for 400-meter islands, which accommodate one-third of the population of the Maldives.

Sources: Deng and Bailey 2017.

Water problems for atoll islands and cays are critical, especially in urban and peri-urban low coral atoll communities in which land is limited. Here, limited land supply constrains the storage of freshwater during dry periods. Additionally, managing demand for water and waste disposal is challenging due to increasing populations (White et al. 2007a). In groundwater-dependent atolls, subsistence crops "compete with humans for fresh water" (White et al. 2007a).

Growing urban communities may pollute groundwater with human and animal wastes (particularly those of pigs), resulting in endemic waterborne diseases (White et al. 2007a). In small, low-lying coral islands where the soils are highly permeable and groundwater is extracted from nearby wells, inappropriate sanitation systems such as pit toilets and flush toilets with septic tanks have often been installed. These systems are prone to causing groundwater contamination (Falkland 2011). Shallow household wells have been contaminated, including fecal contamination, creating considerable health problems (ADB 2012a). 
Accelerating rates of sea level rise are very likely to yield severe risks for low-lying coastal areas and atoll islands. Extreme sea level events such as swell waves and storm surges present severe flood and erosion risks, while seawater overwash threatens to degrade fresh groundwater resources. Overtopping by seawater is a risk for islands with low-lying aquifers, especially during extreme events such as hurricanes. Freshwater lenses recover from overtopping over time, but remedial actions such as draining trenches can help shorten recovery time. One of the mechanisms of aquifer contamination following a storm surge is from open wells or trenches that provide direct access to the water table and collect the salt water during inundation. (Holding and Allen 2015)

Risk profiles of small islands in relation to these climate-related drivers varies according to their natural environmental variability, physical and human aspects. Consequently, the transferability of climate adaptation initiatives across islands is not always appropriate (Nurse and McLean 2014).

\subsubsection{Effects of Environmental Variability and Change on High Islands}

In high islands, catchments with steep slopes enable water and pollutants to move quickly to downstream areas and the coastal zone. Extensive deforestation on slopes leads to erosion, increases sedimentation and turbidity of surface waters and coastal environments, and exacerbates flooding problems. Extensive land clearing has resulted in impacts on spring flows, stream flows and water supply infrastructure in East Timor, the Solomon Islands, and Upolu, Samoa (Falkland 2011). Cyclones and heavy rainfall events can damage the water supply infrastructure or temporary contaminate drinking water supplies when flood waters containing debris and biological contaminants such as human feces inundate wells and surface water bodies (Hadwen et al. 2016).

Volcanic activity in some high islands can present challenges to water supplies including contamination from ash and damage from volcanic blast (Falkland 2011). Earthquakes can cause cracking of water supply pipes and rainwater tanks, resulting in short-term emergency water needs. (Falkland 2011)

\subsection{Ways Forward}

UNICEF Pacific began its WASH resilience program in 2015 with the aim of allowing beneficiaries to not only receive improved WASH services, but also gain an ability to reduce exposure to future difficulties, be able to adapt to changing difficulties, and have an ability to minimize difficulties when they occur. The approach recognized that achieving resilience requires working with risk at multiple levels:

- Strategic and national level: to ensure that resources are applied in areas where risks are high

- Programmatic level: to ensure that the correct projects are being undertaken that directly contribute toward national, provincial, and strategic goals

- Operational level: to ensure achievement of sustainable improvements in WASH on the ground

Risk assessment is used to inform the prioritization and design of funding proposals, investments, and activities of WASH programs in relation to sustainability and a changing climate. As one key outcome, communities will improve the safety, security, and sustainability of their drinking water and sanitation systems to cope with current climate change and disaster risk and adapt to future climate changes. To that end, a comprehensive model for WASH resilience will be developed and used. 
These goals will be achieved by undertaking a policy review of WASH resilience in disaster risk reduction (DRR) and climate change adaptation (CCA) instruments; compilation of lessons learned; establishing a model of knowledge exchange; and developing a comprehensive model for WASH resilience.

\subsubsection{Technological and Infrastructural Approaches}

Some approaches include using seawater for flushing piped sewerage systems to conserve limited freshwater supplies, as in Majuro, the Marshall Islands, and Tarawa, Kiribati (White and Falkland 2010). Technical measures to control and limit pollution sources may include improved septic tanks and using simple effluent and disposal systems (White and Falkland 2010). Using composting toilets to protect freshwater lenses and conserve water has been tested in Kiribati, Tonga, and Tuvalu). Note that social acceptance of compost toilets has varied among island populations (White and Falkland 2010).

Some areas have benefited from using infiltration galleries (also known as "horizontal wells" or "skimming wells") for pumping groundwater in small coral islands and coastal areas of smaller volcanic islands, instead of using conventional boreholes. Because infiltration galleries pump over a wider area of the freshwater lens than conventional processes, they avoid problems of saline intrusion into groundwater. Infiltration galleries have been established in Tarawa and Kiritimati, Kiribati; Kwajalein in the Marshall Islands, and Lifuka, Tonga (Falkland 2011). Another option is metering and monitoring of pumps in areas with potential risks of saltwater intrusion into fresh groundwater due to overextraction. Metering and monitoring may help to ensure that groundwater pumping rates do not exceed sustainable yield (Falkland 2002; White and Falkland 2010).

There are many successful rainwater harvesting projects for households and community buildings in Pacific Island countries (Falkland 2011; Hadwen et al. 2016). Rainwater harvesting schemes provide potable water, except in severe droughts. They include rainwater tanks as well as rainwater harvested from specially prepared catchment areas, such as a paved airport runway in Majuro, the Marshall Islands (Scott et al. 2002). Another example is public housing designs for the main center of Fogafale on Funafuti atoll, which incorporates separate underground cisterns for private household use and for communal use during drought (Falkland 2002). Where rainwater storage is pursued to increase resilience to drought, maintenance requirements must be considered (Hadwen et al. 2016), as well as social structures and cultural issues (MacDonald et al. 2017). Hadwen et al. (2016) concludes that rainwater harvesting infrastructure offers greater water security if owned and managed by rural householders, rather than by communal or community systems, which rely on a high level of social cohesion. Finally, design standards for water supply and sanitation systems (adopted in some Pacific Island countries) could be further developed with guidance or tools to support climate resilient design and management (Hadwen et al. 2016).

Box 3.2 Non-Pacific SIDS-Barriers and Incentives to Increased Resilience through Rainwater Harvesting in the Caribbean

Rainwater harvesting is a simple, low-cost water supply technology that is generally easy to install and maintain. In spite of these considerations, the practice has been declining in the Caribbean as communities have become better serviced by central municipal systems. However, it continues to be a main source of water supply in many of the drier islands of the Caribbean, notably St. Vincent and the Grenadines, the Leeward islands, both the U.S. and British Virgin Islands, and the Bahamas.

The emerging trend in some of these islands is to move away from traditional rainwater harvesting methods in favor of alternative technologies such as desalination and deep well abstraction. In Antigua and Barbuda, rainwater harvesting has been built into infrastructure and development policy: all dwellings must be built with 
facilities to store at least three to four days of water requirements based on house size. In Barbados, rainwater harvesting was traditionally practiced but has been on the decline. However, an amendment to the Town and Country Planning Development Order, 1972 requires all new residences in Barbados to make provision for rainwater storage. A tax rebate incentive is applicable.

In the British Virgin Islands, rainwater harvesting is mandatory. The government has issued loans and subsidies to construct cisterns and, more recently, has distributed plastic tanks to farmers. In Dominica, which is less water scarce, rainwater harvesting is not significant although it is assumed that some households in remote areas that are not connected to the distribution network practice it. In Grenada, rainwater harvesting is practiced to a small degree on the main island, but more so by households in communities particularly in the south of the island where there are serious supply problems. By contrast, rainwater harvesting is of paramount importance in Carriacou and Petit Martinique where communal and private systems supply almost 100 percent of demand. There are no specific policies or legislative provisions to support rainwater harvesting in Grenada.

In Jamaica, rainwater harvesting systems are common in areas with no reliable public water supply and in areas lacking access to rivers, springs, or well water. It is estimated that more than 100,000 Jamaicans depend on rainwater harvesting as the primary source of water. The Turks and Caicos Islands rely almost exclusively on rainwater harvesting, and all developments must include storage capacity at a ratio of 400 liters per square meters of roof area. In addition, there are a number of government-built, public rainfall catchment systems. It is a legal requirement in the U.S. Virgin Islands that all buildings must have rainwater harvesting systems installed with 400 liters of storage for each square meter of roof area. Cistern construction is further regulated by the Virgin Islands building code to ensure the structural integrity of these cisterns, which usually form an integral part of building foundations. Barriers to rainwater harvesting practices include concerns for mosquito breeding, the cost of guttering and storage, and that stored rainwater is often not handled to minimize contamination (in Saint Lucia 80 percent of stored rainwater tested was not safe for consumption according to World Health Organization [WHO] standards).

Sources: GEF/ IW:LEARN 2014.

The success rate of desalination has been poor in Pacific Island countries because the equipment is expensive to operate and maintain. However, in places such as Majuro atoll, where existing freshwater resources (surface water, groundwater, and rainwater catchments and storage) are inadequate to cope with demand during droughts, desalination units have been installed and are considered more favorable (Falkland 2011).

One interesting proposal for infrastructure to address water supply and drought management in Kiribati is for a complete island that would be reclaimed by dredging an area of a lagoon in North Tarawa. The island would be dedicated to supplying water and would be on government-owned land, therefore bypassing needs to rent or negotiate with land owners. However, the proposal would be expensive, may have adverse environmental impacts, and would require time to build up a fresh groundwater lens. The time required would be too long for the proposal to meet South Tarawa's immediate water needs, but longer term could contribute to meeting water supply needs. The addition of a major photovoltaic array for renewable energy generation has been raised so that the value of land use on the proposed constructed island would be sufficiently high to justify the expenditure to build it (White 2010).

Monitoring has helped assess water resources over time and inform robust adaptive and timely measures to protect them. This is especially the case for areas dependent on groundwater lenses that are under increasing demands. In these areas, ongoing monitoring and analysis is needed to determine suitable groundwater development to minimize salinity and promote effective management of groundwater source areas (White and Falkland 2010). Public participation, training, and capacity building for water resource assessments may assist in the development of drought management strategies. Water resource 
assessments can include community knowledge as a valuable component alongside technical and scientific approaches (Falkland 2002).

A severe drought throughout Kiribati in 1998 and 1999 highlighted the need for appropriate quantitative measures of the severity of drought or a drought index that could take into account different sources of water for domestic supplies (GEF 2007). The South Tarawa Drought Response plan uses a drought index based on monthly rainfall to determine different levels of alert and corresponding actions required (Falkland 2011; White et al. 1999).

\section{Box 3.3 Financing Rainwater Harvesting in Tarawa, Kiribati}

Through the Sanitation, Public Health and Environment Improvement Project (SAPHE) project, delivered with the Asian Development Bank (ADB), a revolving fund loans scheme was established for government employees to install household rainwater harvesting or sanitation facilities. Approximately 710 loans were given for rainwater harvesting and storage equipment from 2002 to 2004 to install 6,000 liter polythene tanks and associated gutters and downpipes. To ensure the repayment of loans, they were given only to people with regular incomes and recovering regular repayments from fortnightly salaries (GEF 2007).

The project was considered a success because of the direct benefits to households, and because of its demonstration of "self-help" with householders taking responsibility for improvement to their own water security. This type of project has implications for other urban areas facing challenges associated with droughts in which householders have access to a consistent income and can repay a loan (Falkland 2011). Under the pilot implementation phase of the Kiribati Adaptation Plan (KAPII) this scheme was to be trialed in rural areas and outer islands, as shown on Photo 3.2:

Photo 3.2: KAP-III rainwater harvesting scheme in North Tarawa, Kiribati

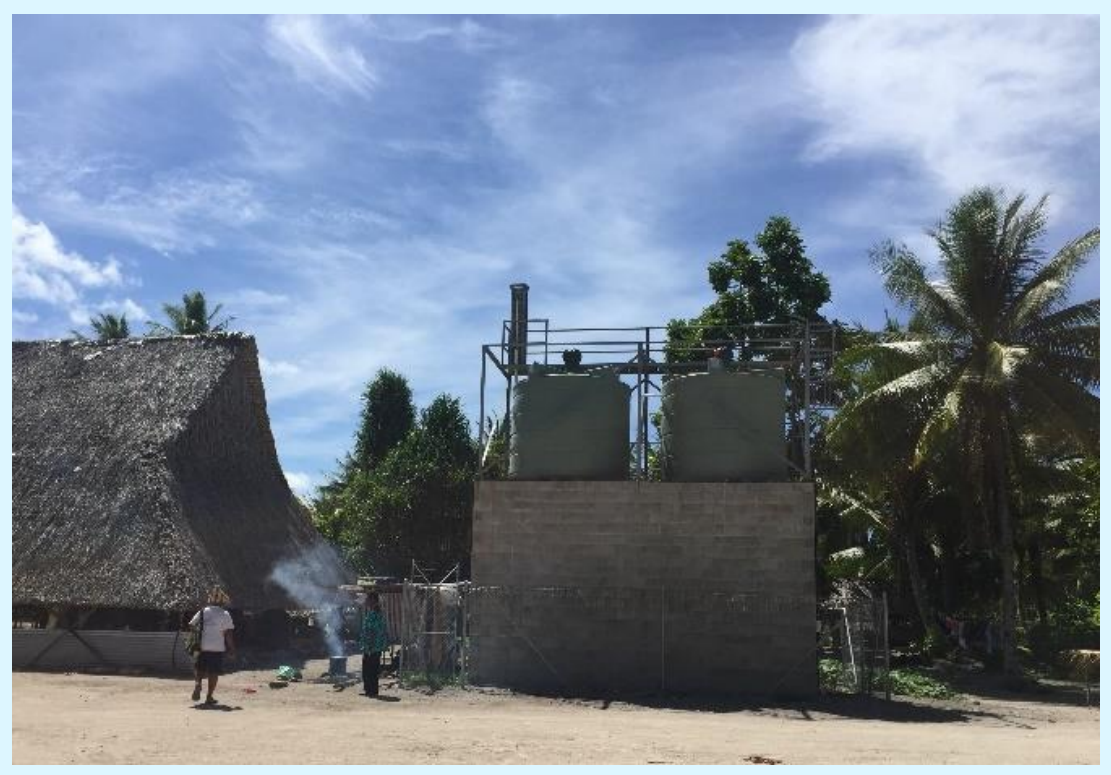

Source: Van Anh Vu Hong/World Bank

Device-based surveys are used to better understand patterns of water use across different seasons and during flood and drought events in flood-prone catchments in the Solomon Islands, and drought-prone atolls in the Marshall Islands (Hadwen et al. 2016). The device-based survey assists the rapid collection and immediate access to complex multiple water source data from communities, with the potential to 
assist policy makers to identify risks for disaster risk management and climate change adaptation in real time (Hadwen et al. 2016).

\subsubsection{Policy and Strategic Planning}

Public policies, goals, and implemented policies and plans are needed to address threats to water quality and quantity stemming from environmental change and natural hazards. There is growing interest in integrated system approaches that seek to incorporate connected aspects of catchment and water management, such as climate change impacts; current WASH systems; potential for maladaptation to climate change impacts and adaptation opportunities; collective impacts of interventions; technological aspects; and input (including data and information) from a range of stakeholders (Hadwen et al. 2016).

Implementation of water quality protection policies needs long-term support from regional organizations, aid and donor agencies, and Pacific Island countries themselves (White and Falkland 2012a). Hajkowicz and Okotai (2006) recommend investigation into alternative policy instruments for achieving catchment management improvements while meeting other local needs. Touted policy instruments include tax subsidies, competitive tendering for watershed improvement contracts, incentive payments, awareness schemes, tradable permits, and regulations (Hajkowicz and Okotai 2006).

Policy and strategic planning responses to extreme weather events and other disasters can be tied to, or include aspects of, disaster risk reduction (DRR), climate change adaptation (CCA), WASH, and integrated water resource management (IWRM). Drought response is an essential component of CCA strategies, DRR strategies, and IWRM in all Pacific Island countries, because they are all affected by drought to some extent (Falkland 2011). There is a move away from disaster response paradigms toward approaches of hazard assessment and risk management, with a key role for the WSS sector (WHO 2015). These approaches are inclusive of disaster response, recovery, preparation, and prevention, and use postemergency and disaster assessments to inform future development and planning (UNICEF 2017a). In countries with limited financial and human resources, a risk-based management approach can help to identify the greatest risks and address them first (UNICEF 2017a). Several Pacific Island countries have "reasonably well-defined climate change and disaster risk management policy frameworks" (UNICEF 2017a).

There are growing efforts to integrate DRR, disaster risk management, and CCA policy frameworks and institutional arrangements, which have traditionally been distinct, to improve coordination and make efficient use of limited human and financial resources for monitoring, assessment, and management of disaster and climate risks (e.g., in the Marshall Islands, Vanuatu, Fiji, and the Solomon Islands) (Hadwen et al. 2016). Care is needed to avoid institutional overlaps (Nurse and McLean 2014). There are also growing efforts to ensure WSS is reflected in national CCA and DRR plans (WHO 2015), and that WASH initiatives seek to be climate resilient and adopt an IWRM approach (Hadwen et al. 2016). Tonga has a joint CCA and DRM plan that includes specific water sector adaptation strategies and a strong level of policy harmonization across issues of WASH, CCA, and DRR (UNICEF 2017a).

Capacity building for CCA and DRM that also fosters cross-sectoral partnership and coordination has been promoted (Hadwen et al. 2016). WASH can be a tangible entry point to enhance adaptive capacity to disasters and climate change as communities recognize the importance of WASH (UNICEF 2017a).

A recent review of WASH plans and policies adapted in Pacific Island countries finds that they often lack guidance or information to support climate resilient management and design. There have been efforts to 
engage governments and communities to increase their capacity to manage and assess risks to ensure WASH projects are resilient to natural disasters and climate change, and to develop risk-based CCA and DRR plans. Such efforts should seek consistency in tools and approaches used across local groups and reduce dependency on external actors. (Hadwen et al. 2016)

Drought management strategies and water conservation help address climate change threats to water security in the region. Efforts to improve drought management include improvements to water supply infrastructure, such as rainwater capture and storage or pipe leakage reduction, or improvements to community and water sector knowledge of available water resources, their quality, and demand (White et al. 2007a).

The Tarawa Water Master Plan, Kiribati, is an example of the application of data to strategic planning to mitigate the impact of drought. The plan considers rates of current groundwater extraction and assesses future extraction levels, taking into consideration population growth, anticipated climate change impacts (such as saltwater intrusion into fresh groundwater due to sea level rise), and other nonclimatic impacts (e.g., contamination of groundwater). It then identifies ways to improve monitoring of water resources and use of that data to inform water planning and decision making, including climate adaptation plans. Finally, it assesses alternative responses to addressing water security challenges. (White 2010)
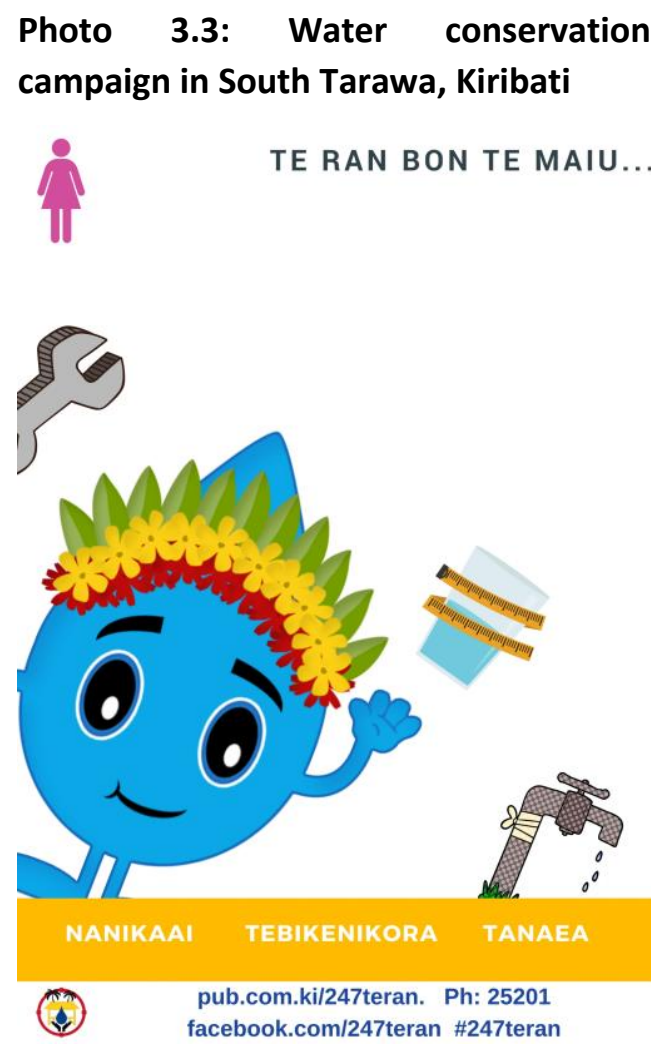

Source: KAP-III project

\subsubsection{Increased Resilience of Community Water Systems}

Communities may be reluctant to plan for disasters when they are unable to address their daily needs. Therefore, incorporating resilience and disaster planning into community WASH planning approaches should be prioritized. Approaches such as that in UNICEF (2017b) builds on the WHO's risk-based WASH safety planning approach and considers variability and climate change to water quantity as well as risks to water quality.

In Tonga, participatory research on ecological sanitation has addressed problems of groundwater pollution from septic tanks, pit latrines, and domestic animals. A village in the Ha'apai group of islands trialed a dry composting sanitation system, considered beneficial because it did not discharge effluent into the lens and did not use limited water resources for flushing. The participatory approach meant that community awareness of groundwater pollution from sanitation facilities increased and improved research outputs. A review of the project several years later finds that community members still consider the composting toilets to be beneficial and suitable. However, maintenance of some toilets was inadequate. This project is considered a good example of sanitation research that actively engages the community and thus gained community support and uptake (Crennan 2005). 
The Solomon Islands Water Sector Adaptation Program (SIWSAP 2014-19) will address water sector vulnerabilities as identified in the National Adaptation Program of Actions (NAPA) for Climate Change adaptation. The project aims to improve the resilience of community water resources to the impacts of climate change to improve health, sanitation, and quality of life, and sustain livelihoods in targeted vulnerable areas. Six pilot sites were identified based on the limited water supply in the area. Championed by the Solomon Islands government through the Ministry of Mines, Energy and Rural Electrification (MMERE) Water Resources Division (WRD), in partnership with the Ministry of Environment, Climate Change, Disaster Management and Meteorology (MECDM) and other line ministries, SIWSAP activities are designed to ensure access to safe and affordable drinking water and increase reliability and quality of water supplies in targeted areas. Longer-term project measures include working to integrate climate resilient water management in policy and development frameworks; encourage investments in costeffective and adaptive water management technologies; and improve governance and knowledge management for climate change adaptation in the water sector at the local and national levels.

SIWSAP utilizes a participatory approach to combine locally held knowledge and internationally verified climate science in an effort to achieve greater use of risk data at the operational level. There is a community engagement process to water safety planning, but with a stronger emphasis on climate change observations and predictions. During the community consultations, the participants are encouraged to describe any changes to their climate and environment observed over the last 20 years. In addition, the program staff produce a location-specific report on future climate change predictions that can be used by community members in their risk reduction management plans. The combination of locally held knowledge and internationally verified climate science is a strength of the approach (UNICEF 2016a).

\subsubsection{Demand Management}

Managing the demand for water and water use efficiencies is an important component of managing limited water resources. Because of its strong link to changing human behaviors, this topic is addressed in chapter 6.

\section{Box 3.4 Kiribati Climate Adaptation Programs}

The last 15 years have seen significant investment into climate change adaptation in Kiribati, with water being a major consideration. The evolution of investments perhaps illustrates at least one pathway to achieving countrywide engagement and leadership on sustainable water management. Coordination among actors and operationalization of well-developed adaptation strategies appear to be key considerations.

In 2003, the Kiribati government started the Kiribati Adaptation Program (KAP), an example of a program utilizing many of the ways forward described previously. This program has the key goal of reducing Kiribati's vulnerability to climate change, climate variability and sea level rise, through three phases (GEF 2007):

- Preparation phase (2003-05) to incorporate adaptation into national economic planning, prepare a national adaptation program of action (NAPA), and design an intermediate pilot implementation phase.

- Pilot Implementation phase (2006-08) to develop and demonstrate the systematic diagnosis of climaterelated problems and the design of cost-effective adaptation measures, while continuing the integration of awareness and responsiveness into economic and operational planning. This will be achieved through continued consultation and awareness raising; consolidation of the mainstreaming of adaptation into national economic planning; and implementation of pilot adaptation measures to address pressing adaptation issues while building capacity in key government ministries, local government, and communities. 
- Expansion (2009-15) to gradually scale up the investments piloted under phase II to cover all major islands and vulnerable sectors of Kiribati.

KAP was financed through grants through the World Bank from the Australian government; the Global Environment Facility (GEF) Least Developed Country Fund (LDCF); Japan Policy and Human Resources Development (PHRD); the Global Facility for Disaster Reduction and Recovery (GFDRR); and in-kind contribution from the Kiribati government. Toward the end of KAP, the Kiribati Joint Implementation Plan for Climate Change and Disaster Risk Management (KJIP) 2014-23 was developed. This plan intends to integrate climate change and disaster risk management considerations into existing implementation, financing, and monitoring functions. It is also designed to strengthen coordination and communication among ministries as well as civil society and development partners, including the Kiribati National Expert Group on Climate Change and Disaster Risk Management (KNEG) and an accompanying secretariat, both operating under the Development Coordinating Committee.

The KJIP will be monitored through the Kiribati Development Plan Monitoring and Evaluation Framework (2012) in compliance with the Monitoring and Evaluation Policy. At the ministerial level, KJIP strategies will be monitored through the annual Ministry Strategic Plans, which will incorporate relevant KJIP actions and outcome indicators. The KJIP is understood to be a living document and as such the KNEG can adjust it to meet emerging needs, to be approved by the Development Coordinating Committee.

More recently, the United Nations Development Programme (UNDP) reports it is working with Kiribati to develop a project proposal for a new US\$9 million grant proposal for the Global Environment Facility Least Developed Countries Fund. The proposed Enhancing "Whole of Islands" Approach to Strengthen Community Resilience to Climate and Disaster Risks in Kiribati project will include US\$45 million in co-financing. The project aims to strengthen the capacity of government institutions to support the operationalization of the KJIP, enhance capacity of island administrations to plan for and monitor climate change adaptation processes in a "whole of islands" approach, and enhance community capacities to adapt to climate-induced risks to food and water security and community assets.

\subsubsection{Catchment Management and IWRM}

Using an IWRM approach, interventions might be identified that can improve the availability and quality of water resources, improve resilience to climate change, address catchment pollution issues, and as a result reduce risks within WASH systems. For example, actions taken to protect surface water quality (such as riparian restoration and improved land management) can also reduce health risks associated with poor water quality. This approach requires consideration of relationships between all activities in a catchment before implementing actions (Hadwen et al. 2015). For many areas, this may include investigation into the pathways for pollutants entering surface waters or drinking water supplies, and identification of sites where improved land management or other practices may yield the greatest impact on water quality (Hajkowicz and Okotai 2006). In some islands that are dependent on shallow and limited groundwater resources, clearing of deep-rooted vegetation (in appropriate locations) to reduce evapotranspiration losses from groundwater has been successful, especially where it is carried out with domestic rainwater harvesting (White and Falkland 2010).

\subsubsection{Land Use Planning and Management}

In some coral atoll Pacific Island countries, land use planning to protect groundwater recharge zones and regulation of land use in reserves have been used to manage freshwater lens areas. However, this strategy can be difficult if there are pressures on limited land areas, and when this does not correspond with customary land ownership and management. Keeping human settlements away from freshwater lenses used for public water supply may work only for a limited time if contamination sources are not dealt with 
(White and Falkland 2010). The effectiveness of regulations to control behavior and settlement on water reserves in Kiribati has been questioned, and alternative approaches to urban management that focus on community partnerships have been suggested instead (GEF 2007).

\subsection{Gaps in Knowledge}

Social and economic costs of climate change impacts and adaptation options on Pacific islands are rarely known. This is in part a result of limited climate and environmental monitoring frameworks (Nurse and McLean 2014). There are limited data from WASH practitioners for risk management that is accessible and user friendly. This may also be an issue of capacity, in that practitioners might not have capacity to integrate their information to a risk management context (UNICEF 2017a).

Accessible information is needed about the extent and quality of water resources so that Pacific Island countries are better able to manage them (White and Falkland 2010). Further information on local atoll groundwater systems is needed to minimize uncertainties in water security issues (Werner et al. 2017). Information is also needed on the hydrogeologic properties of atolls and anthropogenic influences to plan for the occurrence and recovery of disaster events (Bailey and Jenson 2013).

Designs are needed for individual wastewater treatment systems that are suited to different Pacific SIDS contexts, including environmental, sociocultural and economic. Identification of water and waste technologies for disaster recovery and rehabilitation-that might also contribute to longer-term WASH resilience in different environmental, sociocultural and economic contexts-would contribute to greater benefits from disaster recovery and rehabilitation investments. Capacity building is needed for practitioners to make appropriate technology selections in the context of local environmental, social, and economic factors. Convard et al. (2005) suggest research should be carried out on wastewater reuse for Pacific Island countries with wastewater treatment systems (such as Fiji, the Federated States of Micronesia, Vanuatu, and the Solomon Islands).

Gaps in knowledge remain regarding rural and peri-urban household water management practices, how they may vary by season, and how they can impact vulnerability to climate change (Hadwen et al. 2016). For example, there is limited information on the proportion of rainwater used for domestic consumption, and how this relates with use and supply of other water sources (White and Falkland 2010). Finally, systematic monitoring is required across urban water supply systems to identify leaks and deteriorating water quality, and to inform water demand management and drought management strategies. 


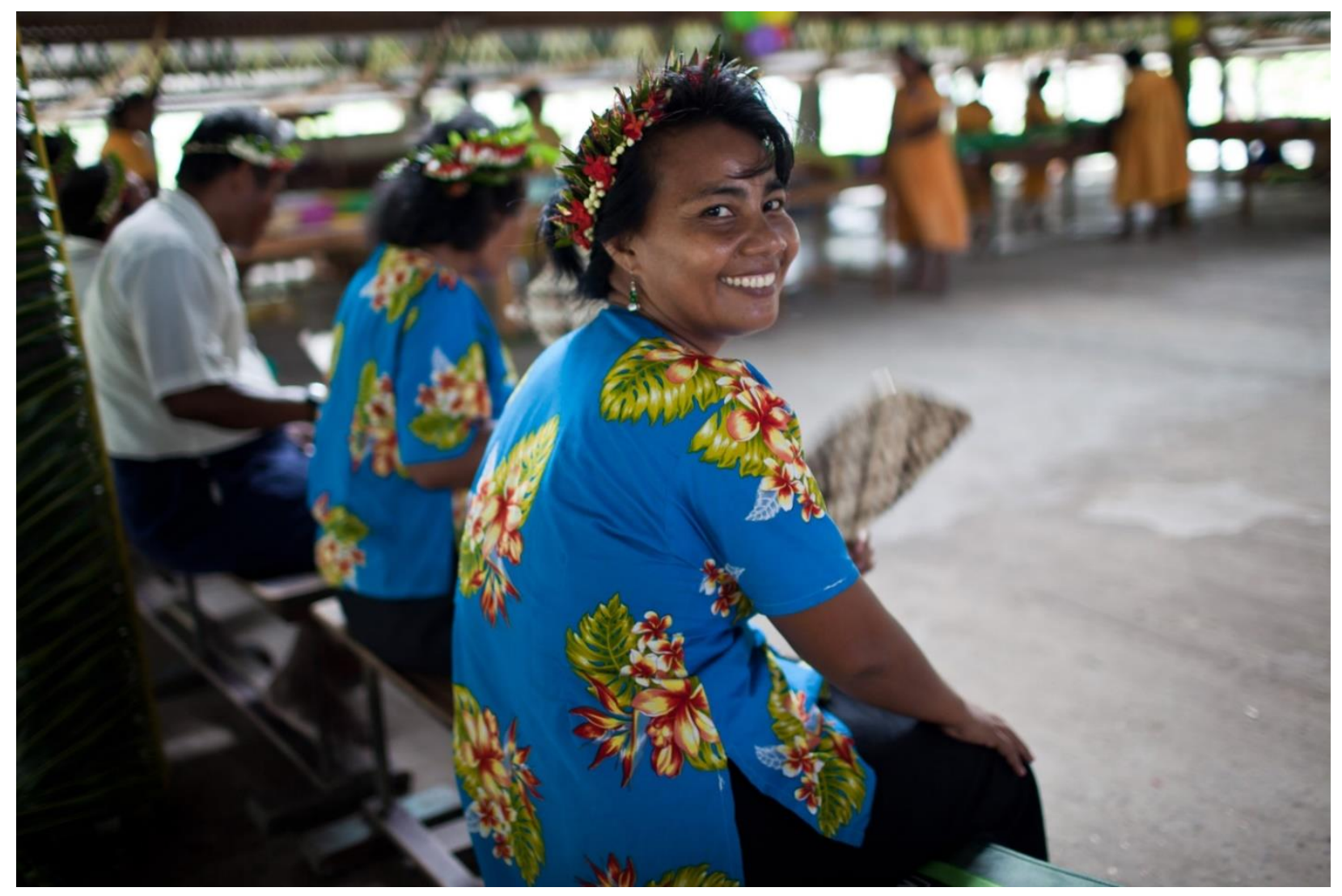

(Credit: World Bank)

\section{Water Sector Governance, Coordination, Integration, and Capacity}

\subsection{Nature of the Challenge}

\subsubsection{Need for Coordinated and Integrated Sectors}

Intersectoral coordination and integrated approaches to water policies are needed because water is a resource that affects and is impacted by many different environments, human activities, and sectors. Integrated approaches maximize economic and social welfare equitably-without compromising the sustainability of vital ecosystems - through coordinated development and management of water, land, and related resources. Failures in water management are often associated with a lack of coordination and communication across sectors and different levels of the governance system, resulting in fragmented management and misunderstandings (Keen 2002).

Hill (2002) summarizes the Pacific consultations on water management and observes that the water sector involves a complex relationship of environmental, social, economic, and cultural activities; rights, including from the perspective of water as a human right and the United Nations (UN) Convention on the 
Rights of the Child; and responsibilities. However, it has not been brought under a single focus so that the beneficial gains from integrated management-efficiencies of planning and operation, conservation of water and aquatic environments, equitable access arrangements, and financial savings in capital infrastructure works-can be realized.

White et al. (2006 and 2007a) recommend that water governance in Pacific Island countries generally, needs reform because (i) national water (and sanitation) policies and regulations are limited in scope or absent altogether; (ii) the limitations and lack of policies and regulations cause government agencies to lack clearly defined roles and responsibilities, apart from those inherited from older legislation and past administrations; (iii) silo water organizations exist, and several organizations overlap in their roles and responsibilities; and (iv) there is lack of coordination between donors and international organizations. In addition, in many cultures, land tenure has customary rights including ownership of groundwater (White et al. 2006).

The issue of water ownership is a significant "stumbling block to the adoption of water policy in many Pacific Small Islands," and the idea of vesting water ownership in the state conflicts with traditional practices (White et al. 2006). Hill (2002) also confirms the need to incorporate customary structures and institutions where practicable, and to actively involve the community in developing the driving vision, objectives, and action plans. Achieving a vision of sustainable water governance and management will almost certainly require the realignment of personal and collective rights and responsibilities (Hill 2002), this highlighting the critical requirements for community involvement in water governance in Pacific Island countries.

Integrated approaches to water management means holistic management of water across the natural and anthropogenic water cycles, and between water systems and other systems such as health, agriculture, and industry, to ensure full consideration of social, environmental, and economic aspects. Hill (2002) notes that a great deal of attention was previously given to establishing water utilities and other models of service providers, which was justified in terms of getting better and more efficient water supply networks in place. However, other parts of the water sector-wastewater, storm water, catchment and river control, aquatic ecosystems, and flood hazard mitigation-have progressed less well. Applying system-based approaches to water management is a recognized approach to improving integration and supporting coordination, but the review has found limited evidence of system analyses of country water systems.

An analysis of IWRM in Fiji (Keen 2003) identifies several requirements to improve integration and coordination including (i) public-private partnerships (PPPs) for catchment management and urban water management; (ii) better institutional communication and collaboration; and (iii) development of waterland management policies compatible with development goals and sociocultural norms, particularly those concerning resource tenure. An analysis of governance weaknesses in Kiribati (White et al. 2006) identifies that the impediments to good governance in Kiribati are the absence of (i) national WSS policy, water legislation, and approved strategic water plans; (ii) a whole-of-government approach; and (iii) community participation at the national and local village levels. These problems are compounded by organizational overlaps and conflicts and limited capacity and resources. The absence of national policy and plans makes the country vulnerable to the agendas of external donor and loan organizations (White et al. 2006). Dworsky's (2002) summary of constraints to sustainable water and wastewater management by Pacific 
region utilities identifies that multiple government agencies are involved in an uncoordinated and fragmented way, resulting in poor regulatory or policy links between the various sectors.

A lack of integration and coordination among government agencies has led to inequalities and gaps in service delivery for some subpopulations, in particular informal (unrecognized) settlements. With increasing urbanization within culturally complex Pacific Island countries, informal settlements in urban or peri-urban locations are rapidly growing (see chapter 7). Shields et al. (2017) conducted an analysis in 2015 of national water supply, sanitation, and hygiene (WASH) policies in Vanuatu, Papua New Guinea, the Solomon Islands, and Fiji. They identify that none of the policies include informal settlements; the tendency is toward policies dedicated to rural populations with a mandate for water utilities to provide services to formal urban populations, which means that informal urban and peri-urban populations are not addressed by national policies or programs. Fiji has since prepared a draft national WASH policy that explicitly considers all people.

Achieving national coordination among water-related agencies and integrated approaches to water management is further complicated by the small, dispersed populations of Pacific Island countries, impeding efforts to build sufficient local capacity and maintain functioning relationships among dispersed stakeholders. Dworsky (2002) states that while weaknesses in both public and private sector capacity are a key problem for most low-income countries (LICS), smallness adds a further dimension to the challenge. This problem is compounded in Pacific Island countries where internal distances are large and populations are scattered. In the public sector, small states face diseconomies of small size in providing public services and in carrying out the business of government, and tend to have relatively larger public sectors than other LICs. In the context of globalization, small states find they do not have sufficient institutional capacity to participate fully in international finance and trade negotiations - the outcomes of which can profoundly affect their economies. (Dworsky 2002)

\subsubsection{Human Capacity}

The significant lack of skilled professionals in local water and development sectors has been noted by several researchers. Government representatives see this lack as a critical need to support locally determined governance arrangements. Falkland (2002) observes that the capacities of national water agencies require strengthening in many areas, including water resources assessment and monitoring capabilities, water planning, and appropriate WASH technology. Government representatives have articulated significant deficiencies in their national and local capacity for tasks relating to water management, and that these deficiencies prevent them from conducting proper planning, development, and sustainable management of their limited and vulnerable water resources.

White (et al. 2006) observes that small island nations often have very limited numbers of trained water resource and supply specialists, and the day-to-day tasks they have to face are significant and erode their ability for strategic planning and action. White (et al. 2007a) also notes that urban and rural water supplies are frequently under different organizations, but urban water suppliers have the concentration of water expertise and resources.

Dworsky's (2002) summary of opinions from representatives of governments across the Pacific identifies that insufficient institutional capacity is a significant constraint to sustainable water and wastewater management by utilities. A number of constraints relate to human-capacities: limited technical expertise, weak institutional bodies, demoralized and unmotivated staff, insufficient training opportunities, poor staff retention, and weak leadership of utilities (Dworsky 2002). The shortage of managerial and technical 
skills was a major development constraint. The human resources and institutional capacity in the water and wastewater utilities, specifically, and in the Pacific Island countries generally, remained extremely weak, caused by the small labor market and limited local education opportunities. Weak human and institutional capacities were identified as the most significant stumbling block to achieving not only sustainable water and wastewater utilities, but efficient social and economic systems. Weaknesses range from the weak capacities of parliaments to debate policy issues and to effectively oversee government administration, to the limited professionalism of the civil service, the weak capacity of sectoral agencies to regulate and manage critical public services, and the ability of the private sector to engage the necessary business and technical skills (Dworsky 2002).

Dworsky (2002) adds that while the shortage of skills in the smallest and remotest Pacific Island countries is to be expected, the significant effort that has been expended in building skills in the larger Pacific Island countries, such as the Fiji Islands and the Melanesian countries, has not led to sufficient durable capacity. An important lesson from capacity building efforts in the Pacific so far is that it is a long-term task requiring sustained attention and investments; given the weak base in most countries, shorter-term initiatives are overly ambitious (Dworsky 2002).

Poustie and Deletic (2014) identifies human capacity as an important barrier to achieving sustainable urban water management in Vanuatu, recommending skills development regarding both technical and socio-institutional capacity. Limited knowledge among national and local government staff, specifically a lack of trained engineers, has resulted in heavy dependence on external sources of knowledge when technical decisions are required (Poustie and Deletic 2014). This lack of local knowledge of possible solutions-coupled with zero experience in design, construction, and implementation of structural measures-explains why, despite studies and recommendations, nothing has been achieved in improving Port Vila, Vanuatu's urban water infrastructure over the past 14 years (Poustie and Deletic 2014). This research notes that local stakeholders have recognized the need and expressed a desire to develop their local capacity for sustainable water management, including a desire to learn from countries further developed than theirs, and to adapt Vanuatu's water management pathway so as to "leapfrog" mistakes (Poustie and Deletic 2014; see also chapter 7). Although international consultants acknowledge their high level of knowledge, the nature of consultancy projects makes it impossible to adequately train or develop the capacity of national staff (Poustie and Deletic 2014).

Related to the use of international consultants to build local capacity, Aitaro et al. (2007) note that technical reports prepared by consultants-as part of their 14-country program-are rarely used or even read by local stakeholders. Reasons include (i) the reports weren't communicated appropriately for this audience, and (ii) they do not acknowledge existing capacity and contribution to technical content. Capacity building programs are often executed by long-term consultants who are technical rather than capacity building specialists, and who end up doing the work themselves rather than transferring skills (Dworksy 2002).

Keen (2003) observes that many national and regional workshops have tried to build capacity on particular aspects of water management, but the large number of narrowly focused and disparate workshops have led to the risk of Pacific Island countries spreading their efforts too thinly across many different water issues, and making it even more difficult to have an IWRM system. Dividing water management into manageable areas of concern related to the expertise of host organizations has resulted in a strong emphasis on technical issues and capacity building, with a relative neglect of policy issues and the role of 
the public. However, Keen notes that donors have a significant influence over how water management capacity is developed, and that some donors take a more holistic perspective rather than technical focus. White et al. (2006) and Aqorau (2016) also note the important influence of donor agendas on water management.

White et al. (2006) identify that the short-term, relatively narrow focus of aid projects is at odds with the nature of support to Pacific Island countries required to achieve integrated, coordinated, and skilled water sectors. Long-term (e.g., 20-year) phased approaches are needed to tackle priority problems systematically.

As observed by Dworksy (2002), many challenges relating to limited integration, poor coordination, and weak human capacity were identified decades ago (e.g., Falkland [1992]). Although an assessment of the current situation hasn't been undertaken since 2002, the issues identified in 1992 are likely relevant 30 years later.

\subsection{Ways Forward}

\subsubsection{National Sector Reform and Leadership through National Water Apex Bodies and Community Engagement}

A number of publications outline the importance of and recommendations for nationally driven governance reforms (e.g., Hill [2002]; SOPAC [2008b]). However, White et al. (2007a) conclude that water policies and reforms derived from upper-middle-income countries (UMICs) tend to conflict with traditional rights and values of small island countries, and so will be adopted only when the need for them is recognized and island communities adopt and own them.

Pacific Island countries have progressed differently in establishing key governance structures and frameworks that support strategic planning and IRWM, such as forming a water apex body or revising policy, legislation, and action plans (SOPAC 2008b). Most have established a national water apex body (NWAB) to improve coordination of water resource management (SOPAC 2008b). The NWAB model provides a forum for planning of national policy, legislation, strategy, and action to improve water resource management as an important part of overarching national development planning. A NWAB can take many forms, building on existing structures or combining with other committees. The essential functions of a NWAB (SOPAC 2008b) include the following:

- Prepare an overarching national vision and action agenda for sustainable water resource management that formulates sound policies and laws, builds institutional capacity, and manages all the information generated in the planning, management, and reform process.

- Guide and coordinate water resource planning, management, and reform processes within overarching work plans and action frameworks for action so results are consistent with agreed expectations. $^{3}$

${ }^{3}$ Developing national instruments includes national visions, policies, plans, and legislation appropriate to each island country, while considering each citizen's social, economic, environmental, and cultural needs. 
1. Facilitate dialogue among government, nongovernmental, and external actors (such as civil society, communities, and development partners) for input, support, and contributions to joint, strategic, and coordinated action to improve water resource management.

- During a 2008 workshop of government representatives (SOPAC 2008b), participants recognized that stronger NWABs have greatly contributed to highlighting water as a political priority; improving transparency and coordination within the sector; formulating government objectives and policies for the sector; improving access to resources for improving water resource management; and addressing related issues such as climate change adaptation. However, these government representatives highlighted the following areas as needing improvement: stakeholder involvement, public awareness, and communications; and a clear mandate and high-level endorsement and support to keep driving the process forward.

SOPAC has developed guidelines to develop and strengthen policy processes, structures to enhance the intersectoral involvement, and coordination of regional, national, and local stakeholder groups in water resource management. National building blocks for Pacific Island countries have been identified by regional stakeholders as being useful in underpinning sustainable water governance (Hill 2002): water sector assessment; agreement on a broadly based water vision; development of water action agenda and plans; design of capable institutions; integrated investment plans; regional support; active initiation of dialogue with investors and donors; and awareness raising and genuine consultations with island communities.

These building blocks provide a useful general framework for improving national water governance, including integration, coordination, and capacity. However, national leadership must be underpinned by genuinely participatory processes to build the necessary support and ownership of visions and action plans. As such, a high-quality participatory framework should be adopted at the national level to allow for open participation of communities in sustainable water and wastewater management (Carpenter and Lawedrau 2002).

In Pacific Island countries, with many remote rural communities and weak state and private sector capacity to manage rural systems, communities will continue to play critical roles in managing their own water systems, and contributing to larger-scale water resources protection (see also chapter 8). Customary rights include water ownership in many cultures, further requiring good participatory governance so that water resources might be shared.

Engagement with urban communities is essential, but much less effort has occurred in engaging urban communities in water management and conservation (Keen 2003). Forms of community engagement, such as community-based urban water monitoring and catchment management within urban and periurban settlements-popular in places such as Australia-have not been implemented widely in the Pacific (Keen 2003). Barrington et al. (2017) describe a model of participatory governance focused on urban and peri-urban informal settlements in Melanesia in which an informal forum of informal settlers, government, utility, and other enabling actors regularly meet to identify collective actions to improve WASH situations for informal settlements. In this model, the role of external agencies and organizations is not limited to their formal roles and responsibilities, but rather, emphasizes the broader technical and nontechnical support and advice that these actors can provide to participatory development (Barrington et al. 2017). 
Private sector engagement in governance is likely to be increasingly necessary, because businesses play increasing roles in water and sanitation service delivery (domestic, commercial, agricultural), and because major water users recognize their role in local water security. Models such as water stewardship provide a framework for private sector contribution to water security; variations of that model to catchment-scale partnerships on local water security may allow catchment stakeholders to identify catchment and local actions to improve water security.

In some American-affiliated Pacific islands, public-private initiatives for catchment and watershed management were initiated in early 1990s. They formalized joint initiatives between government agencies and private landowners including producers, environmental organizations, and local water supply boards; the PPS in Hawaii occurred with the National Park Service (Evensen 2010). The partnerships vary in size, number of partners, specific goals and funding, but all agree on the value and need to protect forested areas as sources of water, native species, and valuable ecosystems. The partnerships seek to implement best management practices for catchment management (Evensen 2010). The applicability of such PPSs working on locally relevant issues to other Pacific Island countries is not clear but could be explored.

\section{Decentralizing WASH Governance in Papua New Guinea}

A recent review of governance reforms relating to WASH service delivery in Papua New Guinea determines that the recently created District Development Authorities (DDAs) present a strong opportunity to decentralize and localize the government's new national WASH policy (World Bank 2017). The DDAs are an administrative service delivery mechanism created to work with provincial and local governments, supporting local government and provincial agendas within national systems and priorities, thus effectively decentralizing service delivery. The review articulates a complex arrangement of government instruments linking planning and budgeting between local, provincial, and national government ministries, and outward to the Sustainable Development Goals (SDGs), noting that the complexity is appropriate and logical. The review reports that these reforms are leading to many improvements in coordination, accountability, clarifying roles and responsibilities, stronger subnational governance and service delivery, and supporting the engagement of local people in determining and addressing priorities. The larger size and sociocultural complexity of Papua New Guinea in comparison with other Pacific Small Island Developing States (SIDS) mean these reforms may not be directly applicable to other places, but there may be lessons in mechanisms for decentralizing governance for Pacific SIDS due to their dispersed populations.

\section{Micronesian Approach to IWRM}

In 2011, the Marshall Islands, the Federated States of Micronesia, and Palau agreed to develop a collaborative, subregional solution to address fragmented water supply and sanitation (WSS) efforts. National water summits would be held to discuss WSS needs, benchmark the state of WSS management, and identify policy gaps. The summits would be a first step toward developing national water policies in each country. (WHO 2016)

The nations agreed to each hold national water summits on World Water Day to discuss priority issues and possible solutions and policies. Summit participants have included traditional leaders, community representatives, civil society, and government representatives. The national water summits have engaged leaders at the highest levels and secured commitments to the development of solid policy platforms and investment plans for WSS. 
The successful subregional approach is continuing beyond the national summits, with a Micronesian Water Committee established as a permanent working group of the Micronesian Chief Executives Summit (MCES). With the support of the Pacific IWRM program, MCES has established the Federated States of Micronesia's Water for Life Campaign, including the Pacific Blue Water Ribbon and Blue Ribbon Day to be celebrated each World Water Day. The subregional approach and country-specific programs for the North Pacific have been successful in mobilizing IWRM solutions through a process that sustains country motivation and enthusiasm in an atmosphere of Micronesian camaraderie and achievement at all levels.

\subsubsection{Regional Support and Synergies}

While acknowledging the critical need for country-specific governance arrangements and instruments that consider local cultural, social, environmental, and economic situations, most of the analyzes reviewed recommend regional actions as being an effective means to improve sectoral capacity, coordination, and integration (Carpenter and Lawedrau 2002; SOPAC 2008b; White et al. 2007b).

Keen (2003) notes that Pacific regional agencies have been overall very effective in supporting their local counterparts through technical assistance, training, and advisory services. Their established track record could be used to promote better institutional integration and stronger policy formulation by fostering linkages across levels of governance and between government agencies such as water, urban planning, and health.

White et al. (2007a) identifies that regional water organizations, through fostering self-support, are key to developing island-adopted and -owned solutions. Water management problems are common to many Pacific Island countries, and skilled human capacities are limited in small island countries. Therefore, regional pooling of expertise through partnerships with adequately resourced, long-lived regional organizations has the potential to overcome capacity. Regional water organizations such as the South Pacific Community, the Pacific Water Association, and the Secretariat of the Pacific Regional Environment Program can play key roles in building self-reliance in small island countries if supported to maintain longterm relationships, but aid donors have overlooked or ignored the potential of such regional organizations (White et al. 2007a).

\section{Box 4.1: Donor Cooperation in the Pacific}

The Pacific Region Infrastructure Facility (PRIF). Established in 2009, PRIF comprises major donors engaged in the region: the Asian Development Bank (ADB), the World Bank, the European Union (EU), the European Investment Bank (EIB), the Ministry of Foreign Affairs and Trade (MFAT), the Department of Foreign Affairs and Trade (DFAT), and the Japan International Cooperation Agency (JICA). PRIF seeks to deliver (i) coordinated support by bringing together support from multiple development agencies to make assistance go further and make a bigger difference; (ii) improved prioritization based on the national strategies of Pacific Island countries and sector planning policies; (iii) better policy frameworks by ensuring that infrastructure investments are efficient, effective, and affordable; (iv) sustainable and resilient infrastructure by ensuring effective maintenance of infrastructure and appropriate design responses to natural disasters and climate change impacts; and (v) improved capacity of Pacific Island countries to plan, develop, manage, and maintain their infrastructure. PRIF provides an institutional and procedural framework to enable its members to improve development effectiveness and donor coordination in the Pacific aligned with the Cairns Compact on Strengthening 
Development Coordination in the Pacific 2009 (Forum Compact), the Paris Declaration on Aid Effectiveness 2005 and the Accra Agenda for Action 2008. (PRIF website ${ }^{4}$ )

Secretariat of the Pacific Community (SPC). SPC is the principal scientific and technical agency supporting development in the Pacific; it is owned and governed by 26 members including all 22 Pacific Island countries and territories. Its work focuses on 10 core areas, including two of particular relevance to water: (i) climate change and environmental sustainability and (ii) geoscience. There are three climate change adaptation programs with IWRM initiatives under climate change and environmental sustainability, funded by the EU and the French and the German government, respectively. Technical support for water resources, WASH services, and water governance is under geoscience. It includes support in monitoring the hydrological cycle, monitoring of water quality, designing and implementing water safety plans, and supporting the Pacific Partnership Initiative on Sustainable Water Management (a voluntary partnership of water and wastewater stakeholders in the Pacific region).

Evensen (2010) reviews water quality protection and management in the American-affiliated Pacific islands, with emphasis on the activities of a regional water program supported by the U.S. Department of Agriculture. Despite diverse cultures and conditions across the islands, there is much in common among ground and water issues and many benefits derived from building relationships, sharing resources, and working for mutual benefits. Shared knowledge and resources include approaches and resources to build community understanding of safe rainwater harvesting, and safe animal and human waste management.

SOPAC (2008b) acknowledges the need for regional support for NWABs and national activities. Regional frameworks, such as the Pacific Regional Action Plan on Sustainable Water Management, the Pacific Wastewater Framework for Action, and the Pacific Framework for Action on Wastewater, Water Quality and Health, can provide guidance for national policies and programs. See box 4.1 for examples of donor cooperation in the Pacific. The Pacific Partnership Initiative on Sustainable Water Management was a voluntary partnership of water and wastewater stakeholders with a common goal of achieving sustainable water and wastewater management in Pacific Island countries.

Since 2003, the Pacific Regional Action Plan on Sustainable Water Management has been the key strategic document underpinning regional investments in water. It includes plans to undertake a systematic, phased approach to filling knowledge gaps; develop national WSS policy, legislation, and long term-action plans; establish a whole of government and community national coordination committee; form village water and groundwater protection committees; foster behavioral change and information exchange; and identify long-term priorities and agendas for the WSS sector (White et al. 2006). The regional plan notes the similarities of human resource needs for water management across Pacific Island countries that could be addressed at regional and national levels through targeted training and capacity building (SOPAC and ADB 2003). For example, collaboration among water utilities with a regional partnership could reduce unaccounted-for water (UFW), significantly improve the sustainability of utilities, and reduce the need for developing new water resources (SOPAC and ADB 2003).

Documents note that regional agency support is a recommended mechanism to improve local capacity, for both technical and nontechnical needs, including integration and coordination. While there is strong evidence to support to usefulness of that, Aitaro et al. (2007) observes that better collaboration between

${ }^{4}$ https://www.theprif.org/what-we-do 
regional organizations (including members of the Council of Regional Organizations in the Pacific [CROP]) would strengthen regional support mechanisms; some CROP agencies appear to compete rather than collaborate for funds, and some appear to duplicate areas of work.

\section{Box 4.2 Twinning Arrangement with Water Papua New Guinea and Hunter Water Utility}

In the early 2010s, Water PNG, the urban water utility responsible in Papua New Guinea for the provision of WSS services in 20 provincial towns, was experiencing difficulties in fulfilling its mandate because of insufficient water availability, difficult hydraulic conditions, customer reluctance to pay, and weak management information systems. Starting 2011 and for a two-year period, Water PNG entered a twinning arrangement with the Australian water utility Hunter to help address these major challenges. Under this arrangement, facilitated by the ADB, consistent water quality testing was achieved, nonrevenue water (NRW) was reduced by 10 percent, defective assets were replaced or refurbished, all assets were recorded in Water PNG's asset register, and a master plan for water sources, water distribution, wastewater collection and treatment was prepared.

Sources: ADB 2014

The importance of having long-term regional (and other external) support mechanisms is stated in several analyses. Most Pacific Island countries have a low starting capacity for water management and development; in addition, the need for participatory governance requires time for relationships to foster and collective action to emerge. There is also a need for local capacity processes to include local stakeholders given their necessary engagement with participatory governance.

\subsubsection{Building Local and Regional Capacity}

Carpenter and Lawedrau (2002) identify similarities in the capacity needs of Pacific Island countries that can be addressed regionally through targeted training and capacity building. However, some islandspecific regional training programs may need to be developed for some aspects of water management and to concurrently build capacity of local communities, which have a significant role in using and managing rural water resources. To build capacity, sector stakeholders articulate a need to (i) enhance education and career development opportunities in the water sector (such as scholarships for advanced training courses, including distance learning); (ii) regional or in-country training workshops on targeted need areas; (iii) training courses in partnership with tertiary institutions; (iv) twinning or interchange of professional and technical staff between different islands; and (v) active involvement in appropriate research and implementation projects. (Carpenter and Lawedrau 2002).

Provision of targeted technical capacity is likely to remain an important requirement of capacity building for the near to intermediate future. The Keen (2003) provides recommendations on the concurrent development of nontechnical capacity. Policy makers should consider locating technical capacities within a large integrated water management framework.

Although the use of consultants and external advisers is inevitable, given the constrained human resource capacity in the Pacific, these engagements should be more carefully designed (Dworksy 2002). If the consultant's mandate is to build capacity, rather than fulfill a line function, technical expertise is not a sufficient qualification for the job. Rather, proven success in transferring skills and supporting participative capacity building should be essential requirements. (Dworsky 2002). Identifying and sharing water resource management knowledge and skills across all stakeholders at national and regional levels will contribute to national capacity and make more efficient use of limited human capacity, especially regarding specialist technical expertise (Carpenter and Lawedrau 2002). Keen (2003) and White (et al. 
2006) note the important influence donors have on approaches to water management: support for improving capacity, integration, and coordination is preferable from donors with holistic perspectives on water management rather than narrower technically focused donors.

\subsection{Gaps in Analytical Knowledge}

There appears to be sufficient general analytical knowledge to support integration and coordination, including general understandings about the links between water and other systems. However, countryspecific information, developed with local stakeholders, that identifies environmental and administrative links throughout water systems, and between water and other systems (such as health, agriculture, natural resources) would likely enhance the awareness and ability for national agencies to identify and manage integration, and provide a basis for coordination.

The most significant gap in achieving integrated and coordinated water governance appears to be the human capacity among national agencies to drive effective integration and coordination. Some targeted capacity building has taken place in the past decade. However, this rapid review does not identify any recent capacity needs assessments for the water sectors of any Pacific Island countries or the region. Previous capacity building was mostly technical. As such, gaps likely remain in nontechnical areas, such as processes for integrated and coordinated governance.

Some Pacific Island countries have universities and vocational training centers, offering a range of technical and nontechnical skills related to water management and development. However, this rapid review does not identify assessments of the scale, scope, or effectiveness of these mechanisms to develop capacity.

This review has not located specific assessments of sector capacity that might reveal the nature and causes of the deficiencies, which could include insufficient numbers of skilled workers (due to insufficient motivation to enter or stay in the sector, or insufficient training opportunities), or the insufficient skills of existing workers (or both). The GLAAS survey (2014) contains a section identifying the main drivers of insufficiently skilled workforces. This wasn't completed by Pacific SIDS, but is a simple tool that could elicit a stakeholder-based understanding of causes of insufficient human capacity.

Most documents identify the value of regional support for country-led sector reforms and water management, including sharing and building human capacity, to improve local water management and development capacity. However, this rapid review does not identify evaluations of past regional support mechanisms. Identifying strengths, weaknesses, and opportunities for improved regional support would guide future investments in regional support. Finally, there are gaps relating to analysis and identification of effective and feasible participatory governance arrangements, which are likely to be country-specific, or at best specific to cultural groupings (e.g., rural Melanesia, urban Melanesia, and rural Polynesia). 


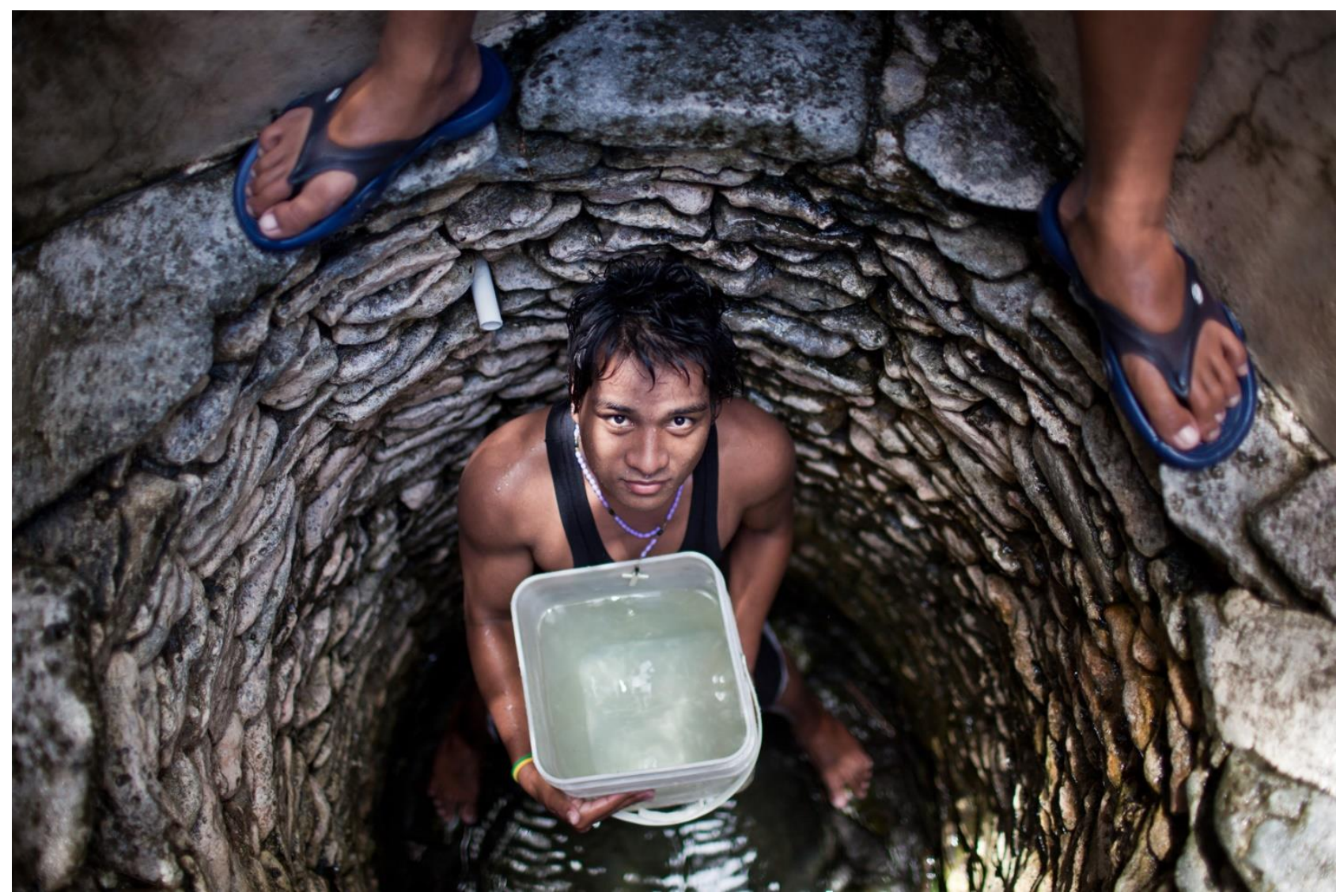

(Credit: World Bank)

\section{WASH Service Delivery}

\subsection{Nature of the Challenge}

\subsubsection{Overview and Assessments of Current Services}

Analysis of the 2015 Joint Monitoring Programme (JMP) data indicates that only half of the population in the Pacific region use improved drinking water sources, whereas one-third use improved sanitation. This means that two-thirds of the region's population rely on unprotected sources of water and unsanitary means of excreta disposal that pose serious risks to health (WHO 2016). The Pacific region made little progress toward achieving the Millennium Development Goals (MDGs) target for WSS during the 25-year period of those targets. Overall access to improved sanitation increased by 2 percent (to 31 percent), and to improved water by 6 percent (to 52 percent) (WHO 2016). Maps 5.1 and 5.2 provide an overview of current access to basic water supply and sanitation across the Pacific region.

The current trend will lead to a total population served in 2030 equal to one-third of the amount required to attain the universal coverage with basic sanitation. Rather than providing services to an additional 56000 people annually as occurred from 1990 to 2015, achieving universal coverage from 2015 to 2030 will require that this number is increased twelve-fold to 650,000 additional people served annually (WHO 2016). These figures, although distorted to some extent by the weight of Papua New Guinea in regional statistics, suggest that the gap to universal service delivery is large, and the pace of improvement has been very slow. They have been driven, to a large extent, at a regional level, by fast population growth in Melanesian countries. 
For urban areas, utility benchmarking information is available for all countries and territories of the Pacific through the International Benchmarking Network (IBNET) program, hosted by the Pacific Water and Wastewater Association. Although several Pacific small island developing states (SIDS) have achieved high levels of coverage with improved drinking water sources, there is little information about the quality and safety of the water delivered to the population (WHO 2016). These analyses confirm the gap to universal service delivery is large, and the pace of improvement has been very slow. Improving service delivery to provide health and well-being outcomes arising from universal, sustainable, and safe access to water supply and sanitation (WSS) is a significant challenge.

According to the WHO (2016) analysis, achieving the Sustainable Development Goal (SDG) targets in Pacific Island countries will require increased government and development partner resources, as well as new sector policies and service delivery strategies. The issue of sector policies is addressed in chapter 4 . The infrastructure cost of achieving universal coverage of basic drinking water supply by 2030 is about US\$83 million per year, approximately 0.35 percent of the region's gross domestic product (GDP), and for universal adequate sanitation, about US\$80 million per year, representing 0.33 percent of the region's GDP (WHO 2016). Limited data are available regarding the volume and strategies for investments in WSS service delivery.

Map 5.1 Access to Basic Water Services in the Pacific Island Countries

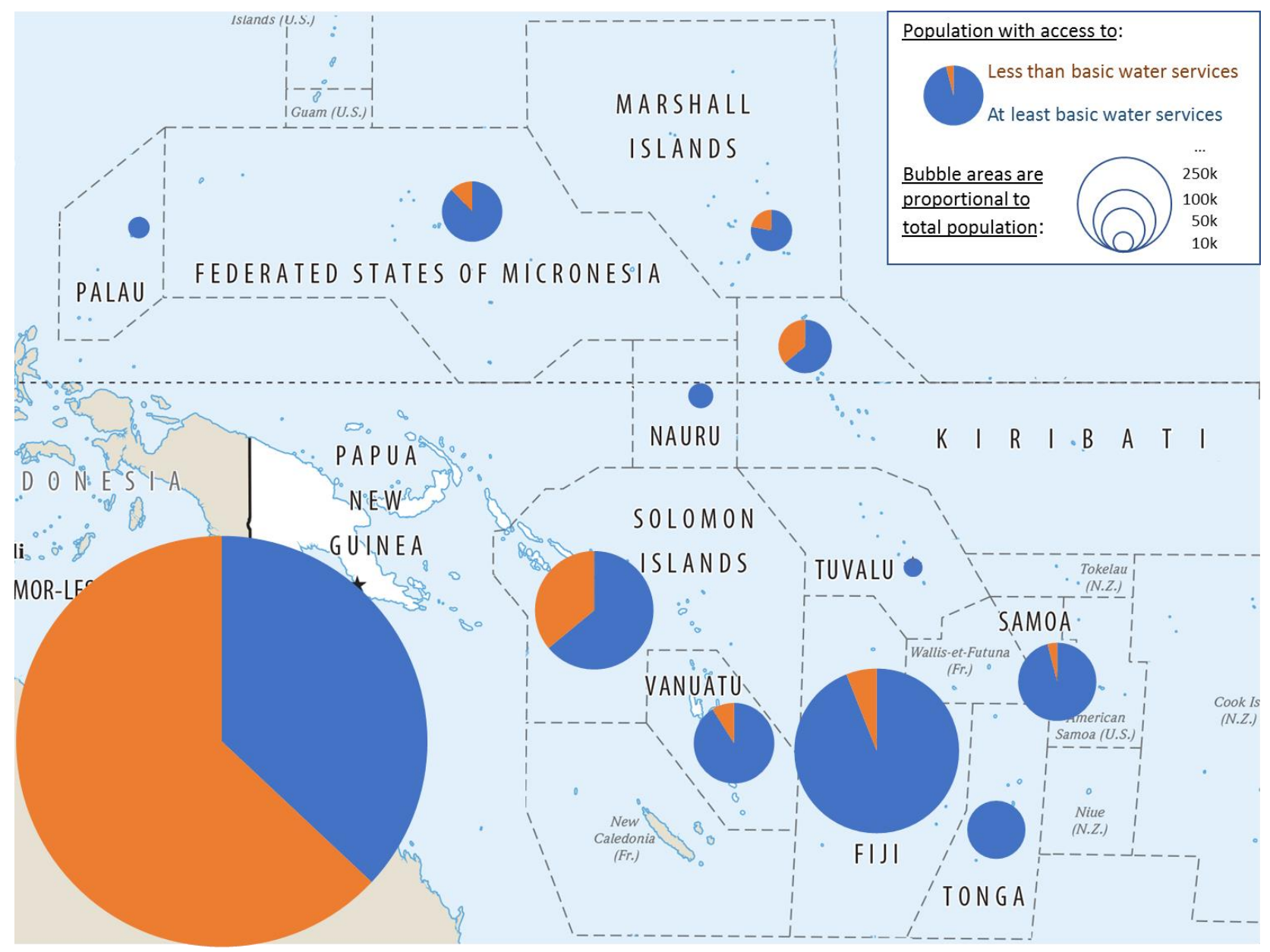

Sources: WHO/UNICEF 2017.

Note: "Access to basic water services" refers to Sustainable Development Goal (SDG) 6.1. 


\section{Box 5.1 Non-Pacific SIDS-Levels of Access to WSS in the Pacific (outside of PNG) consistent with SIDS Globally}

At least 82 percent of the global population of SIDS globally have access to basic water (63 percent, rural, compared to 94 percent, urban) services, a level. In comparison, 50 percent of Pacific SIDS population have access to basic water, 86 percent if Papua New Guinea is not included in the calculation. Sixty-six percent of the population of SIDS have access to piped water (42 percent in rural areas compared to 78 percent in urban areas, down from 85 percent in the latter in 2000). Sixty-eight percent of the population have access to basic sanitation services (48 percent, rural, compared to 80 percent, urban). In comparison, 32 percent of Pacific SIDS population have access to basic water, 70 percent without Papua New Guinea. Thirty percent of the population of SIDS have access to sewers, and 50 percent of wastewater collected undergoes treatment (but this represents wastewater for only 14 percent of the overall population). However, the gap in access to WASH services between rich and poor for several SIDS is the highest globally (Haiti, Belize, Suriname, Barbados, Jamaica, Comoros, St Lucia, and Timor-Leste). Most countries in the Caribbean report over 95 percent access to water supply. Current problems include quality of service, maintenance and operation of existing infrastructure, aging infrastructure, high levels of unaccounted-for water (UFW), and quality of potable water.

Sources: WHO/UNICEF 2017.

Map 5.2: Access to basic water services

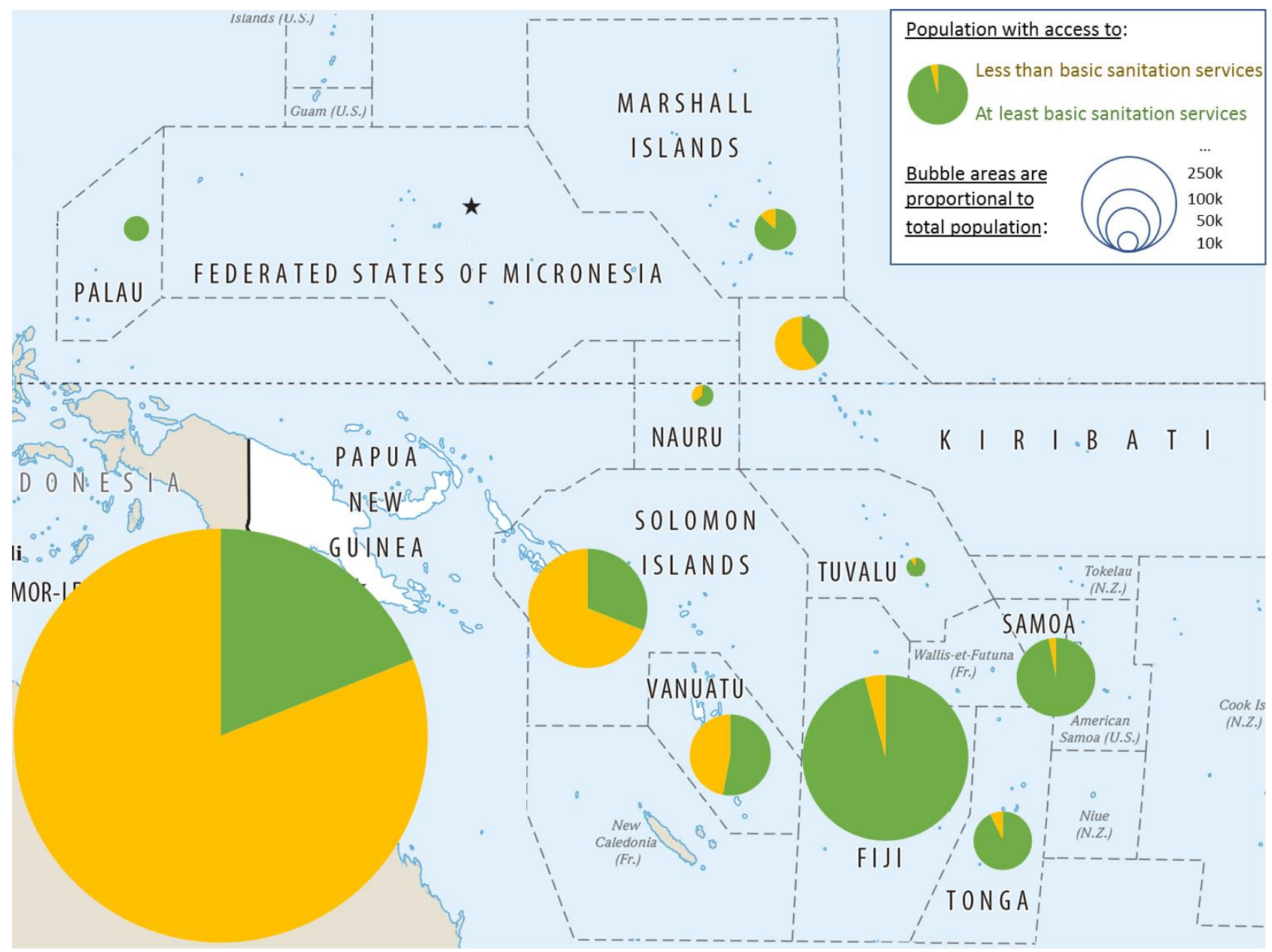

Sources: WHO/UNICEF 2017.

Note: "Access to basic water services" refers to Sustainable Development Goal (SDG) 6.1.

A service delivery assessment of WSS services in Papua New Guinea provides the only population wide perspective on constraints to service delivery discovered in this rapid review (World Bank 2016). The bottlenecks impeding progress relate to the enabling environment (the overall framework for service 
delivery) and implementation. Enabling environment constraints include a lack of policies that clearly articulate a vision, targets, and approaches for WSS; unclear institutional roles in the sector; and lack of budget mechanisms for allocating and tracking expenditures to rural and urban WSS. Implementation bottlenecks include insufficient finance allocated to the sector, weak monitoring systems, and a scarcity of qualified technicians and managers. Contextual factors such as political volatility, poor access due to a lack of roads, no electricity, customary land ownership, and ethnic conflict also hamper progress. Environmental variability, including climate change and disasters, are not identified as key constraints. The assessment provides a basis and motivation for Papua New Guinea's new national WASH policy, which, if implemented well, will address many of the service delivery constraints identified in the assessment.

The service delivery assessment's country-specific analysis can guide where future action and investment are likely to lead to improved service delivery. The recommendations may generally be applicable to other Pacific Island countries; however, because Papua New Guinea is different from its neighbors due especially to its larger population, the applicability to other Pacific Island countries may be limited.

There is a need for a specific approach for water services and management for fragile states. A number of SIDS globally fall into that category, including the Comoros, Haiti, Kiribati, the Marshall Islands, the Federated States of Micronesia, Papua New Guinea, the Solomon Islands, and Tuvalu. In atoll environments, limited land areas severely restrict the ability of service providers or communities to capture and store sufficient freshwater to provide adequate services. Competition with other users-including agriculture and irrigation-environmental variability, and change to rainfall further decrease the security of water sources (White et al. 2004).

\subsubsection{Rural WSS systems}

The disparity between urban and rural WSS coverage is significant: 71 percent of the urban population have improved sanitation compared with 21 percent of the rural population (WHO 2016); 67 percent of the urban population access improved water (piped on premises), while only 9 percent rural population have access to improved water to their household. Rural areas have been neglected in terms of sanitation investments. While the absolute numbers of people unserved has had a relatively small increase in urban areas from 1990 to 2015 , the rural unserved increased by nearly 70 percent (WHO 2016). Defecation on the beach is practiced in many low-density island communities; in higher density populations pit latrines are used, though their location can introduce fecal contamination directly into groundwater (White et al. 2004).

The infrastructure utilized in rural water systems of Pacific Island countries is typically relatively simple technology: extract shallow and deep groundwater (bore pumps, shallow wells, springs); rainwater harvesting; surface water. Some communities, such as on small atolls, utilize more complex technologies such as desalination or reverse osmosis. Basic distribution systems exist, sometimes involving piping to households, but more typically there are communal access points. Due to environmental variability, most rural householders and communities manage a number of water sources concurrently for different uses, seasons, and water availability (Hadwen et al. 2016).

Community-managed water systems remain the dominant system for water supply in rural communities throughout Pacific Island countries. This rapid review has not identified any significant dialogue among Pacific stakeholders regarding an evolution of community-managed water systems to service delivery models, apart from some discussion for Papua New Guinea as part of its service delivery assessment.

Clarke, Feeny, and Donnelly (2014) have evaluated the sustainability of 27 community water and WASH projects completed in the preceding three to five years and concluded that the most important factors affecting sustainability of community systems are the following:

- Alignment of project objectives with needs recognized by community 
- Community ownership of the planning and implementation of aid projects (despite general awareness of the importance of community engagement by project proponents, inadequate community engagement in planning and implementation persists: no community members had input into the design of water or WASH systems in any of the 27 projects)

- Local and appropriate leadership of activity

- Appropriateness of the response to the identified need

- Recognition of the value of gender analysis in all aspects of the response and implementation

- Understanding that differing worldviews affect involvement and commitment to activities

Chapter 6 provides further information about culture and community-managed systems.

\subsubsection{Urban WSS Service Delivery}

Many in urban populations obtain water services from utilities, although it's likely that many households, particularly in informal settlements, rely on other formal or informal means to gain water. Types of sanitation services and facilities used by urban populations vary significantly among Pacific Island countries. Overall, 58 percent of urban dwellers use piped wastewater (PWWA 2016), and 71 percent of urban dwellers use

Photo 5.1: Private restroom is South Tarawa

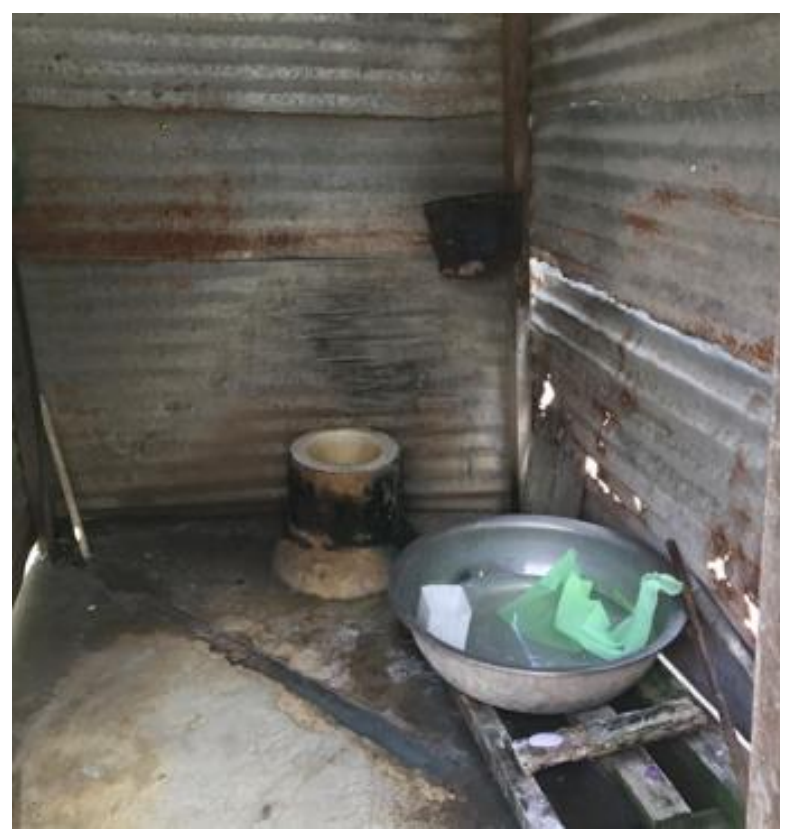

Source: Edkarl Galing/World Bank improved sanitation. The rest uses a range of on-site options, such as septic tanks or various versions of pit latrines (such as in Photo 5.1), and around 3 percent openly defecate (WHO 2016). In small atolls, flushing toilets consume 30 percent to 40 percent of water supplies, and there is little scope for freshwater sewerage systems (White et al. 2004). Majuro and South Tarawa have saltwater sewerage systems, though leakage from these can pose difficulties in dry periods (White et al. 2004). Elsewhere, composting toilets have been trialed, but there are sometimes cultural barriers to their adoption (Crennan and Berry 2002).

Utilities are the major service provider to urban populations, and most countries are required to provide at least water services-and often wastewater or other sanitation services-to all urban dwellers. There are 29 WSS utilities across Melanesian, Polynesian, and Micronesian countries (PWWA 2016).

\section{Service Performance of Utilities}

The Pacific Water and Wastes Association (PWWA) benchmarking report (2016) provides useful insight to the services provided by utilities. All countries have some form of piped water supply for a proportion of the population, but it is available for less than three hours a day in some countries. Across the region, the access to piped water services for urban populations varies from 15 percent to 100 percent (PWWA 2016); and 67 percent of urban population have piped water into the household (WHO 2016).

In general, utilities in countries with lower gross national income (GNI) per capita perform worse than those in the richer countries (PWWA 2016). The lowest coverage is reported from utilities with the lowest water availability. The Water Authority of Fiji (WAF) is actively expanding into suburban and rural areas, so its coverage is higher than 100 percent. The majority of PWWA utilities provide water 24 hours a day, seven 
days a week. The overall trend is positive and has increased from 17 to 21 hours on average between 2011 and 2015. Nauru distributes water in trucks. Marshall Islands companies have to ration water due to significant issues with water availability. Companies in Kiribati and the Solomon Islands are working on expanding their working hours.

UFW is an issue for the majority of the PWWA companies: 16 utilities report UFW, and 12 of these declared a need for significant investments to replace or rehabilitate networks built during 1960s to 1980s. Fresh water availability is not an issue for these companies, and the governance structure of these utilities allows passing the cost of these losses to customers (PWWA 2016). UFW management is considered a complex and expensive task for many PWWA companies, and may require a large financial and technical support (PWWA 2016).

Indicators on per capita production and consumption vary between utilities (PWWA 2016). In the utilities with very limited water resources, water is strongly rationed. In Kwajalein Atoll Joint Utilities Resources (KAJUR), Marshall Islands; the Northern Yap Gagil Tomil Authority (NYGTA) and Southern Yap Water Authority (SYWA), the Federated States of Micronesia; Tuvalu; and Nauru, water consumption is 5-10 liters per capita per day. Losses are very low because every drop of water is costly, and therefore accounted for (PWWA 2016). In larger companies, especially ones operating for a long time, water production and consumption are within the reasonable range of 100-200 liters per capita per day), while in the U.S. and French territories, water production and consumption are high (PWWA 2016). This higher use is because a high proportion of the water is delivered to industrial users. For example, the American Samoa Power Authority (ASPA), American Samoa, sells more than 40 percent of its water to industrial users; Guam Waterworks Association (GWA), Guam, sells 30 percent of water to industries, and Saipan, Northern Marianne Islands, sells about 35 percent (PWWA 2016). Both Papua New Guinea companies sell about 80 percent of their water to nondomestic users (PWWA, 2016).

Across the region, access to piped wastewater services varies from 0 percent to 100 percent. Discouragingly, piped wastewater coverage has reduced across the region in the period 2011-15 (PWWA 2016). This is mostly due to ongoing urbanization in countries such as Fiji and Papua New Guinea (PWWA 2016). Many utilities do not appear to have wastewater treatment facilities. Apia wastewater

Photo 5.2: Butch wastewater treatment plant, Apia, Samoa

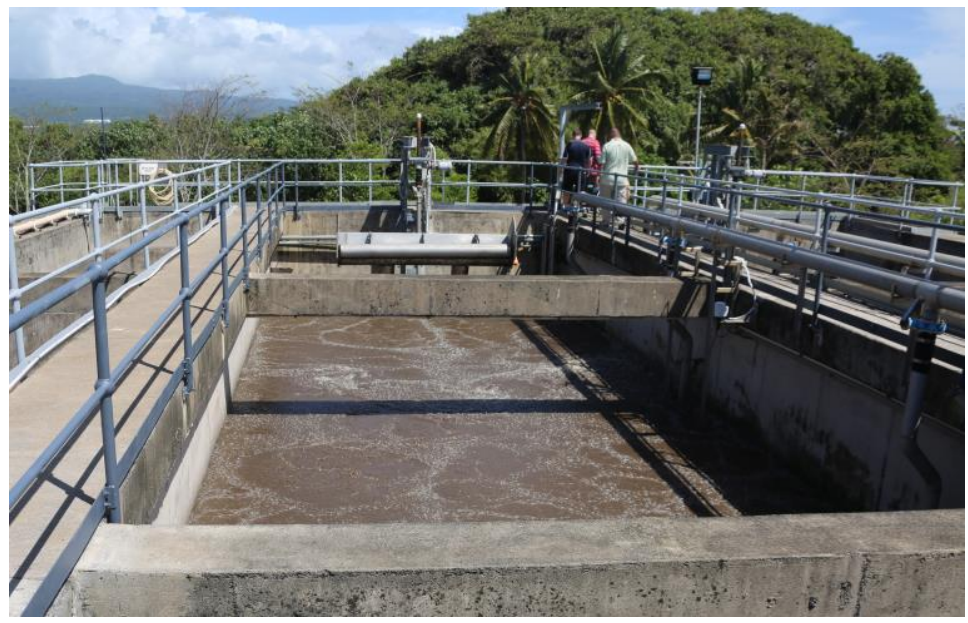

Source: Alexander Danilenko/World Bank treatment plant is shown in Photo 5.2.

\section{Constraints to Service Delivery by Utilities}

Dworsky (2002) provides a detailed analysis of stakeholder consultations and information relating to service delivery by urban water and wastewater utilities:

Insufficient institutional capacity. Lack of data and information systems; insufficient or inoperative equipment; poor equipment maintenance and limited technical expertise; weak institutional bodies; often demoralized and unmotivated staff; poor staff retention; insufficient training opportunities; lack of finances; high cost of service; poor water quality; insufficient water conservation; and poor public relations. In addition, there is often no manager responsible for all aspects of the utility, or the managers lack vision and are unable to articulate a clear vision to the staff. 
Lack of government support. Lack of political will, but often too much unwanted political interference; legislation inappropriate or absent; lack of regulation and no capacity for enforcement; often no coherent national policies on IWRM; and unsolved land issues. There is often fragmented multiple government agency involvement resulting in poor regulatory or policy links between the various sectors; conflict between public service and sustainable utility; political instability; and lack of national long-term planning and commitment. There is no value given to water meters or the establishment of a proper tariff.

Insufficient public support. Inadequate public awareness; insufficient community participation and involvement; and associated lack of appreciation of sociocultural issues.

Additionally, the Pacific Island countries environment (outside of the national capacity) often results in further constraints due to the isolated and fragmented nature of multiple island states including restricted land area; competing land uses; small population base; increasing population density on "capital" islands, and depopulation on outer islands; isolated communities; high transport costs; limited economic development (previous factors unattractive to industry); limited tax revenue generation; poor and costly communication, electricity, and water supply systems (due to low economies of scale); high vulnerability to natural disasters; climate variability, El Niño-Southern Oscillation (ENSO), and sea level rise issues; and lack of coordination among donors, international organizations, and receiving countries. Many of these factors align with those identified for Papua New Guinea in its service delivery assessment (World Bank 2015).

The PWWA Benchmarking report (2016) provides more specific insights to some of the constraints faced by urban utilities in Pacific Island countries. Most utilities are state-owned companies and all have the legal status of a company, except in Niue, and Kosrae, the Federated States of Micronesia, where water is provided by the financially ring-fenced water department. Investment is a significant issue for the PWWA utilities. Some have completed recent investment projects, but others completed their last investment more than 10 years ago. Although this does not necessarily mean that there are no investments at all in the service, as investments might have been financed via Government (PWWA, 2016), it is not clear that sufficient investment to services in occurring. Labor costs are also a considerable issue for utilities. Economies of scale plays a major role in employment: larger utilities tend to have less staff per 1,000 users. (PWWA 2016)

Tariffs are the main source of income for 23 utilities. Water is free for domestic residents in the Cook Islands, Kiribati, and in the Marshall Islands. In some countries, there is one tariff for both the water and sewerage service, and in others there may be a percentage levy on top of the water tariff to cover the service for sewerage (PRIF 2016). For five of these 23 utilities, tariffs are the only source of income; the rest are subsidized by their authorities or other services provided by utilities (e.g., the electricity branch supports water services in PUB Kiribati) (PWWA 2016). The average collection rate for PWWA member utilities is 90 percent, as suggested in Table 5.1; however, it is an issue for seven utilities (PWWA 2016).

Except in part of the U.S. territories, donors and governments cover all costs related to capital expenses and major rehabilitation works. Direct subsidies to cover operations and maintenance costs are provided for all PWWA utilities. In some cases, subsidies cover account receivables due to poor collection. Such subsidies, however, are not guaranteed every year for all PWWA companies: only the Cook Islands, Fiji, the Marshall Islands, Kiribati, and Tonga reported subsidies for every year between 2011 and 2015. There might also be additional indirect subsidies (e.g., waiving of electricity fees). (PWWA 2016) 


\begin{tabular}{|c|c|c|c|c|c|c|c|c|}
\hline Country & Utility / area & $\begin{array}{c}\text { Water } \\
\text { Coverage } \\
(\%)\end{array}$ & $\begin{array}{c}\text { Sewerage } \\
\text { Coverage } \\
(\%)\end{array}$ & $\begin{array}{r}\text { Continuity } \\
\text { of Service }\end{array}$ & \begin{tabular}{|c|} 
Quality of water \\
supplied (samples \\
passing on \\
residual chlorine) \\
$(\%)$ \\
\end{tabular} & $\begin{array}{l}\text { Wastewater } \\
\text { (at least } \\
\text { primary } \\
\text { treatment) } \\
(\%)\end{array}$ & \begin{tabular}{|c|} 
Nonrevenue \\
water $(\%)$
\end{tabular} & $\begin{array}{c}\text { Collection } \\
\text { ratio (\%) }\end{array}$ \\
\hline Fiji & WAF & 86.28 & 35.31 & 20.00 & 92.12 & 24.32 & 45.39 & 100 \\
\hline Kiribati & PUB & 68.54 & 33.17 & 2.00 & 100 & 0.00 & 88.37 & 75.55 \\
\hline \multirow{2}{*}{$\begin{array}{l}\text { Marshall } \\
\text { Islands }\end{array}$} & KAJUR & 90.69 & 100 & 14.00 & 100 & 0.00 & 54.41 & 100 \\
\hline & Majuro & 29.29 & 60.07 & 4.00 & 47.37 & 0.00 & 9.30 & 100 \\
\hline \multirow{6}{*}{$\begin{array}{l}\text { Micronesia, } \\
\text { Fed. Sts. }\end{array}$} & Central Yap & 33.33 & 64.58 & 24.00 & 30.40 & 32.29 & 39.61 & 100 \\
\hline & Chuuk & 22.39 & 20.26 & 24.00 & 100 & 20.26 & 66.75 & 100 \\
\hline & Kosrae & 25.00 & 31.88 & 24.00 & 0.00 & 20.19 & 94.97 & 0.00 \\
\hline & Northern Yap & 100 & N.A & 24.00 & 100 & 0.00 & N.A & 100 \\
\hline & Pohnpei & 61.48 & 100 & 24.00 & 84.11 & 100 & 8.79 & 50.17 \\
\hline & Southern Yap & 100 & N.A & 24.00 & 100 & 0 & 16.67 & 0.00 \\
\hline \multirow{2}{*}{$\begin{array}{l}\text { Papua New } \\
\text { Guinea }\end{array}$} & Eda Ranu & 100 & 75.00 & 24.00 & 95.60 & 0 & 39.54 & 100 \\
\hline & Water PNG & 63.90 & 13.75 & 24.00 & 100.00 & 6.88 & 35.61 & 92.36 \\
\hline \multirow{2}{*}{ Samoa } & IWSA & 100 & N.A & 20.00 & N.A & 0 & N.A & 100 \\
\hline & SWA & 81.67 & 6.50 & 24.00 & 94.10 & 6.50 & 58.98 & 74.42 \\
\hline $\begin{array}{l}\text { Solomon } \\
\text { Islands }\end{array}$ & SIWA & 61.64 & 6.23 & 22.60 & 89.72 & 0 & 57.79 & 100 \\
\hline \begin{tabular}{|l|} 
Tonga \\
\end{tabular} & TWB & 100 & N.A & 24.00 & 97.52 & 0 & 53.70 & 86.88 \\
\hline Tuvalu & $\mathrm{MUI}$ & 94.00 & N.A & 24.00 & N.A & 0 & N.A & N.A \\
\hline Vanuatu & Unelco & 74.95 & N.A & 24.00 & 100 & 0 & 18.20 & 0.00 \\
\hline
\end{tabular}

Sources: IBNET; PWWA. 2013-2017

Note: Figures are provided for the latest year of the 2013-17 interval for which data are available. KAJUR = Kwajalein Atoll Joint Utilities Resources. IWSA = Independent Water Schemes Association. MUI = Ministry of Utilities and Industry. PUB $=$ Public Utility Board. SIWA = Solomon Islands Water Authority. SWA = Samoa Water Authority. TWB $=$ Tonga Water Board. WAF = Water Authority Fiji. N.A. = not available.

\section{Other WSS Services for Urban Populations}

WHO (2016) reports that 23 percent of urban populations do not have piped water to their household. That report does not identify alternative sources used by the remainder of the population, but anecdotally, household rainwater tanks appear to be common in urban centers across the Pacific Island countries. It is not clear whether informal settlers are included in these estimates. Onsite septic tanks are thought to be widely used throughout urban centers. However, this rapid review does not find data describing (i) onsite septic tanks, (ii) service delivery models surrounding septic tanks, or (iii) targeted discussion on fecal sludge management.

\subsubsection{Equity and Inclusion}

Apart from data distinguishing urban and rural populations, data describing access to WSS services by marginalized people are not available for Pacific Island countries. From some targeted studies (some unpublished), many people living within urban areas, or on the boundary of urban and rural jurisdictions, have limited access to safe WSS services, particularly in Melanesian countries (Barrington et al. 2016; Schrecongost and Wong 2015; WSP 2014). Most settlement residents rely on shared and unimproved sanitation facilities, such as hanging toilets that are "straight-piped" to drains, streams, or pit latrines that tend to fill and flood regularly (Schrecongost and Wong 2015). Waste removal, conveyance, and treatment are absent in all settlements with very few exceptions (Schrecongost and Wong 2015). Regarding access to piped water services, access rates vary among Melanesia countries. Some have formal utility connections at the house, others utilize communal tap stands provided by the utility, and many typically access water from open wells, boreholes, collected rainwater, or surface water sources. 
Utilities underprovide these services, partly because they do not have a clear obligation to serve informal settlements and in some cities do not have the authority to do so. In four Melanesian countries reviewed, no utility or government body has an obligation or budget to provide sanitation services to informal settlements. Where authorization to deliver services does exist, utilities tend not to prioritize extending services because they are technically, legally, and commercially more challenging to serve relative to formal urban communities. Utility investments are also influenced by internal, government, and donor technical preferences that tend to favor investments in piped water infrastructure systems to formal areas. (Schrecongost and Wong 2015)

In most cases, it is not financially feasible for utilities to extend mains or distribution lines to settlement communities and households under current institutional and financing arrangements. Many utilities struggle to provide acceptable water supply services to existing customer bases, and are unable to cope with the pace of urban growth in formalized communities. Challenges are greater for extending services to settlements with insecure land tenure, those in peri-urban areas that may be more remote or outside of formal utility service districts, and those on land that is technically challenging to reach with traditional infrastructure. Settlers may vandalize distribution lines for illegal connections (or there is the perception that they may), which increases nonrevenue water (NRW) losses. It is also more difficult to collect connection fees and enforce bill payment from settlement customers by relying on traditional customer engagement models

Photo 5.3: Relying on solar water disinfection in South Tarawa's informal areas

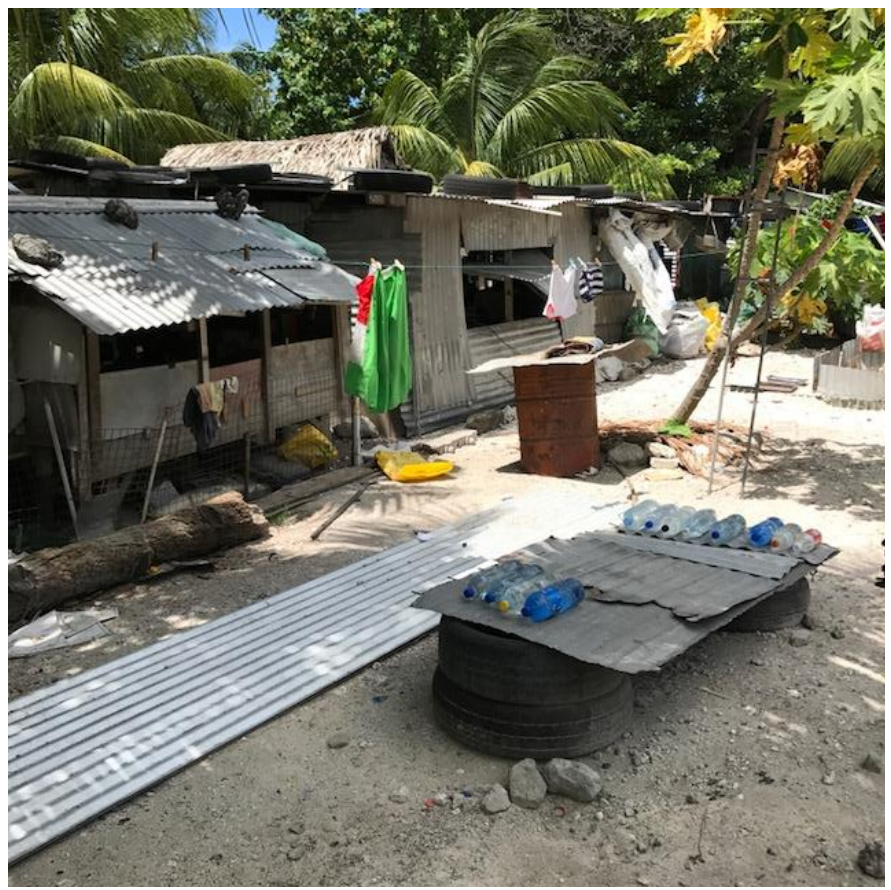

Source: Stephane Dahan/World Bank alone. (Schrecongost and Wong 2015)

In some countries, governments view settlements as temporary and believe access to services encourages permanency and settlement growth (e.g., in the Solomon Islands and Papua New Guinea). The government view is that settlers should return to their rural villages. However, many settlers are second- or thirdgeneration inhabitants and would therefore have difficulty adapting to or being accepted back into village life. (Schrecongost and Wong 2015)

Even if utilities were to provide services to informal settlements, without pro-poor strategies, it is not clear that many of these residents would have both the ability and willingness to pay the upfront connection fees. As a consequence, the way informal settlers access water varies within any one settlement. All four broad marketing exchange archetypes are used within any one settlement: market-based (e.g., monetary exchange), nonmarket-based (e.g., gifted or charity), command-based (e.g., regulated utility services), or culturally determined (e.g., gifts, subsidies, or nonmonetary exchanges that occur with other community members). No single method of exchange will likely reach all members of an informal settlement. Therefore, to achieve universal coverage in an informal settlement, WSS systems need to accommodate locally driven exchanges of services and products. (Barrington et al. 2017)

Delivering settlement services requires the involvement of formal local leaders and negotiations with clan leaders. This need for intense community engagement, plus the violent conditions in some settlements, are 
challenges to participatory community approaches, and complicate efforts by the government, utility, and civil society to improve service delivery. The cultural complexity of many settlements adds to the reluctance of government and service providers. (Schrecongost and Wong 2015)

\subsubsection{Disaster Resilience, Recovery, and Rehabilitation}

Chapter 3 addresses the issues of vulnerability of services and recommendations to improve resilience. Considerable effort is being directed to building the resilience of WASH services and systems in urban and rural settings (UNICEF 2017a). However, maintaining service delivery during and following a disaster will be extremely difficult for Pacific Island countries while the constraints in delivering day-to-day services remain (e.g., insufficient human resources and financial resources and aging infrastructure).

\subsection{Ways Forward}

\subsubsection{Finance}

Recommendations from the Pacific Regional Action Plan on Sustainable Water Management (2003):

- Create a better and sustainable environment for investment by both the public and private sector, by developing and implementing national, sector, and strategic plans that identify the economic, environmental, and social costs of different services and develop pricing policies, which ensure the proper allocation of resources for the water sector.

- Establish financially viable enterprises for WSS that result in improved performance by developing appropriate financial and cost-recovery policies, tariffs, billing and collection systems, and financial and operating systems.

- Reduce costs through improved operational efficiency, using benchmarking, development of water-loss reduction programs, and improved work practices.

- Ensure access for the poor to WSS services by developing pro-poor policies that include tariffs with lifeline blocks and transparent and targeted subsidies.

- Achieve sustainable rural WSS services at the community level through developing strategies that incorporate mechanisms for appropriate financing and capacity building.

\subsubsection{WASH Resilience, Water Safety, and Security}

Hay and Sem (2000) note the following adaptations with relevance to water service delivery models in Pacific SIDS. Improved management and maintenance of existing water supply systems is a high priority response, due to the relatively low costs associated with reducing system losses and improving water quality (compared with developing new water resources). Centralized water treatment to improve water quality is viable for most urban centers, but at the village level, more cost-effective measures need to be developed. User pay systems may have to be more widespread. While increasing water storage capacity through the increased use of water tanks or the construction of small-scale dams is expensive, the added security in the supply of water may well justify such expenditure. The development of desalination facilities is an option for supplementing water supplies during times of drought, but in most instances the high costs prevent this being a widespread adaptation option.

In Kiribati, a novel approach to assessing the likely performance of different urban water delivery strategies was used to overcome the issues associated with previous poor decision making, such as lack of consideration of local circumstances and process requirements, and inadequate involvement of affected stakeholders and cross-sectoral coordination. This approach uses participatory modeling involving experts and local knowledge holders. The project demonstrates an approach that usefully maps the causal explanations for why 
developments do not achieve their set goals; the approach may form the basis of assessments when evaluating urban (or rural) water strategies. (Moglia et al. 2008a)

A key strategy to ensure safe drinking water has been the preparation and implementation of drinking water safety plans for urban water supplies and rural- and community-managed schemes (WHO 2016). The water safety planning approach is currently being evolved and customized to suit the context of the Pacific Island countries (UNICEF 2016a). This includes emphasizing the critical need to involve communities in managing the risks relating to water safety and security, because of customary water ownership (see also chapter 6), and the reliance upon community-managed systems.

\subsubsection{Equity and Inclusion}

Development literature indicates the most effective strategy to overcome exclusion of some people is to strengthen social cohesion (OECD 2012); efforts targeting strengthening of social capital, mobility, and inclusion lead to cohesive societies and communities. Using genuine participatory approaches to water management and service delivery with Pacific SIDS communities would further strengthen social cohesion, and is in line with recommendations to share water management with communities for social and cultural reasons (e.g., customary water ownership) (White et al. 2006).

Tapping Connections between Water \& People (Crennan 2004) is a useful guidebook for field workers when working with rural communities to install and maintain WSS systems. It is grounded in processes that engage communities in the equitable and sustainable protection of public health and natural assets.

Utilities in Pacific SIDS are trialing different ways to engage with impoverished urban communities, including informal settlements. These methods include different pro-poor strategies to support piped water connections, though these are not well-documented. The research on WASH marketing systems in Melanesian urban informal settlements (Barrington et al. 2017) highlighted the need for utilities to consider offering, allowing, or supporting nonmarket-based systems for water services to householders in these communities. A single approach, such as market-based provision of water, will not be socially inclusive and will leave some in the community without access. Some community service organizations (CSOs) have worked or are working with urban informal settlements to improve their sanitation, using such approaches as nonsubsidy, behavior-based strategies (e.g., community-led total sanitation [CLTS]) to sanitation marketing. The latter is being trialed by the Live \& Learn Environment Education organization in the Melanesian countries, with the principle of community-based sanitation enterprises. The program is due for completion 2018.

\subsubsection{Urban WASH Services}

Dworksy (2002) describes the main functions and components that sustainable water and wastewater utilities in the Pacific should perform or have, which could provide a useful framework to guide how utilities might make improvements (inputs), and thus complementing the performance benchmarking that measures outputs and outcomes. Scott et al. (2003) recommend ways to improve utility service delivery including to (i) implement strategies to utilize appropriate methods and technologies for water supply and sanitation systems and approaches; (ii) promote utility collaboration and regional partnership to reduce UFW to improve the sustainability of utilities and reduce the need for developing new water resources; (iii) create a sustainable environment for public and private investment by developing and implementing national, sector, and strategic plans that identify the economic, environmental, and social costs of different services and developing pricing policies to ensure proper allocation of resources; (iv) establish financially viable enterprises for WSS that result in improved performance by developing appropriate financial and costrecovery policies, tariffs, billing and collection systems, and financial and operating systems; (v) reduce costs through improved operational efficiency, using benchmarking, development of water loss reduction 
programs, and improved work practices; (vi) develop pro-poor policies that include tariffs with lifeline blocks and transparent and targeted subsidies.

Specifically relating to services to informal settlements, the following recommendations have been made. Central government authorities should establish clear national mandates for service delivery, clarify the organizational authority and their obligations to implement services, and set clear service level targets for settlements. Stakeholders should incorporate settlements into existing or emerging sector investment plans. Performance monitoring and evaluation should be associated with payment-based performance incentives. Partnerships should be explored between the local government or the water and sewerage utility and nongovernmental agencies (NGOs) or the private sector. (Schrecongost and Wong 2015)

Development partners should constructively support settlement inclusion through the provision of technical assistance and analysis in key areas that may not yet be a priority for government or be within the operating scope of utilities. These areas include cost benefit analysis of WSS investments; advocacy; policy development and strategic planning; research of technical options and piloting with NGOs and communitybased organizations; private sector support; peer-to-peer learning and exposure to international experience; and innovative financing. (Schrecongost and Wong 2015)

Utilities and other service providers need to accommodate locally driven exchanges of services and products to reach every household in an informal settlement (Barrington et al. 2017). For example, utilities typically provide only one way to access water and wastewater: command-based services, in which money is exchanged. They do not allow other exchanges to take place, such as on-selling of water from one connection to multiple households. Greater access would be achieved if utilities relaxed rules that discourage alternative exchange types (e.g., disallowing on-selling water), and supporting alternative exchange types would further increase coverage (e.g., charging bulk water tariffs for households that on-sell to multiple households, rather than for those households paying higher price or volume because they are a high water-using household (Saunders and Souter, in prep).

Mechanisms that support participatory and collective planning and action among enabling actors with informal settlements can lead to improved WASH situation governance to improve service delivery. Barrington et al. (2017) describes a model of participatory governance focused on urban and peri-urban informal settlements in Melanesia in which an informal forum of informal settlers, government, utility, and other enabling actors regularly meet to identify collective actions to improve WASH situations for informal settlements. In this model, external agencies and organizations are not limited to their formal roles and responsibilities, but rather, these actors are able to provide broader technical and nontechnical support and advice. (Barrington et al. 2017)

\subsubsection{Rural Water Services}

Community-managed water systems remain the dominant system for water supply in rural communities throughout Pacific Island countries. This rapid review does not identify any significant dialogue among Pacific stakeholders regarding an evolution of community-managed water systems to service delivery models, apart from some discussion for Papua New Guinea as part of its service delivery assessment.

Globally, there has been a shift away from demand-responsive community management, which has been the dominant approach for rural water supply in low-income countries (LICS) for the past two decades (Moriarty et al. 2013). There is growing acceptance that the community management approach, which is based upon informality and voluntarism, cannot provide the quality and reliability of services aligned with the rising expectations of users, their governments, or the global community. The shift is toward a service delivery approach, which emphasizes the entire life cycle of a service, including the hardware (engineering or construction elements) and software required to provide a certain level of access to water (Lockwood and Smits 2011). Service refers to the provision of a public benefit through a continuous and permanent flow of 
activities and resources. This concept has been applied to many other services-both in LICs and uppermiddle-income countries (UMICs) - such as health, education, electricity, telephone, and urban water supplies.

To support that outcome, there is a shift toward adoption of a wider range of service delivery models than community management alone. Governments have begun addressing the sustainable financing of all costs with a particular focus on external financing of capital maintenance (asset management) and direct support costs (Moriarty et al. 2013). Such service delivery models would comprise locally appropriate combinations of local service providers (including community-managed systems), intermediate service authorities (to provide planning, coordination, regulation and oversight, and technical assistance), and national policy and normative functioning (e.g., sector policies, regulatory frameworks, defined service levels, macro financial planning, and coordination of development partners). The emerging WASH resilience approach (UNICEF 2017b) recognizes the role of governments and NGOs in supporting communities to manage systems, although service delivery models (different mechanisms by which that might be achieved) are not discussed.

\subsection{Gaps in Knowledge}

\subsubsection{Service Delivery Models Suited to Pacific SIDS}

Apart from coverage statistics regarding access to major categories of access to infrastructure and facilities, there are limited data describing the common service delivery models for urban and rural populations of Pacific SIDS (i.e., consideration of life cycles and complete service chains for WSS, including fecal sludge management). Analyzing existing services using a service delivery framework would identify gaps in existing service arrangements and potentially identify successful models. Evaluating the type, scale, and mechanisms of technical support provided to community-managed systems (as described in chapter 8) might further identify models that are likely to be sustainable and scalable.

To encourage financing of urban and rural service delivery models, including consideration of public-private partnerships (PPPs), the economic, environmental, and social costs of different services needed to be incorporated to policies and pricing models. Participatory modeling approaches to assess water source and delivery options, such as that trialed in Kiribati (Moglia et al. 2008), could improve decision making.

Research regarding the applicability of rural WSS service delivery models outside the Pacific might identify additional service delivery arrangements. Outside the Pacific Island countries, there is currently much attention on drawing on lessons from international experiences (World Bank 2017) to identify models that might then be modified to suit local contexts. Modification needs to consider the issues of isolation and remoteness, diseconomies of scale, lower human capacity and rapidly transitioning communities, changing as a consequence of rapid urbanization, and increasing environmental variability.

\subsubsection{Analysis of Bottlenecks to Improvements to Service Delivery}

Although there will likely be similar bottlenecks to improvements to service delivery among some Pacific Island countries, the value of the service delivery assessment approach is the awareness and ownership gained by stakeholders through the participatory processes use, rather than the assessment results per se. Conducting service delivery assessments in clusters of Pacific Island countries with similar contexts may provide an economy-of-scale. In addition, it may provide an opportunity for cross-country sharing of challenges and opportunities for collaboratively (or cost-efficiently) progressing some bottlenecks.

\subsubsection{Engaging Urban Communities}


There are few mechanisms to engage urban communities, particularly informal settlements, in service delivery planning and implementation (e.g., Barrington et al. [2017] describe a general participatory approach). In some Pacific SIDS there is a clear need to build social cohesion before engagement with urban communities would be effective or constructive. For communities with moderate social cohesion, participatory mechanisms of engagement trialed elsewhere internationally could be useful, such as social contracts between utilities, government, and local communities to support tariff acceptance.

There is limited documentation or analyses of approaches, such as pro-poor strategies, being used by utilities, governments, or other enabling actors to engage with impoverished, marginalized urban communities (e.g., informal settlements). A review of existing strategies would identify successful mechanisms or critical gaps. There has been no attention paid to behavior change of impoverished, marginalized urban communities relating to willingness to pay for WSS services.

\subsubsection{Engaging Rural Communities and Water Users in Service Delivery}

A number of resources describe approaches to engage rural communities in WSS service delivery (Crennan 2004; UNICEF 2017a). It is not clear whether and how these different resources can and should be used concurrently. (Chapter 8 identifies related ways forward and knowledge gaps.) Underlying these approaches is an assumption that both communities and government, NGO, or other facilitators working with them have sufficient technical knowledge about water systems (environmental and social) to guide useful communitymanaged systems. As discussed in chapter 4 , existing capacity is not clear.

Engaging other major water users in rural service delivery planning and implementation may prove beneficial. Participatory approaches that support constructive engagement between all local stakeholders need to be identified. Some do exist, such as water stewardship, but they have not yet been widely used in the Pacific. 


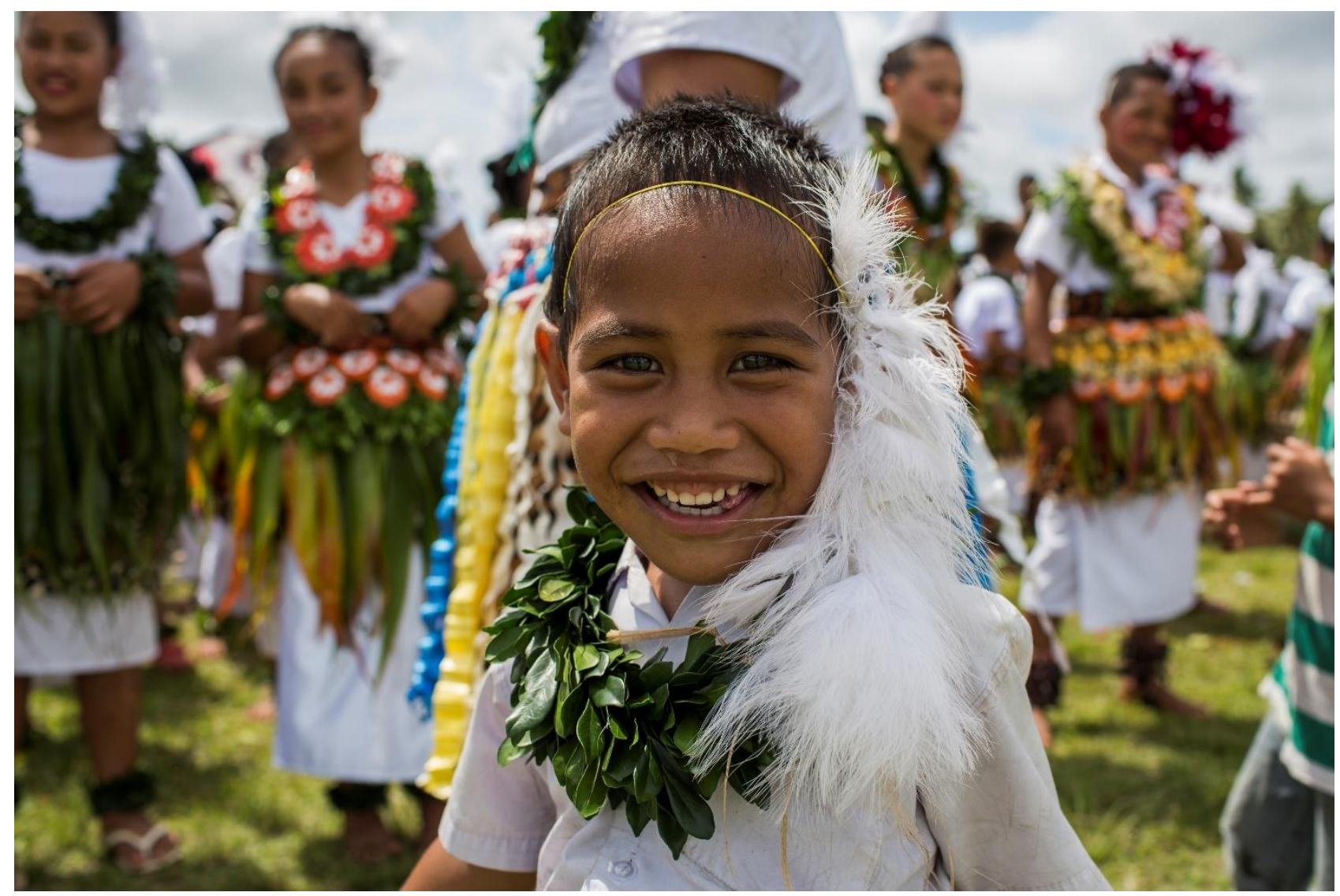

Festival in Tonga (Credit: World Bank)

\section{Sociocultural Aspects of Water}

\subsection{Nature of the Challenge}

Sociocultural factors in the Pacific region are critically important when considering any form of management or governance improvement. Water resource management is affected by (Carpenter and Jones 2004) the following:

- Traditional forms of governance in urban and rural areas, based on traditional sociocultural structures

- Role of land tenure and attitudes toward land among "traditional owners," which is embedded in the local community systems and structures

- General attitude to change and external influences, in which local commitment and the perception of "ownership" of projects and proposals are crucial if progress is to be made and sustained

- Relatively recent independence of most Pacific Island countries means that they are attempting to establish national identities apart from their history of the dominance of external cultural and organizational forms inherited from the colonial era

Carpenter and Jones (2004) conclude that the most important social issue for integrated water resources management (IWRM) and water governance is the need to ensure that water projects and management measures are designed and implemented consultatively so clear understandings are negotiated with those 
who are affected or need to participate. If solutions are designed without respect for traditional cultural attitudes and social structures, commitment will not be obtained and long-term success and sustainability are unlikely. A lack of such cooperation or understanding of the prevailing sociocultural order have characterized many projects in the past. Such issues can also be a problem for officials of central government agencies in their relationship with regional and rural communities. (Carpenter and Jones 2004)

During World Bank community-driven development (CDD) projects, small island nations have needed to be treated differently to recognize the complexity of not only a postconflict, low-income, low-capacity country but also a country composed of isolated islands with high costs and complex logistics. Lessons from traditional CDDs, such as in Indonesia, are difficult to transfer to the Solomon Islands. (World Bank 2016)

In addition to sociocultural issues affecting sustainable water management, tensions between the demands of urbanized societies and the traditional values, customs, and rights of subsistence communities, which have developed over millennia, are critical factors (Jones 1997; White et al. 1999). Some cultural factors may be used to advance the process; others may need to be managed so that they do not hinder the process; and some may display features that both help and hinder the process (Dworsky 2002). One example of a social institution that may constrain the anticipated project is the Polynesian family system, which can make demands of politicians and public officials that conflict with the requirements of their formal, national roles (Dworsky 2002). However, that same system may be a source of useful social support for those entrenched in the old way of doing business (Dworsky 2002).

\subsubsection{Water Resources and Customary Rights}

Water management and land ownership are inextricably linked, and in many Pacific Island countries, land tenure has customary rights including ownership of groundwater (White et al. 2006). The question of water ownership is a significant "stumbling block to the adoption of water policy in many Pacific Small Islands" and the idea of vesting water ownership in the state is at odds with traditional practices (White et al. 2006). In such situations, protection of groundwater sources by communities is essential.

Resolving land tenure issues and balancing traditional customary rights to land with those of the public interest are recurrent themes at the heart of many attempts to improve urban management and land planning throughout the Pacific. The failure to deal directly with land tenure and traditional organizations has caused projects to fail. Land tenure is relevant to water resource management and water governance because traditional ideas about land tenure and family and community rights can create complexity when it comes to identifying the right to take, use, and manage water. If the complex issues surrounding land and water rights are not properly resolved, water initiatives will not succeed. Therefore, dealing with land and its underlying sociocultural norms and values are an integral part of dealing with the governance of IWRM. (Carpenter and Jones 2004)

\section{Box 6.1 Water Governance Program in Pacific SIDS}

Supported by the Secretariat of the Pacific Community (SPC) and the European Union (EU), the Program for Water Governance (PfWG) aims to mainstream the principles of good water management into day to day applications and pilot projects to assist in achieving sustainable water resource management and provision of water services. PfWG recognizes the importance of institutional changes to support the transition from subsistence cultures to developing urban communities. Three pilot programs have been conducted in the late 2000s in the Solomon Islands, Fiji, and Kiribati, with a focus on improving national water resource management legal framework, policies, and strategies, or, at a local level, promoting awareness and water supply schemes management capacity.

Sources: PWA n.d. 
Some Pacific Island countries have atoll islands that are reliant upon groundwater as the main freshwater resource and those resources are threatened by human settlements. As a result, those governments have declared water reserves over major groundwater sources. Water reserves prohibit settlement and allow eviction of existing dwellers and land owners from the reserves. An example is in the atoll of South Tarawa in Kiribati (White et al. 2006). The declaration of water reserves over privately-owned land causes considerable tensions and conflict between affected communities and the government because of the loss of local amenity when land owners are evicted from their land or proscribed from traditional land uses. This has led to costly ongoing disputes and vandalism of water infrastructure and groundwater monitoring boreholes (White et al. 1999). Wider communities regard designated water reserves as common property whose resources can be plundered. Squatting, market gardening, and pig raising also occur. To reduce conflict, the government pays affected landowners annual commercial rents for the land resumed as water reserves. However, it has been suggested that governments pay reserve landowners to be custodians of reserves and manage their protection, rather than pay rentals or compensation for groundwater reserves (Jones 2001; White et al. 1999).

\subsubsection{Working with Communities}

The sociocultural factors outlined previously emphasize the fundamental reasons governments and other actors need to work with communities, however it appears this has been variably successful in Pacific small island developing states (SIDS). Chapter 5 summarizes analytical knowledge relating to community-managed systems. Apart from playing a role in delivering a service, communities might be involved in water management in a range of ways, not least in the planning, design, and implementation of local water and sanitation systems.

Ownership of project outputs by the communities they serve is made difficult by the requirement of some donors, funders, and implementing organizations for labeling infrastructure outputs with information telling the reader of its provenance. These identifying labels generally make no mention of the contribution made by the local community, nor do they contain any reference to the community in which they are located. They are generic reminders of the largesse of others, and emphasize the community's dependency. (Clarke, Feeny, and Donnelly 2014).

Strengths-based approaches to community development are one way of operationalizing participatory development principles. They are based on the understanding that working with communities to recognize strengths is more likely to inspire positive action rather than a focus on needs and problems. The Solomon Islands NGO Partnership Agreement (SINPA) supports strengths-based approaches implemented by nongovernmental organizations (NGOs) from 2009-14. Strengths-based approaches are considered "new" when used as in a country in which externally driven development support has led to citizens and communities becoming clients of aid, rather than active citizens. However, stakeholders consider the approach to resonate well with understood roles, responsibilities, and structures in terms of the Solomon Islands' kastom. The features include adherence to beliefs about innate community capacity, the need to draw on community resources to create change, and the delicate balance between a focus on strengths and repression of problems that might need to be surfaced. However, the NGOs diverged from the idealized strengths-based approach in that they saw the development process as a partnership between themselves and communities rather than community-led (Willetts et al. 2014).

Willetts et al. (2014) concludes that strengths-based approach takes significant time and is not conducive to short-term interventions; even a five-year program is insufficient to instill and embed a new way of approaching development and to reach extensive tangible development outcomes. It also cannot be considered an antidote to the complexities of working with varying motivations, power relations, interests, and skills within communities. Ways to address and negotiate these challenges are needed, including consideration of the dynamics likely to ensue after an NGO leaves. 
The demonstrated intangible outcomes of increased confidence, self-esteem, motivation, and hope bode well for stimulating the ongoing generative processes referred to in the strengths-based approach literature. Such outcomes are critical for facilitating the Solomon Islander-led development conceived of in SINPA's original design and for operationalizing participatory development principles more broadly.

\subsubsection{Culture, Gender, and Community-Managed Systems}

Pacific women in rural areas are often responsible for household drinking water management, sanitation, and health promotion. As such, women have often accumulated considerable knowledge on water source location, water quality, and storage methods. However, government efforts geared toward improving the management of water resources and ensuring access to safe drinking water and adequate sanitation often overlook the central role of women (WHO 2016).

Community leadership is critical to successful community initiatives. In the Melanesian culture, status is very important and gained in many ways apart from formal leadership, unlike a chiefly system of governance, in which the "big man" system of local governance exists (Clarke, Feeny, and Donnelly 2014). It is not uncommon for men to be very enthusiastic during the implementation phase of projects, in particular when there is infrastructure involved. To be seen to be bringing, and contributing to, the largesse to the community brings status. However, there appears to be little status to be gained from subsequent maintenance of the products of largesse, such as water systems. Water and hygiene are generally women's responsibility in Melanesian societies. Men are keen to establish infrastructure for the status they derive from doing so. However, there is no status for men in ensuring that women have easy access to water because women will gather and carry water regardless of where it is sourced. Ongoing public monitoring, however, can provide an opportunity for men to obtain regular public attention and status from the demonstration of their abilities and expertise in organization and management of a maintained system. If the monitoring is done by outsiders there are no local and public opportunities for men to be acknowledged.

\subsubsection{Gender and WASH}

In informal settlements of Papua New Guinea, women and girls are at serious risk from poor water supply, sanitation, and hygiene (WASH) services. Collecting water at night or using a toilet far from the house exposes women to sexual and physical violence; even intrahousehold discussions about the need to have a toilet or better water supply can lead to domestic violence. Women bear the heavy physical and time-consuming burden of collecting water. Despite women having primary responsibility for every household task using water and cleaning toilets, they have no say in decisions about improving water supply and sanitation (WSS) services. Anecdotally, these observations are common to rural contexts, as well as other Pacific SIDS (at least Melanesian). (WSP 2014)

WHO (2016) reports that selected communities in Vanuatu have identified far-reaching gender equality outcomes from their community WASH projects. Women report positive changes in gender relations in their families, households, and communities, and greater inclusion of women in community decision-making processes beyond WASH issues. Women also report a reduction in violence at the household level and are able to reduce their labor in collecting water and meet their own needs for WASH facilities, saving them time and improving their health and dignity (WHO 2016). Gender assessments have been undertaken in Kiribati, the Solomon Islands, and Vanuatu: increased knowledge on the topic have led to legislation changes in Vanuatu's water resources bill (Mommen, Humphries-Waa, and Gwavuya 2017)

Gender outcomes that have been attributed to WASH initiatives encompass those directly related to improved services as well as outcomes that move into areas of relationships, power, and status (Carrard et al. 2013). Carrard et al. (2013) identify many outcomes including those occurring within individuals and with their relationships with others; and those occurring at varying scales from household and family networks, local social and community networks, and broader governance institutions. That research provides a useful 
detailed list of the many gender-related changes that might result from WASH interventions, both positive and negative. Authors acknowledge that WASH programs can entrench or exacerbate gender inequities.

\subsubsection{Political Stability, Will, Corruption, and Social Institutions}

Political commitment to and ownership of water management and reform agendas are essential. However, reform is a destabilizing process, and no government undertakes such a process without some risk of becoming so unpopular that it loses its mandate to rule. Thus, much courage, patience, and commitment on the part of government are needed. Political stability is a significant factor influencing water and wastewater development projects. Regular political changes indicate the need for governments to be far more inclusive and participatory in their reform initiatives, thus ensuring that the momentum of reform continues despite changes in government. Past political upheavals (e.g., in Vanuatu, Fiji, and the Solomon Islands) point to the need for governments to be more sensitive and responsive to growing inequities between segments of society. The volatility of political changes in the Pacific appears endemic and must considered when working with governments to plan and implement reform and development strategies. (Dworsky 2002)

In some Pacific SIDS, there are risks of corruption by politicians or bureaucrats that could undermine investments in water management. For example, in the Solomon Islands, although the government has made sincere efforts to counter corruption and misappropriation in recent years, serious capacity shortfalls and premature loading of government WASH functional agencies by donor-driven programs of assistance risk tolerating a high degree of management weakness. Civil society organizations are gaining confidence to publicly challenge the government on its fiduciary control record, with potentially important consequences for both the political economy and social order. (Maskul 2016)

Underlying political stability, corruption, and misappropriation are the traditional social institutions that require obligations and commitments to one's tribe and community (e.g., wantok in Papua New Guinea and the Solomon Islands; kerekere in Fiji; bubuti in Kiribati). These obligations can lead to challenges with the systematic and equitable provision of services. For example, there has been evidence of correlation between tribes and kin and corruption, suggesting that officials may be favoring their own ethnic groups (Lamour 1997).

These social institutions also have social safety net benefits. Where these structures remain strong they are central to social cohesion (DFAT 2012 [). Some of the benefits of such social cohesion include (ILO 2006) (i) sustaining those in need of basic necessities; (ii) sharing and distributing "surplus" goods (thus using resources efficiently); (iii) ensuring community self-sufficiency and sustenance; and (iv) maintaining social solidarity and mutual relations within kin groups and communities. Mane (2016) has suggested that the wantok system in the Solomon Islands can be used to facilitate development, considering its influence on family and social cohesion, as well as on land ownership.

\subsubsection{Attitudes to Informal Settlements}

The rapid urbanization of Pacific towns includes rapid growth of informal settlements (Jones 2012b) whose water and waste situations are typically very poor. This is likely because of (i) the exclusion of informal settlers from formal service delivery mechanisms (see chapter 5 ), and (ii) a tendency for informal settlements to exist as urban villages with rural-like attitudes to services (Jones 2012b). Sociocultural factors, in part, are likely to contribute to the exclusion of informal settlers from formal service provision. These settlements are generally unwelcomed by urban residents and governments. The reasons are not well-articulated in the water-related literature reviewed here, but likely to be a complex of sociocultural (especially ethnic) factors as well as structural issues (e.g., the increased demand by larger populations on employment, services, and infrastructure). 


\subsubsection{Culture and WASH Behaviors}

\section{Water Use Behaviors}

Per capita consumption of freshwater in most small island countries is increasing. Past designs for freshwater reticulation systems in atolls have used 30-50 liters per capita per day as the demand estimate; growing expectations in larger villages suggest 100-150 liters per capita per day is a more reasonable estimate. Some reticulation systems are unable to meet demands (especially because leakage is typically significant); therefore, water is supplied for only a few hours per day as de facto demand management. Because of uncertainty of supply, people leave taps open to intercept the supply. This greatly increases losses. Urban communities therefore often supplement water supplies using domestic wells and small rainwater tanks, but during droughts these are quickly exhausted or contaminated. Metering and charging for water supplies, a long-recognized demand management tool, is successful in some small islands. Where lack of resources is significant, there is reluctance to introduce metering or it has been abandoned in favor of flat fees, containing no conservation message. (White et al. 2007)

Some rural and urban residents are required to carry water from afar and store water at their houses. These practices are not always conducted safely and may cause contamination of otherwise safe drinking water, such as in informal settlements of Papua New Guinea (WSP 2014). One Kiribati study (Psutka et al. 2013) finds that because of poor practices relating to storing water, handwashing and sanitation, the introduction of a household water filter to reduce exposure to contaminated water would unlikely improve water quality.

\section{Sanitation Practices}

Joint Monitoring Programme (JMP) statistics summarizing main types of sanitation offer an insight to attitudes and practices around sanitation (given that sanitation practices are most influenced by individual, household, and community norms and priorities, rather than structural barriers). Thirteen percent of the population practice open defecation across Pacific Island countries; the 35 percent of the population use improved or shared sanitation indicates a third are willing to invest in sanitation. The diversity within the region is largely aligned with cultures-Melanesians displaying the lowest rates of investment in sanitationfurther indicating the importance of cultural factors on sanitation behaviors. A Kiribati study finds that 34 percent of respondents, and 57 percent of their children, practice open defecation, and that handwashing at important times is uncommon (Psutka et al. 2013).

The role of beliefs and customs in sanitation programs in the Pacific is documented by Paul (1958. He identifies three problems with programs seeking to change sanitation behaviors that are still relevant today. First, there is a tendency to assume that locals have odd beliefs and habits, and that they are captives of blind custom, while program designers are relatively free from cultural peculiarities. There is failure to recognize that measured by the standards of one culture, the manifestations of another are bound to appear more or less arbitrary or bizarre, and may be, labeled as superstitions or uncouth custom.

Second, there is the assumption that "our" ways and ideas are more advanced than "theirs." Some aspects of culture, notably scientific knowledge and technical skills, are indeed subject to measurement and relative ranking. But knowledge is not wisdom, and many aspects of culture, including language, esthetics, moral codes, and religious values, lie beyond objective rating for want of a culture-free standard of measurement. It is a mistake and an insult to intentionally or inadvertently imply that because some areas of the world are technically underdeveloped their people or their cultures are in general underdeveloped.

Finally, there is a tendency to view customs and beliefs as isolated elements rather than as parts of a system or pattern. The connections are not always apparent, but often people cling to a particular practice or belief not merely because it is familiar and traditional but because it is linked to other elements of the culture. Conversely, a change in one area of the culture may bring with it unexpected changes in other areas or may result in awkward dislocations. 
The adoption of unfamiliar technologies has met challenges. Ecosanitation (composting toilets) have been trialed in several Pacific Island countries in the past 20 years. Lessons from early trials in Kiribati and Tongaand some limited use in Fiji, the Federated States of Micronesia, and Palau-include (i) importance of locally constructed and "owned" composting toilets; (ii) leadership of women in ecosanitation projects (local and expatriate women); (iii) importance of direct experience of the toilets; (iv) effectiveness of linking to noneducational motivators to change sanitation behaviors; and ( $v$ ) demonstrations of how to take holistic approaches to WASH in practice. (Crennan and Berry 2002)

A recent focus has been trials in Tuvalu as part of the Global Environment Facility (GEF) IWRM project managed by SPC, in which 40 compost toilets were installed, following promotion of the technology and application from interested residents. A results report by the project team (Seleganiu 2013) notes significant challenges in gaining acceptance of the new approach to sanitation, and the cost (US\$1,000 to US\$2,500 per unit, covered principally by donors). The approach is being spread to other atoll nations. This rapid review is unable to find evaluations or research exploring the specific challenges and strategies to addressing sociocultural constrains to ecosanitation, nor of the uptake and adoption of ecosanitation beyond the project boundary.

\section{Hygiene Practices}

In Papua New Guinea, most households understand how germs spread from feces to hands to mouths, and the importance of washing hands to avoid diarrhea, but rarely is handwashing practiced (WSP 2014). Proper hygiene behavior, in particular handwashing with soap, is rarely practiced at household level in rural areas of the Solomon Islands, though there are encouraging signs of gradual hygiene behavior shifts in places where school-centered WASH activities have been targeted and related product markets are allowed to expand (Marskall 2016). Unpublished studies of knowledge, attitudes, and practices indicates this is true of several Pacific Island countries (e.g., Live \& Learn Environment Education organization, Melanesia).

Among Papua New Guineans, Bukenya and Nwokolo reported in 1990 widely held beliefs that infant feces is safe and little is done to dispose safely of infant feces. Combined with the high rate of diarrhea indicating pathogenic infection, this belief creates a high risk to public health (Kamundi et al., n.d.). Anecdotally this belief appears to be widespread among at least some Melanesian cultures.

\section{Social and Religious Influences on Agency and Investment in WASH}

At least in some cultures, people's capacity to invest in WASH is constrained by sociocultural factors. The adaptive capacity of people, which determines their opportunities to identify and secure water and waste management solutions, is defined by "the set of resources (natural, financial, institutional or human, and including access to information, expertise, and social networks) available for adaptation, as well as the ability or capacity of that system to use these resources effectively in the pursuit of adaptation," (Kuruppu 2012). In Kiribati, the adaptive capacity of people is compromised by religious and cultural factors. Significant proportions of household financial resources are spent on maintaining social identity, especially through the church; thus, fewer resources are available for pursuing multiple water sources to provide sustained access (Kuruppu 2009). In addition, church power structures constrain benefits to the individual, depriving people of their freedom to exercise autonomous agency and achieve personal well-being. Anecdotal information indicates this is commonplace in Melanesian communities, too. In contrast, community approaches to total sanitation (CLTS) in Kiribati and the Solomon Islands have linked with faith-based organizations to advance sanitation discussions in communities, using references to the Bible. This evidence indicates that approaches intending to strengthen adaptive capacity, which are sometimes include in participatory approaches, may be limited by the challenges faced by Pacific islanders in exercising agency. 


\subsection{Ways Forward}

Genuine participation by community members in all aspects of designing, planning, and managing water resources and services have emerged regularly from the literature as the best way forward to adequately take into account, and leverage, sociocultural aspects of Pacific SIDS (Crennan 2002; Dworsky 2002; White et al. 2007b). Also recommended is acceptance that such participatory approaches will require long-term investments (Willetts et al. 2014).

Recommendations relating to engaging rural and informal communities in service delivery roles are summarized in chapter 5 . This chapter paper focuses on additional recommendations relating to acknowledging and working within sociocultural factors relating to water management.

\subsubsection{Customary Rights to Water}

Regarding government or other actors wanting to extract and use groundwater from customary land rather than pay rentals or compensation for groundwater reserves, one recommendation is that governments should pay reserve landowners to be custodians of reserves and manage their protection, (Jones 2001; White et al. 1999) to ensure local land owners retain an active role in managing their land and water. In addition, reference is made to a number of approaches to address and manage conflicts between local communities with rights over water reserves and their governments, such as SPC's Program on Water Governance (PoWG), although these approaches have not always been well elaborated. One example is a conflict resolution and negotiation support strategy that uses a role-playing, which has been trialed in Kiribati (Perez et al. 2003).

\subsubsection{Engaging with Communities on their Services}

Most water-related projects have a water management committee as a key output in the project design; however, few are functional beyond the end of project. Leadership by women increased the likelihood of a functional committee. Functioning water systems are more likely when the control and maintenance is the result of a strong leader-male or female-with a vested interest in the maintenance of the system. When implementing projects, true leaders need to be involved, and in many instances, they are women. (Clarke, Feeny, and Donnelly 2014)

Ongoing monitoring by communities of their water and waste systems can provide an opportunity for men to obtain regular public attention and status from the demonstration of their abilities and expertise in organization and management of a maintained system. If the monitoring is done by outsiders there are no local and public opportunities for men to be acknowledged. (Clarke, Feeny, and Donnelly 2014)

For rural populations in Pacific SIDS, there are several approaches that have been applied successfully. Crennan (2004) provides guidance for field workers working with rural communities, and identifies a set of guiding questions to engage communities in planning and managing water systems, rather than an overly prescriptive approach applicable only to a narrow content. These guidelines specifically consider issues of gender equity, poverty alleviation, and water, in an integrated way. The water safety planning approach and its variations (e.g., UNICEF's WASH resilience), when done well, are also opportunities for participatory water planning and management with rural communities.

The guidance for engaging urban communities is more limited. Barrington et al. (2017) provides specific approaches that government utilities or NGOs can use to engage with informal settlements, and collectively plan and manage WASH. These approaches are based upon participatory community development and research, and the capabilities approach. 


\subsubsection{Gender}

Halcrow et al. (2010) has developed a guidebook for practitioners in Pacific Island countries. The authors recommend that addressing gender and WASH be achieved by (i) promoting positive and empowering relationships between program staff and the community and between members of the community; (ii) seeing and valuing the different contributions and views of women and men, to further their community's development; (iii) ensuring that women and men can meaningfully contribute to discussions and decisionmaking relating to WASH in their community; (iv) promoting WASH outcomes that address the different but equally important needs and hopes of women and men; and ( $v$ ) recognizing that WASH programs are not only a pathway to better water access and quality, sanitation, and hygiene but can also promote positive and respectful roles, responsibilities, and relationships between women and women, women and men, and men and men within communities.

Carrard et al. (2013) have developed a conceptual framework for classifying gender equality changes arising from WASH programs to assist practitioners and researchers in planning, identifying, and documenting gender outcomes. In addition, IWRM advocates mainstreaming gender into the program with recommendations: (i) commission project-specific gender analysis and gender action plans; (ii) strengthen gender-disaggregated data collection including qualitative and quantitative data; (iii) encourage the adoption of gender champions within national committees; (iv) support gender training and capacity building at all levels; and (v) ensure the continuous provision of gender inputs (WHO 2016).

The Asian Development Bank (ADB) has prioritized gender equality as a driver of change in its 2020 Strategy. A gender checklist on WSS has been developed to provide guidance at all stages of ADB's program cycle in determining access to resources, roles, and responsibilities; constraints; and priorities. It also aids and in designing appropriate gender-sensitive strategies, components, and indicators to respond to gender issues. (WHO 2016)

In Tonga, efforts to strengthen the role of women in the provision of domestic drinking water through rainwater harvesting have resulted in guidelines and a specific participatory manual used by the Tonga Community Development Trust to support communities (WHO 2016). Through the Village Women's Development Program, village operation and maintenance funds are managed by women to strengthen the sustainability of rainwater harvesting systems.

\subsubsection{Behavior Change}

Most attempts to influence local behaviors appear to be either education-based (e.g., promotion of particular practices because they are good for the health or people or the environment), or through participatory approaches, such as participatory rural appraisal, and participatory learning and action. An example of participatory learning and action has been its application to improving pig waste management with rural communities in Fiji (Terry and Khatri 2009). Facilitators wished to identify and understand factors that influenced two communities in implementing changes to current pig waste management practices. The focus area was the Coral Coast in the south of Viti Levu Island, where international tourism is expanding but stream and coastal waters are under threat of pollution from the largely uncontrolled release of pig waste. The investigation finds that participatory approaches are effective at the village level if correct tools are used and if the research process adapts to community needs. In particular, participatory learning and action methods in the two study villages (Komave and Votua) assisted demographic groups to identify and then prioritize a range of pig waste problems, including deteriorating water quality, public health, and agricultural sustainability. Encouragement by the facilitators for communities to take ownership of these problems led eventually to one village installing a new pig management system. This has become a showcase as a successful local rural development project with long-term benefits for the local environment and the continuing growth of tourism in the area. 
A structural-based approach to demand management is being trialed in Kiribati. Small, single dwelling 500liter storage tanks are filled continually from the reticulation system by a slow trickle feed, sufficient for a family's daily demand. Conservation of water then becomes a household responsibility. Behavioral change is fundamental for conserving and protecting water, and education and awareness programs have increased, particularly in the Pacific. (White et al. 2007b)

Within Pacific SIDS there is limited evidence of investment into modern approaches to behavior change (i.e., those based not exclusively on education and awareness strategies). Modern approaches identify a full range of barriers to behaviors and utilizes education as well as emotional-based messages to encourage improved WASH behaviors.

We are aware of some modern approaches that have not yet appeared in the literature. International Water Centre (IWC) has been developing evidence-based behavior change campaigns, such as handwashing with soap with rural coastal and island communities in Papua New Guinea (with United Church); and infant feces management with rural villages (with WaterAid); and menstrual hygiene management in schools (with WaterAid). Other organizations are experimenting with nontraditional behavior promotion, including Live \& Learn Environment Education organization (handwashing with informal settlements) and UNICEF Solomon Islands (handwashing).

CLTS has been formally adopted as the sanitation promotion approach in the Solomon Islands, and is used in some other Pacific SIDS. CLTS is based almost entirely on emotional triggers to change sanitation behaviors. There is little evidence about the impact (positive and negative) of these approaches. Some interventions are relatively recent and monitoring is yet to occur; some have been implemented for some time (e.g., CLTS in the Solomon Islands) with no indication in documents of any plans to evaluate these interventions.

\subsection{Gaps in Knowledge}

Despite recognizing the importance of considering and integrating social and cultural aspects to water activities, and accepting that participatory approaches are therefore critical, there is little research to systematically or rigorously evaluate trialed approaches or develop new approaches is apparent; exceptions include capabilities approach (Kuruppu 2012) and strengths-based approach (Willetts et al. 2014). Understanding the range of specific participatory approaches that have proven successful (or not) in Pacific SIDS would increase the likelihood and efficiency of positive outcomes and minimize negative outcomes from water investments.

\subsubsection{Addressing Customary Land and Water Rights}

Relating to customary land and water ownership, there is the recommendation to pay custom owners to manage and protect groundwater (White et al. 2007b). This review does not identify research that elaborates on this idea, or the general concept of payment for ecosystem services. Payment for ecosystems services involves paying landowners a regular income to protect environmental services that have a value to the whole society, such as groundwater and healthy catchments. This concept might provide a mechanism to secure groundwater resources as well as maintaining the active involvement of landowners (with some responsibility for good quality water resources in return for income). Further research seems necessary to explore the receptiveness of landowners, governments, and utilities to the approach of payments for ecosystem services, and to identify and test the effectiveness of a range of arrangements.

A similar concept could be applicable to nonrural settings, such as for informal settlements that live over or near to raw water resources that supply urban water systems and where sanitation services are nonexistent. Paying local residents to provide those services (e.g., solid waste collection or fecal sludge management) could contribute to reduced water treatment costs by utilities, and reduced costs to the health system. 


\subsubsection{Leveraging Traditional Social Institutions}

Although identified as a potentially useful opportunity to improve development generally, the strategies to achieve this in regard to water management are not evident in the literature.

\subsubsection{Gender and Water}

Although some attention has been directed toward identifying the gender-related outcomes associated with WASH projects, there is little beyond generalized statements of gender empowerment relating to women's involvement in other water-related activities. In addition, regarding WASH, gaps remain in understanding (Carrard et al. 2013) (i) the applicability of reported outcomes to urban areas, and to local, provincial, and national governance scales; (ii) gender outcomes at different stages of life, such as outcomes for adolescent and younger girls (e.g., relating to menstrual hygiene and school attendance), older women, pregnant women, or female heads of households; (iii) changes experienced by men and boys.

\section{Addressing Behaviors and Attitudes}

Approaches to address the nonacceptance of urban-rural migrants, which is necessary if WASH service delivery and improved health and wellbeing of these urban residents is to be achieved, are not identified in the water-related literature reviewed here. A more thorough review of broader literature, such as social and urbanization literature, might identify approaches that could be applied to WASH service delivery efforts. Similarly, strategies to address the attitudes of informal settlers to urban services, in particular a willingness to pay for water and waste services, are not identified in the literature and seem a likely gap in knowledge. Other gaps relating to behavior change seem apparent, including (i) the effectiveness of existing campaigns for handwashing with soap, infant feces management, and CLTS; (ii) applicability beyond the local contexts in which they have been developed or applied; and (iii) water use efficiency and safe carting and storing of water. 


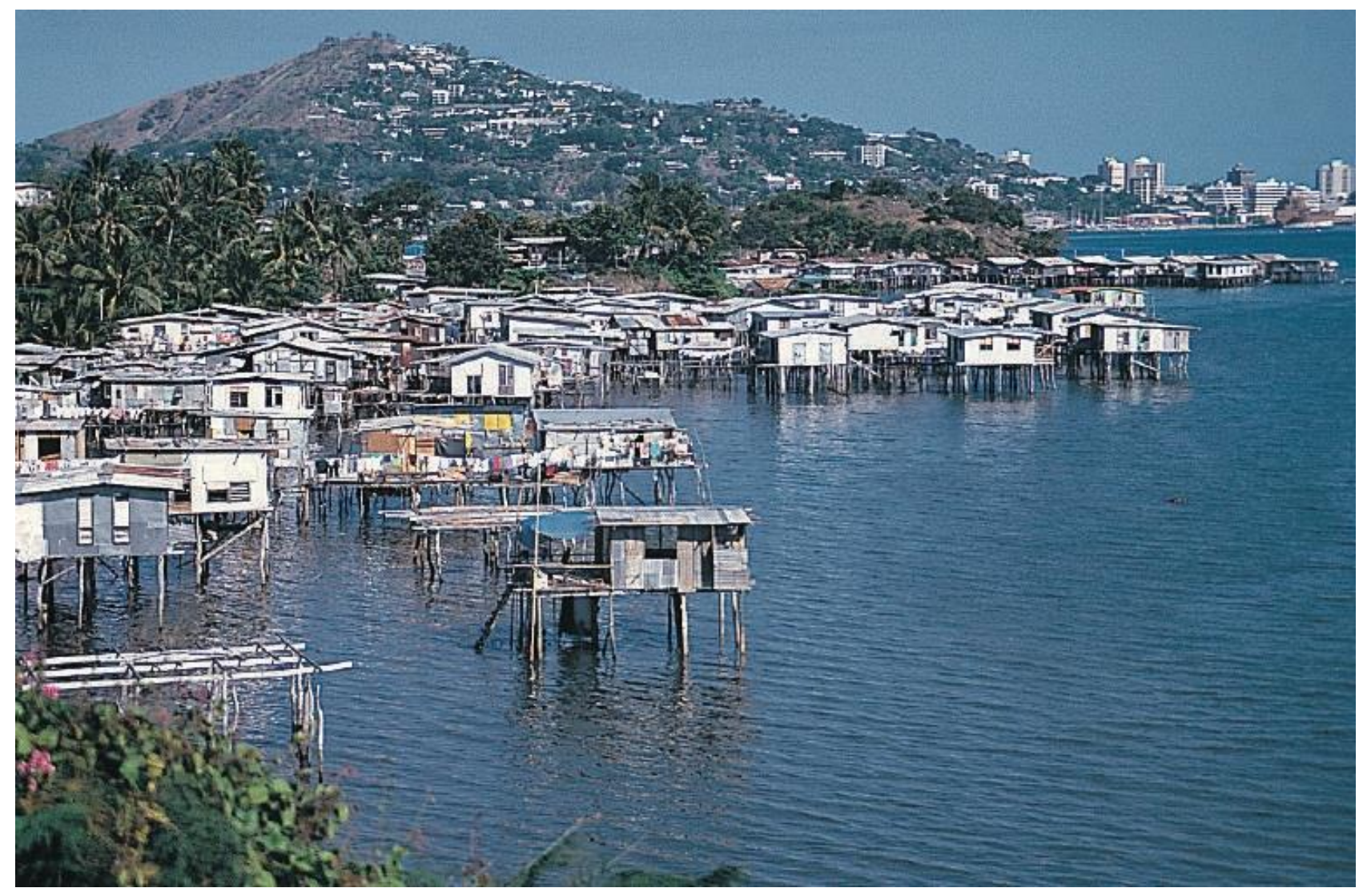

(Credit: World Bank)

\section{Unplanned Urbanization and Water}

\subsection{Nature of the Challenge}

At current population growth rates, the urban population of the Pacific small island developing states (SIDS) is expected to double over the coming 25 years. Urban populations in Kiribati, the Solomon Islands, and Vanuatu are expected to rise significantly. In the short term, the most significant increases in urban population growth are expected in Melanesia, followed by the Federated States of Micronesia (ADB 2012a).

\section{Box 7.1 Non-Pacific SIDS-Globally, Urbanization in SIDS Less Important than in Pacific SIDS}

Of the 65 million people living in SIDS today, 38 million (59 percent) already live in urban settlements. Singapore and Nauru are among the most urbanized SIDS (100 percent), while Trinidad and Tobago (13 percent) and Papua New Guinea (12 percent) are among the least. The urbanization rate among SIDS from 2010-15 represented 1.4 percent per annum, just below the global average of 1.7 percent per annum, but with striking regional and national differences. The Pacific region is the highest with 4.3 percent urbanization rate. Global urbanization trends are commonly coupled with an increase in gross domestic product (GDP), especially in urban areas, through expanded services and a matured industrial sector. However, in the case of some SIDS, urbanization has not brought the expected formal increase in GDP. This can be attributed to the fact that these economies remain driven by the informal sector, agriculture, and tourism.

Sources: UN Habitat 2015 
Urbanization in the Pacific displays diverse patterns and is geographically uneven. It is largely fueled by rural to urban migration by people seeking better opportunities to improve their quality of life, such as through access to economic growth opportunities and employment. However, the socioeconomic benefits of residing in urban areas are not evenly distributed. Urban poor populations can struggle to gain access to housing, land, infrastructure, and basic services, or to participate in political decision making (ADB 2012a). Furthermore, the "primacy of capitals" is a distinct feature in SIDS. Many capitals continue to be the primary hub of administrative and economic activity, resulting in a skewed concentration of labor, political systems, and administrative services. The concentration of human settlements along with economic and social activities at or near the coast is also a well-documented feature of small islands, making them more vulnerable to climate variability and disasters (UN Habitat 2015). Urbanization in Pacific Island countries present many challenges for water management and water security relating to land use planning and tenure, governance, utilities, provision of water supply and sanitation (WSS) services, and impacts on human and environmental health.

\subsubsection{Land Use Planning and Tenure}

There are often limited stocks of state land for urban development and a lack of affordable, reasonable housing delivered through the formal urban planning mechanisms. As a result, housing for most of the growing Pacific urban population is through traditional land tenure agreements or informal agreements and built independently of official planning systems (WHO 2015; WSP 2014). The greatest number and highest concentration of informal and unplanned settlements in the Pacific are in Melanesia (ABD 2012).

Traditional and modern urban planning systems generally exist side by side in Pacific urban areas and there is often limited clarity regarding the role and mandate of government. This makes it difficult for governments to secure areas for new urban development (WHO 2015). In some areas, such as Port Moresby's informal settlements, town plans and policies are effectively irrelevant as they are not implemented or compatible with land management practices at a community level (Jones 2011).

Increasing ad hoc growth based in informal and squatter settlements includes development on land that is not always

\section{Photo 7.1: Informal settlements on steep sloping lands in Honiara}

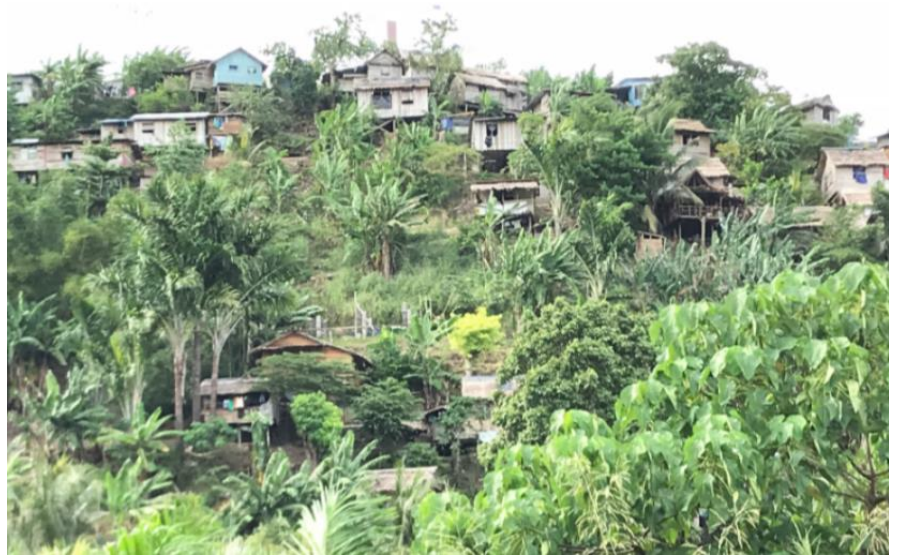

Credit: Stephane Dahan/World Bank suitable for development - river banks (Apia and Suva), steep sloping lands (land adjoining Port Moresby's CBD, Honiara as shown on Photo 7.1), flood prone lands (Apia, Nuku'alofa, South Tarawa and Suva), exposed coastlines, and mangroves. Poorly managed urban development in these hazard vulnerable environments places pressure on surface water quality, and creates human health risks through the spread of communicable and water-borne diseases (ABD 2012; WHO 2015; WSP 2015). 


\subsubsection{Governance}

Governance aspects of urban planning and provision of WSS services in the Pacific can present significant challenges. Rural-urban migration can bring a diversity of people together (WSP 2014), sometimes resulting in conflicting cultural values and practices. Conflict can also arise concerning land ownership and security of tenure, forced evictions (ADB 2012a), and use of WSS resources (WSP 2014). Issues of fairness in decision making at the community level have hindered the provision of WASH services in some areas (WSP 2015).

Governments have often been slow to respond to a growing number of urban residents who are increasingly unsatisfied with their quality of life (ADB 2012a). Government bodies may be reluctant to address issues of urban planning and urban poverty due to financial costs of action, sociocultural issues, and landowner issues stemming from the mobilization of customary or traditional lands (Jones and Lea 2007).

Despite a substantial need, political commitment to ensure WASH service access in Melanesian informal settlements is often weak (WSP 2015). Government decision makers in Melanesia often do not make connections between WASH services and urban public and environmental health (WSP 2015). Where there is political will to serve informal settlements, challenges from policy environments and financial and organizational capacity can present obstacles (Schrecongost and Wong 2015).

\subsubsection{Utilities and Provision of WSS Services}

Urban areas struggle to respond to increasing demand for water supply and sanitation services fueled by population growth. Across the Pacific there are great disparities in access to urban water supply systems. Utilities in Melanesia often have no commercial incentive to extend water supply to informal settlements, and in some cases, they face legal and technical barriers (Schrecongost and Wong 2015; WSP 2015). Additionally, Melanesian urban water utilities tend to focus on traditional piped sewer systems to the exclusion of other nonpiped systems. This leads to the exclusion of informal settlements from sanitation services, because they are more suited to nonpiped solutions (WSP 2015). Utility water may be contaminated at the point of use due to informal storage systems and low-quality shared connections (WSP 2015). Water supply systems often are subject to large pipeline losses (Falkland 2011).

For some households (such as much of Port Moresby, Papua New Guinea), water may be available for a cost, either sold by a utility or illegally provided through an intermediary (Jones 2011; WSP 2015). Financial, land tenure, or cultural obstacles can restrict households from accessing WSS services even when they are provided (Schrecongost and Wong 2015; WSP 2015). Where utility water supply services are not accessible or consistent, households use surface water sources, accessible groundwater supplies, or in some cases collected rainwater. Surface water and groundwater supplies tend to be contaminated due to a lack of sanitation systems (WSP 2015).

Capital expenditure for water and wastewater services in the Pacific is often funded through donor grants and loans, but maintenance expenditure generally is insufficient to support assets in the long term. Very few water utilities recover enough revenue to provide a commercial return. When utilities or informal providers of water address a community's immediate water demands, complementary services such as drainage or wastewater treatment are usually not established. Drainage systems in many Pacific urban areas need repair or reconstruction, contributing to flood risks (Poustie and Deletic, 2012). This can generate disease risks where there is increased standing water for disease vectors or fecal pathogens (Jones 2012b); WSP 2015).

\section{Box 7.2 Non-Pacific SIDS-Low Efficiency, and Lack of Customer Orientation of Caribbean Urban Utilities}

All utilities in the Caribbean are public; only those in the Bahamas, Belize, Jamaica, Barbados, the Cayman Islands, and Antigua and Barbuda have performance contracts with their line ministries. Cash flows barely cover operating expenses for many utilities: in 10 out of 18 utilities, operational costs exceed revenues being generated by the services provided. Timeline of latest tariff increases vary from 1995 (Antigua) to 2013 (Jamaica, Belize, St. Lucia). Unaccounted-for water (UFW) across the Caribbean region ranges from 17 percent 
to 66 percent. Energy use and labor costs remain high. Several Caribbean utilities have clear vision and mission statements and customer charters. Most either don't or don't publicize them. Many utilities have weak websites or communications activities. Caribbean utility managers also mentioned the need to avoid micromanagement by their board of directors (who serve for short period and have little water experience). They identified the following areas for further regional initiatives and collaboration (Berg 2014):

- Data collection and benchmarking (related to key performance indicators)

- Financial sustainability (reducing dependence on periodic infusions of government funds)

- Citizen charters (meaningful and well-publicized mission statements)

- Capacity building (promotion of professional development and team work)

- Workshops for boards of directors (as part of an emphasis on governance in incentives)

- Web pages and greater transparency (internal and external communication)

- Water safety plans and utility resilience (techniques for risk recognition and mitigation)

- Institutional collaboration (workshops for interagency cooperation)

- World Bank Institute (WBI) eLearning (access to toolkits and other material on water and wastewater)

- Other eLearning platforms (formats for continuing education and sharing of expertise)

Melanesian WASH projects that have targeted informal settlements have tended to be uncoordinated (without connection to the wider urban fabric), and serve a small number of households. None have addressed citywide fecal sludge management, and in places in which improved toilets are constructed it is rare for waste to be safely removed, transported, treated, or reused (Schrecongost and Wong 2015). Nongovernmental organizations (NGOs) and civil society organizations have tended to advocate for either water supply, sanitation, and hygiene (WASH) investments in rural areas or large-scale piped infrastructure projects in formal areas that yield limited benefit to poor communities (WSP 2015).

\subsubsection{Impacts on Human and Environmental Health}

Urban areas can hold high risks of contraction of infectious and noncommunicable diseases in large part because of poor sanitation facilities, poor water quality, poor land quality, and population densities that facilitate spread of infection (ADB 2012a). A lack of on-site retention systems, reticulated sewerage systems, and limited stormwater treatment means that urban wastewater often drains directly to surface waters or groundwater. In many areas, leaking septic tanks contribute to groundwater and marine contamination. Urban waterborne pollutants (such as those from industrial discharge, petrochemicals, detergents, pesticides, heavy metals, and untreated sewage) pose water quality and public health problems.

\subsubsection{Anticipated Interactions and Impacts on Water Management}

Further growth in informal and peri-urban settlements, characterized by formal unemployment and low standards of living, is expected (ADB 2012a; Storey 2005). This poses challenges for the provision of safe WSS, protection of water quality, and water demand management (Falkland 2011).

In Melanesia, urban population size, urban security concerns, scale of squatter and informal settlements, access to land, political instability, ethnic tensions, and degradation of the urban environment suggest that its urban challenges are more complex and diverse than those elsewhere in the Pacific (ADB 2012a). Ongoing long-term commitment will be required from governments and NGOs to foster working partnerships between different communities, utilities, and government at local and national levels (WSP 2015).

\section{Box 7.3 Non-Pacific SIDS-Water Safety Planning in Caribbean Utilities}

Water safety planning is an area of concern for utility managers in the Caribbean but very few do it: "In those settings where there is no regulator, water safety plans do not seem to be high priority for water ministries or other oversight agencies." (Berg 2014). General environmental degradation in urban area calls for an integrated approach 
to water and environmental management. A UN-Habitat study finds that ecosystem-based approaches to adaptation are more cost-effective than pure engineering options in protecting coastal areas.

Sources: Berg 2014; Nurse and McLean 2014; UN Habitat 2015

In urban and peri-urban coral atoll communities, water supply coverage is expected to decrease due to high population growth rates matched with limited capabilities of water supply and sanitation systems (White et al. 2004). Governance aspects of water management and planning in urban areas may require concerted long-term efforts and may present ongoing significant challenges (Shrecongost and Wong 2015).

\subsection{Ways Forward}

Ways forward for providing water supply and sanitation to rapidly urbanizing areas in the Pacific include (i) coordinated national government action and policy, (ii) coordinated city-region planning, integrated water resources management (IWRM) and water sensitive approaches, attention to conflict resolution, institutional and financing arrangements, and appropriate technology.

\subsubsection{Coordinated National Government Action and Policy}

Urban planning and urban management need to take a higher priority on national agendas. This would include policies relating to land provision, shelter, and essential services such as WSS (ADB 2012a). Integrated and prioritized policy at national and city levels and effective institutional arrangements are crucial in fostering a much-needed coordinated approach to urban water projects and investment. Ad-hoc projects and fragmented approaches have proved to be insufficient (Storey 1998).

\subsubsection{Coordinated City-Region Planning}

There is a need for projects that promote community scale infrastructure and services that are integrated with urban planning and management at a city and town scale (including peri-urban areas), and that promote an IWRM framework (Hadwen et al. 2015). There is a need for greater emphasis on WASH investment in informal settlements, especially in Melanesian countries. However, investment should not focus on hygiene aspects without corresponding efforts to enhance accessibility to water supply and sanitation at a broader community level, and should link with wider city-level management and planning (WSP 2015).

\subsubsection{IWRM, Water Sensitive Approaches, and Appropriate Technology}

It has been argued that traditional centralized infrastructure alone is not sufficient to address wastewater and stormwater challenges in Pacific urban areas (Poustie and Deletic 2014). In industrialized countries, new paradigms of IWRM are emerging in recognition of the failures and limitations of traditional approaches to managing water and urban landscapes. Water system developments in Pacific urban areas should seek more advanced approaches to water management (such as those found in IWRM and "water-sensitive cities" approaches) (Poustie and Deletic 2014). This may require efforts to build capacity of urban and water authorities and utilities in Pacific Island cities. It has been recommended that NGO projects work to increase provision of water supply and sanitation services and investigate innovative designs or approaches to services

\footnotetext{
${ }^{5}$ Water sensitive cities are said to be underpinned by the following principles for sustainable urban water management: using diverse water sources through both centralized and decentralized infrastructure; providing ecosystem services for the natural and built environment; and enhancing sociopolitical capacity for sustainable management of and behaviors toward water (Brown, Rogers, and Werbeloff 2016; Wong and Brown 2009).The term water sensitive cities is generally used not to describe actual cities, but as a vision and set of principles for cities to work toward (Brown, Rogers, and Werbeloff 2016).
} 
and infrastructure, and that they collaborate with partners in government. Such collaborations are needed to increase institutional capacity, especially in Melanesian countries (WSP 2015).

There have been efforts to support the development of a range of appropriate and low-cost on-site sanitation options that can gain community acceptance and that can be built and maintained by residents of informal urban settlements (WSP 2014). Other interests in innovative appropriate technologies include the mooted use of pebble matrix and handmade clay balls for filtration and treatment of highly turbid water during heavy rainfall events (Rajapaske and Fenner 2011; Rajapaske et al. 2012). The Sanitation Park project in Fiji, funded by the New Zealand Agency for International Development and the World Health Organization (WHO), sought to support communities in identifying and solving their sanitation problems by examining and selecting from a variety of appropriate affordable wastewater treatment options located in a demonstration park (Bower et al. 2005).

Social and cultural acceptance of on-site sanitation solutions can vary. Community perceptions and values relating to aspects and types of sanitation need to be considered. Chapter 6 discusses issues relating to cultural acceptance of nonconventional sanitation solutions.

\subsubsection{Attention to Conflict Resolution}

Given the potential for conflict and equity dimensions of urbanization in the Pacific, resolving tensions between state and customary land management, and forging new ways of thinking about informal and squatter settlements are required (Storey 1998). Effective urban governance is needed (WHO 2015) that can allocate financial resources to manage and deliver services, raise revenue, set legal and regulatory arrangements to ensure acceptable land use patterns, and facilitate both formal and informal decisions on land use and land development (ADB 2012a).

It is recommended that urban management arrangements are tailored to the characteristics and requirements of individual countries and settlements, and that they address longstanding cultural traditions while ensuring public accountability (ADB 2012a; Jones and Lea 2007). This in turn requires new approaches to local participation and institutional alignment of municipal authorities, ethnic and cultural groups, and community leaders (Jones 2012b; Storey 2003).

\subsubsection{Institutional and Financing Arrangements}

Utility investments are influenced by donor and government technical preferences that have tended to favor investments in large piped water infrastructure servicing formal settlements. Institutional and financing arrangements should be reviewed to identify opportunities to increase the financial feasibility of extending services to informal settlements (Schrecongost and Wong 2015). For example, establishing equity measures in performance monitoring and payment arrangements for water utilities may help to generate greater financial incentives for utilities to extend services to poorer urban communities. Also, establishing comprehensive national sanitation policy and enforcement arrangements can further ingrain equity measures in service provision (WSP 2015). Authorities should set clear mandates for service delivery, clarify roles and responsibilities, and establish clear service delivery targets for urban settlements (Schrecongost and Wong 2015).

Although there are cases of government intervention to increase water supply to informal settlements - such as community service obligations administered to the water utility Edu Ranu in Papua New Guinea requiring the installation of community taps in informal settlements-limited transparency, coordination, monitoring, and regulation can lead to suboptimal outcomes. NGOs working to increase provision of water supply and sanitation services can collaborate with partners in government to increase institutional capacity and foster transparent and coordinated processes in planning for water access and sanitation for informal settlements. This may be particularly beneficial in Melanesian countries. (WSP 2015). Investigations into financing options 
for improved sanitation, such as low interest loans, may also yield benefits for informal settlements (World Bank 2014b).

\section{Box 7.4 Case Studies in Pacific Island Countries}

\section{Planning and Urban Management Agency (PUMA), Samoa}

Efforts to develop inclusive planning models in Apia, Samoa, have included the establishment of a Planning and Urban Management Agency (PUMA) by the Samoan government. PUMA was established as part of efforts to develop institutional arrangements for more holistic management and planning of urban and peri-urban areas. It was founded on extensive consultation and the integration of customary practices as well as government responsibilities (Storey 2003). PUMA has responsibilities for developing plans and policies, regulating development, coordinating urban services, and disaster management (Storey 2005).

The Vaitele Urban Governance Pilot Project 2008-12 was jointly funded by the government of Samoa and UNDP, and managed by the Planning and Management Agency (PUMA). The project piloted new forms of governance in three non-traditional peri-urban villages located on freehold land. They trialed the appointment of Mayors within each village, reflecting features of traditional governance (ADB 2012a).

\section{Urban Development Program for South Tarawa, Kiribati}

The South Tarawa Urban Development Program (formerly the Sustainable Towns Program) was an urban sector review and support program undertaken by the New Zealand Aid Program and based on a joint New Zealand Aid Program and AusAID-funded project design carried out in 2007. It sought a sectorwide approach to urban development and management and included projects to provide residents in Betio and Bairiki villages with greater access to on-site sanitation facilities and potable water, installation of rainwater tanks, efforts to enhance awareness of personal hygiene, and establish mechanisms for maintenance and operation of facilities. Additionally, the project aimed to subdivide and provide 150 serviced residential plots for low-to middle-income families on state land (ADB 2012a). This was started following an overall assessment of wastewater reticulation, treatment, and disposal alternatives (Fraser Thomas Partners 2011).

\section{Cities Development Initiative for Asia (CDIA), Fiji}

Established by the Asian Development Bank (ADB) and the German government, this initiative has provided assistance to cities in Asia and the Pacific to identify and develop urban investment projects that correspond with city development plans while providing good governance, pro-poor development, environmental sustainability, and climate change adaptation. Four councils in the Greater Suva area engaged with this initiative to address deteriorating infrastructure resulting from rapid population growth in squatter and informal settlements. The project included the development of a well-planned, well-serviced urban corridor. CDIA was asked to produce an investment program for infrastructure that focused on wastewater management, flood protection, drainage, and solid waste management. CDIA prepared feasibility studies for potential infrastructure financing (ADB 2012s). Five project outputs were improved drainage and stormwater management, protected riverbanks, improved river or stream flood management, strengthened sector capacity for planning and management, and strengthened capacity for project implementation. As a part of this, a council rating system was extended to informal settlements as a pilot (CDIA 2012).

\subsection{Gaps in Knowledge}

There are limited data on informal settlements in Melanesia (Schrecongost and Wong 2015), and on periurban growth and development (Storey 2005). More information is needed regarding the scale and nature of challenges affecting WASH in informal settlements in individual Pacific countries. This includes trend data relating to population growth, health, service access, and incomes. Existing data are limited (WSP 2015). More information is needed on peri-urban areas to allow for proactive anticipatory urban planning that is cognizant of how quickly urban landscapes are changing and growing (WSP 2015). 
There is little literature relating to the identification of WSS systems (service delivery models, infrastructure and governance arrangements) suited to communities undergoing transition (e.g., villages transitioning to small urban centers; small urban centers expanding to larger urban centers, especially with expanding periurban and informal settlements; or urban centers needing to transition to water sensitive futures). There appears to be a gap in understanding which urban analysis and planning approaches would allow for adaptive and evolving infrastructure and service delivery models as communities continue to change. Research is needed on fecal sludge in urban areas, including opportunities for fecal desludging of pit latrines, and for reuse of fecal sludge (World Bank 2014b). 


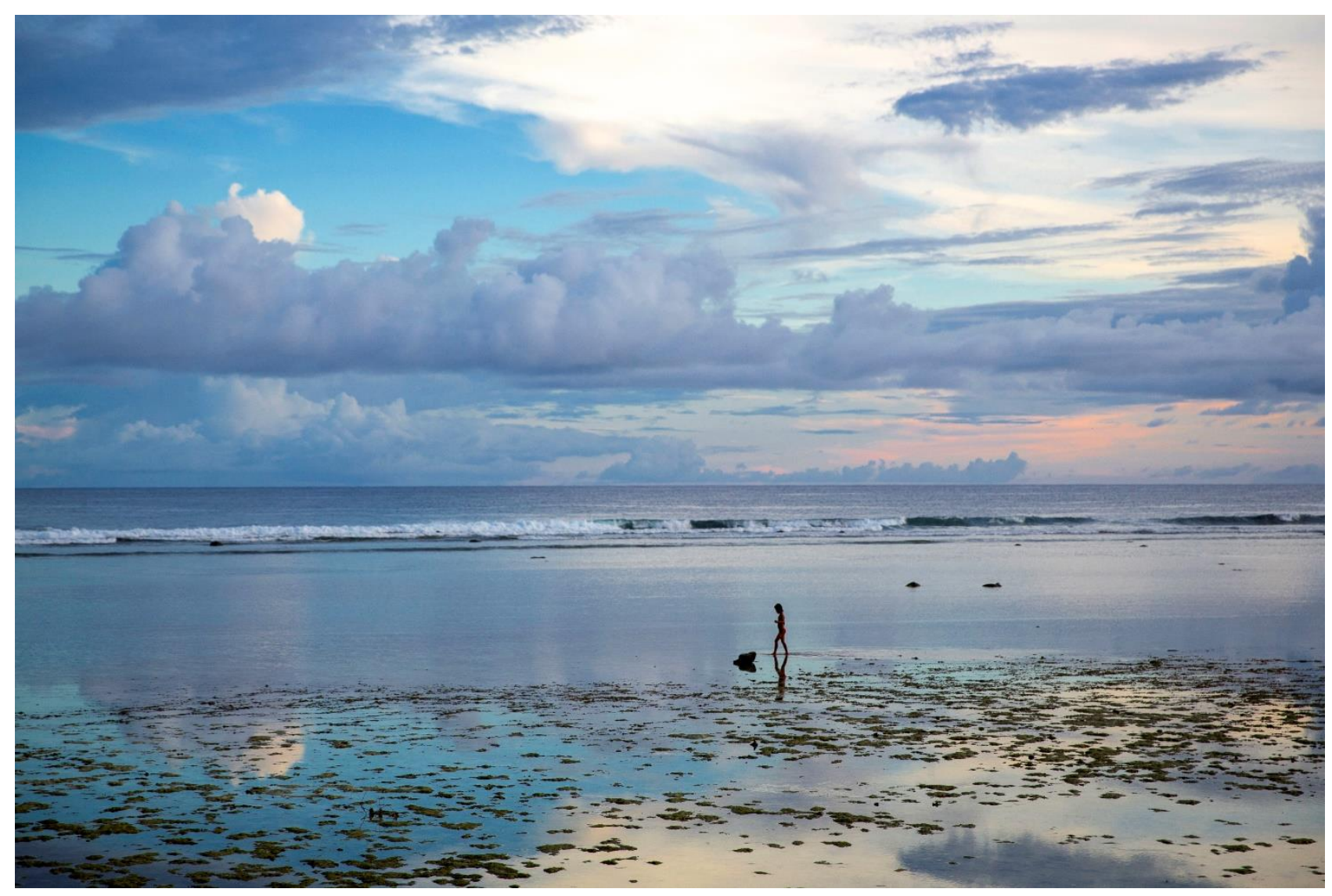

Child walking along the beach in Bikenibeu village, South Tarawa, Kiribati (Credit: World Bank)

\section{Small and Remote Populations and Water}

\subsection{Nature of the Challenge}

The small and remote Pacific Islands belong to a larger group of countries that the economist Paul Collier called "the bottom billion" in 2007. This group-which also includes countries in Central Africa, Central Asia and Caucasus, East Africa, and West Africa-has fallen behind in the global hierarchy of nations as countries are often trapped in conflict, suffer from a natural resources curse, are small with poor governance, and are landlocked with bad neighbors or otherwise isolated from the rest of the world (WDR 2009).

Becker (IMF 2012) shows that small island states in the Pacific are typically different from countries in other regional groupings in that they are extremely geographically isolated and have limited scope to tap into economies of scale due to small populations. Of the small countries in the Pacific, almost all are so small that scope to access economies of scale would likely be a significant issue. Only Fiji's gross national income (GNI) is larger than the median income of small states, a measure of the potential opportunity to reap economies of scale.

The remoteness of small Pacific islands is a distinguishing characteristic, not only in terms of distance from the nearest continent but also from neighboring countries. As an example, Samoa, the Marshall Islands, Tuvalu, Kiribati, and Tonga are some of the most isolated states in the world; each is more than 3,000 kilometers from the nearest continent, Australia (Becker 2012). While technological progress has allowed 
countries to overcome some of these barriers, such as those related to effective communication, distance remains a key challenge to overcome when physical factors are important. (Becker 2012).

The impacts of these issues on water services and management can be summarized as follows. Typically, small populations of Pacific SIDS are correlated with total incomes (and tax bases) that are small, and the fixed costs associated with the provision of public goods and services have to be spread across a very narrow base. As a result, fixed costs may represent an unusually high share of national income. One consequence might be that there is insufficient tax revenue to secure the public provision of basic health services, transportation, or government administration. These constraints subsequently often result in small administrations that lack the capacity to function efficiently. The degree to which this is an issue varies between countries. (Becker 2012)

These higher costs per capita may be exacerbated by the vulnerability of many Pacific SIDS to natural disasters, this requiring higher cgost infrastructure that is more resilient to disasters (Becker 2012). Access to, and cost of, supply (distribution) chains are reduced for water supply and sanitation (WSS) infrastructure due to the cost of transport and small purchasing power. The transport costs associated with trade and commerce are higher as distance increases (Becker 2012), and the minimal economies of scale are improved by infrequent services. Sharing of infrastructure with upper-middle-income countries (UMICs) is difficult. Basic infrastructure may need to be duplicated if populations are dispersed across countries comprising groups of islands (Becker 2012). Deploying skilled workers is costly and slow due to the frequency and cost of transport to remote areas, which are low due to small market demand. This impacts abilities to (i) provide onsite technical support and skills development for regular operation and maintenance and community planning and (ii) respond rapidly following a disaster.

Developing locally based skilled workers is difficult. The capacity for Pacific SID governments (and their education and training institutions) to offer quality education and training for water-related skills is low, and even fewer training opportunities for remote and small populations (ADB 2008). Due to isolation, there is limited access to skilled labor markets and high costs for labor mobility to transport people. Pacific SIDS are typically considerable distances away from the major labor markets that are better able to supply a more skilled workforce, such as in Australia and New Zealand (Becker 2012).

Genuine community participation is costly. The difficulty in deploying skilled workers and minimal opportunities for locally based training and the requirement for long-term engagement for successful community participation prevent significant challenges to achieving effective and genuine community participation in water management with remote communities.

There is constrained ability to prepare for disasters and responses are slower: maximum resilience to disasters requires communities having immediate access to disaster response equipment and skilled disaster responders (among other factors). The dispersed nature of small communities means the cost to duplicate and transport equipment and people is high. Carefully planning for disasters involving geographically spreading workers and equipment to local hubs (e.g., island towns) either permanently or in response to disaster warnings reduces the impact to slower and ineffective disaster responses.

There is also limited access to cost-effective and timely supply chains (to market) for any industries or businesses. Furthermore, they are usually not on routes of major shipping lanes that connect major producers with markets and are therefore unlikely candidates for establishing trade hubs. In outer islands, the combination of limited access to markets, with reduced water security and infrastructure, means industries such as large-scale agriculture are weak. 
Photo 8.1: Villagers in the remote Torricelli Mountains in West Sepik, Papua New Guinea transporting water tanks to their community

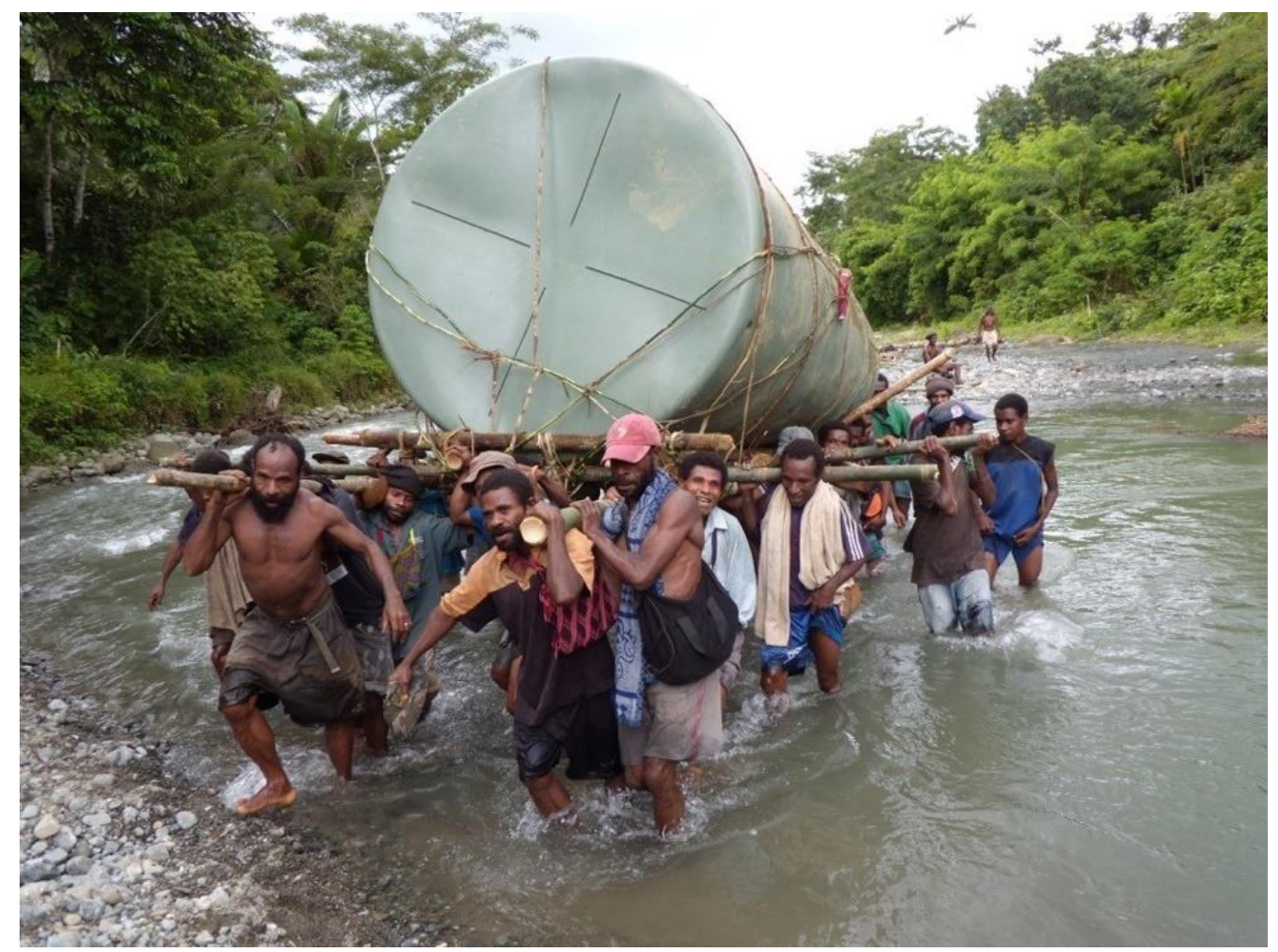

Credit: Tenkile Conservation Foundation, Lumi

\subsection{Lessons from Other Global Regions with Remote and Small Populations}

\subsubsection{Multicountry Evaluations of Rural Water Systems}

The recent World Bank (2017) multicountry review of rural water supply systems contains recommendations relevant to remote and dispersed populations; as such, it is potentially applicable to remote and small populations in Pacific SIDS. Supported self-supply systems seem to be the recommendation for such communities. These are systems in which households, or small clusters of households, provide their own solutions to water supply, and the approach is formally recognized by governments, which have adopted programs of structured support to accelerate and improve service delivery under this model.

Direct local government provision tends to perform poorly, especially in low capacity environments. Despite the various efforts to promote supported self-supply as a formal service delivery model-only present in Ethiopia - there is a remarkable lack of documentation, which would be critical to convince policy makers of the benefits of promoting this model for remote and dispersed communities.

Integrating rural areas under the expanding service areas of public utilities comes with challenges, such as mobilizing human resources for asset maintenance, and extending billing and collection services and monitoring to remote areas. In Morocco, Office National de l'Electricité et de l'Eau Potable (ONEE) is testing various approaches to address these challenges, including local micro-enterprises for asset maintenance and private sector partnerships for the management of village-level distribution networks, while ONEE retains control of the bulk water supply. 
While the policy position in many countries is full cost recovery through tariffs without distinguishing between urban or rural areas, it is recommended that this position should be revisited and nuanced. Precise language is thus needed in tariff policies and guidelines, and flexibility for cost recovery approaches and propoor measures is advisable for different rural contexts (e.g., remote villages versus rural small towns).

Because the speed and appetite for reform and sector capacity vary from a country to another, the pathway toward sustainability will likely best be achieved through incremental improvements, and at different paces across segments of the populations. A ladder of sector development for rural services outlines basic, intermediate, and advanced sectors. The review indicates that the pace of sector improvement will be slower for remote, dispersed populations, compared with rural villages and growth centers and peri-urban and small towns.

Hutchings et al. (2017) review a range of community-management models of rural water supply systems in India. They conclude that, to be effective and sustainable, all community-management models must include some external support, and outlined three general models: Orofessionalized community-based management; community management with direct support (community management PLUS); and direct provision with community involvement. They also conclude that there are some situations in which the provision of services cannot be sustained by the community, either because their access to financial and human resources is too low, or the cost and complexity of systems are necessarily high due to environmental constraints. In these situations, governments (and formalized service providers) should accept their social obligation to provide subsidized or free water services.

\subsubsection{Remote and Small Indigenous Populations in Australia}

Australian remote indigenous communities in the Northern Territory are serviced by the Power and Water Corp. (a government-owned corporation), through Indigenous Essential Services (IES), a not-for-profit subsidiary of PWC. This organization provides services to 20 small towns and 52 remote communities, with 100-3,000 people in each community, spread over 1.3 million square kilometers. Isolation, remoteness, and small economies of scale are a challenge, similar to that of Pacific SIDS, with the difference being the isolation is caused by large expanses of land rather than sea, such that overland transport is feasible (not likely faster than boats between islands, but easier to control frequency and cost).

In addition, water security is an ongoing concern, and natural disasters and other factors cause power outages (other challenges that are in common with Pacific SIDS). The Power and Water Corp. uses an integrated approach to planning, development, operations, and maintenance for power and WSS services, providing some economies of scale in transport and training of staff that can operate across these services.

In towns, systems similar to urban systems are installed and maintained. Small remote communities utilize a diversity of technologies, including ones at the household, communal, or community levels. Consideration is given to technologies and infrastructure that work together to mitigate risks from water, power, or waste outages. The service provider plays a significant role in disaster and emergency response. The service provider has technical capabilities organized by electrical services, mechanical services, water supply, sewerage systems, water quality, community water planning, renewable energy, community liaison (auditing), and planning.

Domestic clients pay for electricity through use of prepaid meters. However, they do not currently pay for water or sewerage services: commercial and government clients pay for all services. The service provider finances its services through some revenue, core funding from the provincial government, and project funding from the national government. The service satisfaction is high, indicating success of the model.

The key strategies underlying their ability to provide a high level of service to remote and small communities include significant operational and maintenance cost efficiencies with organizational technical expertise, and 
on-site operators (to service, monitor, and manage power and water systems). All operators are appropriately trained and inducted.

On-site operators, or local essential service operators (ESO), are located in the communities. They operate and maintain the supply systems through contracts with shires, councils, and private contractors. They are fundamental to the delivery of services on the ground and provide immediate responses. They are also provided a career path from trainee, to ESO to ESO supervisor, providing skills transferable to the community, utilities, and the mining sector. Mentoring and on-site skills development is effective.

The operators benefit from partnerships with educational and training institutions to provide skills development (accredited). There is resource planning, including cost-effective larger-scale contracts for larger quantities of resources (e.g., provincial or state and regional or multiple towns and communities), which provide economies of scale. There is a coordinated and prioritized program for the delivery of routine maintenance, minor upgrades, and capital projects. The staff culture is innovative and integrated, with collaboration between energy, water, and sewerage services that may not be available within separate utilities or agencies. This allows operational delivery and planning teams to work closely together on improving services.

The program can also leverage off other power and water corporation services. The system operates under a risk-based approach that integrates and prioritizes risks to service outcomes (health a safety, finance, environment, and service delivery) across water, waste, and power service chains. Communities are actively involved with the risk assessments, which presumably increase awareness of risk prevention and mitigation actions the community could take, as well as input to and awareness of relative priorities for future investment. There are also likely many relevant lessons from water sector management for remote and small communities in Africa, and a review of that knowledge base could be valuable.

\subsection{Lessons from other Sectors}

Health, education, and energy sectors have the most similarity to water and waste service delivery. These sectors all require local infrastructure with local capacity to provide a local service, and which are supported by centralized oversight. The telecommunications sector may also have valuable insights relevant to the water sector, but due to time and resource constraints, the knowledge relating to telecommunications are not included in this rapid review.

\subsubsection{Health}

The major challenge for the countries is the provision of service on an equitable basis to populations scattered over many islands. The wide dispersion and remote location of populations in the Pacific creates challenges to the delivery of health services (Jarris and Sellers 2017):

- Difficulties with keeping clinics and dispensaries adequately staffed and supplied (infrequent and expensive transport costs).

- Difficulty in establishing and sustaining specialized equipment in small communities lacking economies of scale (e.g., to address the rise in noncommunicable diseases such as cancers, ischemic heart disease, renal failure), requiring transferring of patients for treatment at great expense to the health system.

- Telecommunications for training and education is generally weak and unreliable (though improving in some areas).

- Chronic shortages of trained health workers. Sending students outside for training is expensive and often unsuccessful (due to weak academic skills, and subsequent outmigration of newly skilled workers). 
Suggested ways forward include (i) offering formal nursing and public health degrees through local community colleges to provide versatile generalists who can do most public health work competently; (ii) temporary training programs delivered in the region for needed health professions; (iii) embedding faculty from regional health professions into colleges and universities to deliver curricula with local health agencies; and (iv) distance learning platforms offering accredited university-level courses.

In contrast, there are some advantages arising from the isolation and small populations. For example, the less complex health services environment in rural communities enables integrated models of health care. Usually, at a community-scale, a single service provides all aspects of health services, making it easier to create integrated health information systems, allowing health practitioners to link several preventative and curative services and supporting integrated models of health care. In addition, traditional and church social structures can be leveraged to improve the uptake of preventative services (e.g., immunizations and followup clinical services). They may also implement health policies at a community scale. For example, in the Federated States of Micronesia, traditional chiefs in Pohnpei have outlawed serving soft drinks at traditional ceremonies.

Some of the specific strategies being implemented in health to address the challenge of sufficient skilled human resources in remote and small populations could be applied to the water sector. However, currently there appear to be very few water-related courses, accredited or not, offered within Pacific SIDS.

Pacific Open Learning Health Net (POLHN). Launched in 2003, it is a mechanism for supporting training and continuing professional development opportunities for geographically dispersed and remote health workers in the Pacific. The platform partners with academic institutions and course providers to build institutional capacity and provide greater access to range of accredited and nonaccredited, online and offline courses. Fifteen countries and 45 learning centers (health clinics) participate, currently supporting around 30,000 health workers. In addition, the POLHN sponsorship scheme provides full funding for 100 staff from Pacific ministries of health to take instructor-led postgraduate courses. These can be completed without students leaving their communities.

Pacific Human Resources for Health Alliance (PHRHA). It is a regional body dedicated to identifying and implementing strategic measures to strengthen human resources for health (HRH) capacities in the Pacific. It brings together national governments, international agencies, academic institutions, professional associations, and development partners.

Regulatory standards and competencies. The World Health Organization (WHO) has supported the development of standards and competencies in the Pacific, such as the Pacific Code of Practice (2007), which was developed in response to the increasing concern about the loss of skilled Pacific health workers to recruiting low-income countries (LICS) and a need to ensure that transparent, fair, and mutually beneficial recruitment strategies and management are in place and followed.

\subsubsection{Education}

Geographic isolation and small populations are one of the main factors constraining access to and the quality of primary and secondary education in Pacific SIDS (UNECSO 2015). There have been improvements to access, and these are attributed to commitment to the Pacific Education for All initiative by governments and society and by the international community; partnerships at all levels, including those between Pacific Islands Countries and Territories (PICTs) and development partners; regional cooperation through high-level forums and regional institutions; increasing demand for a qualified workforce; integrated national education planning and budgeting; school-based improvement initiatives; curriculum reform; and national policies for early childhood education.

Key lessons from the Pacific Education for All (EFA) review (UNESCO 2015) that are relevant to universal sustainable water management include (i) realistic planning; (ii) committing to a vision and plan; (iii) having 
a dedicated budget for education; (iv) investing in skilled professionals (teachers); (v) having accountability (of teacher, school, and ministry performance); (vi) monitoring and using performance data; (vii) maintaining and developing partnerships; and (vii) recognizing the lifelong nature of education (with regard to water, this would relate to recognizing the value of, and requirement for, life-long use of water resources).

With regard to partnerships in the context of limited financial and human resources of most Pacific Island countries, regional cooperation and partnerships with institutions such as the University of the South Pacific are vital enabling factors. Regional cooperation is particularly helpful with regard to teacher training, assessing and supporting the development of literacy and numeracy, and skills development.

\subsubsection{Energy}

In an attempt to expand access to electricity to populations spread across tens of thousands of islands, governments and development partners in Pacific SIDS continue to prioritize development of electricity grids, as is evident in ongoing subsidization of grid-based power consumption and the establishment of ambitious (grid-based) renewable energy targets. Dornan (2014) argues that these traditional approaches to rural electrification which prioritize grid extension, are not suited to the Pacific islands region. Increased funding should be directed by both governments and development partners toward rural electrification, especially in off-grid areas in which isolated systems are more appropriate. Institutional reform is also important. Regulatory reform is needed for power utilities to extend electricity grids into rural areas. Institutional arrangements that facilitate the sustainable operation and maintenance of off-grid systems need to be established. Past donor and government-funded off-grid rural electrification projects have rarely been sustainable. Alternative approaches involving payment of output-based subsidies to energy service companies are worth exploring, although will succeed only where sound regulatory arrangements are in place.

\subsection{Ways Forward for Water Management and Services for Remote and Small Populations}

This rapid review of Pacific SIDS water knowledge identifies few recommendations specific to achieving water management and WSS services in remote and small populations. The following are potential ways forward extrapolated from the lessons outlined previously.

\subsubsection{WSS Service Delivery to Outer Islands and Communities}

WSS service delivery models that do not rely on urban systems are generally more appropriate, and this is reflected in current investments in water systems to date (rural systems do not seek to link to or mimic urban systems). However, other lessons from the energy sector that could apply to WSS service delivery are worth noting. First, institutional arrangements that properly facilitate and support the sustainable operation and maintenance of rural systems need to be in place. As with energy, past donor and government-funded remote or rural water projects have rarely been sustainable due to a lack of ongoing technical and management support. This recommendation is in common with that of Hutchings et al. (2017) and the World Bank (2017), which both recommend all community-managed and self-supply systems require technical and resource support. In addition, output-based rewards for community service operators, and potentially local government support officers (regular financial or other rewards-based upon delivery of sufficient WSS services), with sufficient oversight, monitoring and regulatory arrangements, should be in place.

In reviewing lessons from the health sector, the observation that the community context provides excellent opportunity for integrated approaches is a useful reminder of the opportunity for synergies arising from integrating water-related services with others, such as waste and sanitation, but also natural resource 
management, public health, and nutrition. Each community must deal with all of these issues, and identifying approaches that are linked (such as coordinated collection of data) and are embedded within broader community planning approaches may be more effective and sustainable.

\section{Box 8.1 Integrated Cross-Sector Interventions in the Pacific Ridge to Reef Program}

The Pacific Ridge to Reef (R2R) is a Global Environment Facility (GEF) programmatic initiative involving multiple United Nations (UN) agencies to help preserve biodiversity and ecosystem services, sequester carbon, improve climate resilience, and sustain livelihoods through a ridge-to-reef management of priority water catchments in Pacific SIDS. The program recognizes the close interconnections between land, water, and coastal systems, and considers that the planning and management of freshwater use, sanitation, wastewater treatment and pollution control, sustainable land use and forestry practices, balancing coastal livelihoods and biodiversity conservation, hazard risk reduction, and climate variability and change is best achieved through integrated and coordinated efforts. It embraces the interconnections between the natural and social systems in a whole of island approach: from the ridges through coastal watersheds and habitats, and across coastal lagoons to the fringing reef environments. The R2R program stimulates effective engagement and participation of stakeholders (communities and authorities) in the planning, implementation, monitoring, and evaluation of initiatives aimed at fostering integrated approaches to natural resource and environmental management. ${ }^{a}$

a. See Pacific R2R's website, http://www.pacific-r2r.org/.

To a lesser degree, a similar approach has been taken to provide power, water, and waste services to remote and small indigenous populations in Australia. The approach appears to provide cost efficiencies and increased effectiveness relating to (i) developing and deploying human resources capacity, (ii) transporting people and resources to and from communities, (iii) economies of scale through greater purchasing power, and (iv) more efficient and integrated community engagement and planning. One critical strategy is the riskbased approach that assesses risk across multiple sectors simultaneously, which could be applied to community-scale water, waste, health, and energy systems in Pacific SIDS.

\subsubsection{Meeting Human Capacity Needs for Water Management in Remote and Small Populations}

As described in chapter 4.2.2, several recommendations have been made over the past 15 years for coordinated regional strategies to build capacity (e.g., the Pacific Regional Action Plan on Sustainable Water Management). A lesson from the health sector is that concerted and long-term investment in capacity building is required, including specific strategies to overcome isolation of small populations, and challenges of economies of scale in delivering training and education.

Some potential ways forward, specific to small remote populations, and based upon the lessons from other sectors and places, include regional strategies to explicitly build the required capacities (community, local government, national government, utilities, civil society) for water management and services of small remote populations. For instance, there can be regional platforms for free learning, delivered electronically (not necessarily online) that provide essential skills for water management and services of small, remote populations. These platforms could include country support officers who can travel to provide onsite training to rural hubs. In so doing, they could create a community of practice (connecting government, and service providers and civil society to enable peer-to-peer sharing of skills and knowledge).

Another option is to use the model of local service operators employed by centralized service providers, such as the Australian provision of services to indigenous communities. This model formalizes the skills development process for community-based staff through mentoring (remote, potentially via mobile phones) and onsite training, and formalizes the provision of technical and resources support to community water systems.

Programs could embrace the potential for emigration of newly skilled community operators by supporting skills development and continuing a pipeline of skilled community workers. For this program to work, it must 
be accepted that outmigration, temporary or permanent, is a potentially constructive mechanism for general economic development of these communities. Finally, there can be partnerships with both adult education providers and water-related academia and educators to collect data related to water skills (capacity needs assessments) to guide the development and implementation of skills development strategies to remote and small populations.

\subsection{Gaps in Knowledge}

The most significant gap is the absence of analytical knowledge specifically addressing water management and services in small remote populations. In particular, these questions may help the development and evaluation of practical strategies to address the main impacts to water management and service delivery for remote and small populations:

- What feasible, effective and sustainable mechanisms can be used to link relevant expertise to remote populations for long-term support?

- Are there any lessons from the e-health concepts in health services in which medical advice is provided to remote populations from expert professionals remotely, via local general health workers?

- What types of technical and resource capacity are required for different contexts across remote and small communities of the Pacific SIDS? How can they be developed?

- What capacity does the local government have or need to support communities? How can this be developed?

- What role could national governments or utilities play in building community and local government capacity?

- What are sustainable mechanisms to finance such service delivery models? What is the role of subsidies?

- What infrastructure and other technologies are best suited to self-supported WSS systems for small remote populations in the context of water insecurity and disaster resilience? How can effective supply chains for these systems be achieved?

- Are there benefits to integrating WSS service delivery with other sectors (e.g., energy and health)?

- What information or data should be collected to monitor and support management of small remote water systems? How can this be collected and linked back to improving services?

The importance of sociocultural geographies to overcoming the challenges that isolation and small populations present to water management is not discussed in the water-related literature, and is not apparent in the light touch review of other sectors. Given the complexity of sociocultural geography with Pacific SIDS (different islands with varied sociocultural contexts), we might expect an important influence on the appropriateness and success of any strategies to improve water management with isolated and small populations. This would be particularly relevant for regionwide or regionally centralized actions.

A political economy analysis could inform how power and resources are distributed and contested and the implications for developing water management outcomes. This type of analysis gets beneath the formal structures to reveal the underlying interests, incentives, and institutions that enable or frustrate change. Such insights are important if we are to advance challenging agendas around governance, economic growth, and service delivery, which experience has shown do not lend themselves to technical solutions alone. 


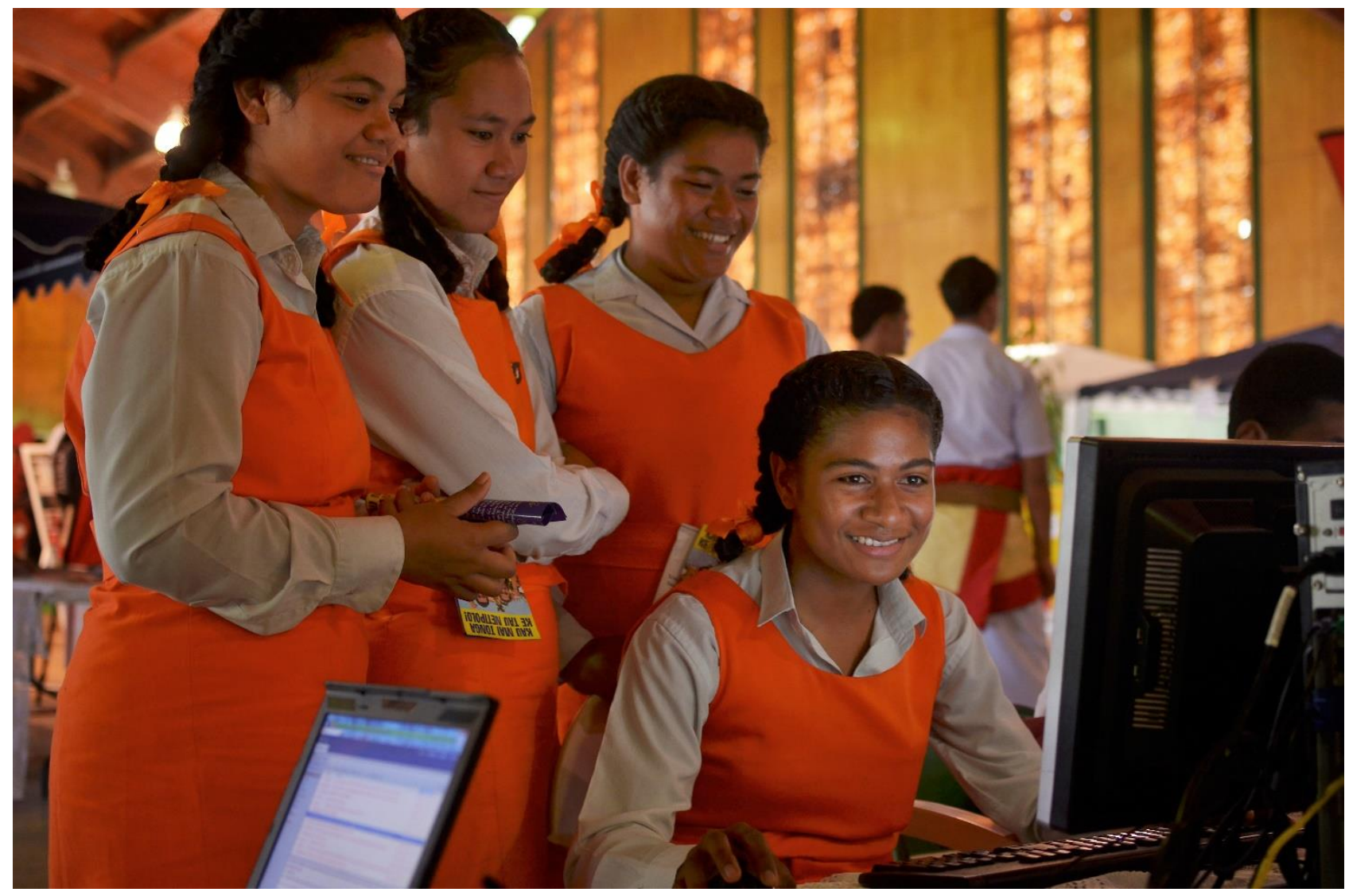

(Credit: Codrington/World Bank 2005)

\section{Water Data, Information, and Knowledge}

\subsection{Nature of the Challenge}

Available, accurate, and user-friendly information and knowledge are central to meeting water management and water supply, sanitation, and hygiene (WASH) objectives. The generation and availability of information and knowledge are also valuable in disaster risk management; drought management and water demand management; informing efforts to increase resilience and adaptive capacity, ensure adequate environmental protection, and prevent and ensure quick response to disease outbreaks; and anticipate future service delivery needs in areas experiencing rapid urbanization. Ensuring information availability implies activities such as monitoring and evaluation embedded in WASH programs to review implementation and effectiveness (Pacific WASH Coalition 2009), and environmental monitoring such as that carried out for abstraction rates and the thickness of freshwater lenses to determine groundwater availability and appropriate abstraction rates (Burke 1999).

Information and knowledge challenges in Pacific Island countries relate to the collection, sharing, analysis, and use of information and knowledge, and reliance on external support. These challenges can play out at community, national, and regional scales, and can affect various facets of WASH and water management.

\subsubsection{Collection of Information and Knowledge}

Collection of information and knowledge relating to water resources and their use is often limited in Pacific Island countries. For many water supplies, no regular water quality surveillance is conducted. Gaps in knowledge remain regarding rural and peri-urban household water management practices and how they 
may vary by season and across multiple water sources, and how this relates with use and supply of other water sources (Hadwen et al. 2016; White and Falkland 2010). In urban water supply systems, leaks can lead to substantial water losses, and there may be limited systematic monitoring and reporting to identify leaks and deteriorating water quality or to inform water demand management and drought management strategies. Water quality monitoring can be constrained by a lack of resources to carry out analysis, finances, and skilled workers (Mirti and Davies 2005).

Groundwater monitoring has been constrained by limited capacity and insufficient or nonfunctional monitoring equipment (IGRAC 2016). Islands are often separated by large distances from testing laboratories, and this can present difficulties in monitoring and assessing environmental quality at a larger geographical scale (Brodie, Lee, and Prasad 1983). In the workshop "Groundwater Monitoring in Small Island Developing States in the Pacific", participants from Pacific Island countries identified accessibility challenges of outer islands resulting in data monitoring gaps (IGRAC 2016). Scott, Ricci, and Fatai (1999) have argued that effective groundwater assessment and development has been constrained in small outer islands because of the practical difficulties of getting drilling rigs to remote places. Both the Pacific Hydrological Cycle Observing System (HYCOS) and the Transboundary Waters Assessment program (IGRAC 2016) have drawn attention to the limited baseline of groundwater assessments and the priority need for monitoring of abstraction to inform the establishing basic sustainable yields and the development of basic governance structures to support groundwater resources.

In Kiribati, a lack of baseline accessible information, irregular reporting and lack of analysis of information was considered by GEF (Global Environment Facility), the United Nations Development Programme (UNDP), and the Secretariat of the Pacific Community (SPC) to be exacerbating water management challenges. Gaps in knowledge relate to hydrology, meteorology, health, water quality and demand, environment, community attitudes, finance, and service performance (GEF 2007).

Interviews with stakeholders in Fiji and Vanuatu indicate that access to quality data poses a barrier to WASH program planning. In Vanuatu, WASH practitioners use environmental data that have not been updated for years, and they lack access to the government's water inventory. WASH practitioners in both countries express frustration about a lack of quality hazard and risk data to use in WASH program planning. They identify a need for training on data collection, storage and interpretation, and for standard assessment tools and indicators for data collection (UNICEF 2017a).

\subsubsection{Sharing of Information and Knowledge}

Limited information availability is not always a result of a lack of information. In Papua New Guinea, Nichols (2004) reports limited data availability relating to the state of the environment despite large volumes of data being collected. Here, data have not been collated into a central location, but instead are scattered and held in many Papua New Guinea-based and international institutions (Nichols 2004).

A Drinking Water Safety and Security Plan (DWSSP) program overview finds that in many Pacific Island countries there is limited monitoring of WASH project outcomes at a national level and usually no central database for WASH data (UNICEF 2016a). In Fiji, WASH practitioners saw room for improvement in sharing data relevant to their work (UNICEF 2017b). Similarly, Crennan (2001) finds that information transferred to counterparts on water management projects is not always shared with other project team members.

Water safety needs a holistic framework in which there is available knowledge and sharing of information. However, this is not always done. According to a report (Kumar 2010) information gathered from water sampling and quality tests carried out by the Fiji Water Supply Department and the Ministry of Health was not shared, resulting in duplication of tasks and lost opportunity for synergy and ensuring consistency of results. Similarly, Mirti and Davies (2005) have reported that for centralized water supply infrastructure in Pacific Island countries, feedback systems to alert water suppliers about water quality problems tend to be 
inefficient, and water quality monitoring is often ad hoc, carried out by separate agencies with limited data sharing.

\subsubsection{Analysis and Use of Information and Knowledge}

A need for strengthened capacity of governments to assess and monitor water resources has been identified by Falkland (2002a). The Pacific HYCOS project, managed by SOPAC and implemented from 2006 to 2010 in partnership with WMO and UNESCO, finds that there was limited use of monitoring data to improve efficiency of groundwater abstraction or to maintain water quality. They also find that limited reporting on water resources resulted in poor data collection and management, and presented problems for the future management and sustainability of water resources (IGRAC 2016). Participants from Pacific Island countries attending the IGRAC workshop "Advancing Groundwater Monitoring in Pacific SIDS" also report that analysis and dissemination of data could be improved (IGRAC 2016).

Governments may have limited capacity to apply climate-related information into policies and plans to manage risks associated with climate variability and change (Pacific WASH Coalition 2009). In some cases, data relating to climate change risks can be difficult to use for government personnel, hindering the development of policy and programs, and the prioritization of interventions. These difficulties have been shared with some WASH practitioners in Fiji and Vanuatu, who report they do not have the skills to integrate risk data into WASH programs (UNICEF 2017a).

The Pacific Water and Wastewater Association (see Photo 4.1) supports data collection from utilities of the region, identified operational trends, helps organizations benchmark data, analyze strengths and weaknesses and train them to improve their performance.

\section{Photo 4.1: Annual conference of the Pacific Water and Wastewater Association in Tonga (2016)}

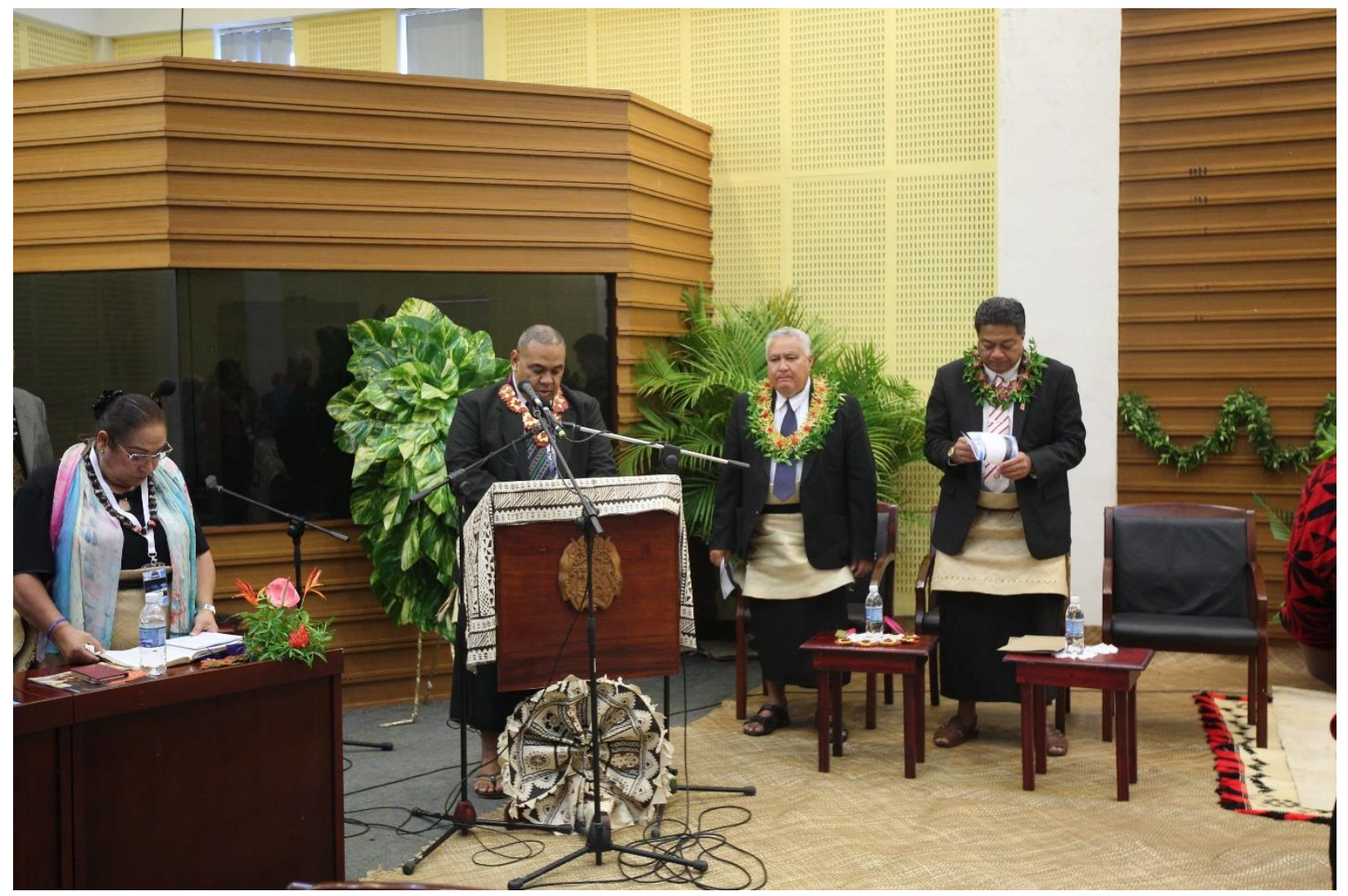

Credit: Alexander Danilenko/World Bank 


\subsubsection{Reliance on External Knowledge and Information Analysis}

In Vanuatu, Poustie and Deletic (2014) find that limited knowledge of national government staff, especially in the field of engineering, has led to reliance on external sources of knowledge when technical decisions are needed. They argue that limited knowledge and experience in design, construction, and implementation of structural measures has constrained infrastructural improvements in Port Vila.

\subsection{Ways Forward}

\subsubsection{Island-and Community-Level Ways Forward}

Literature points to the importance of using local knowledge in water resource assessments (Falkland 2002c) and in the development of plans and projects. This contributes to their quality and gives the community a sense of ownership (Burke 1999). In addition, community participation in water management has the potential to substantially improve understanding and awareness of the relationship between water and health. Capacity building for community workers and communities in the use of participatory approaches (Mirti and Davies 2005) and in carrying out water resource assessments (Falkland 2002c) can foster enhanced awareness and information sharing (Mirti and Davies 2005).

However, in some rural and remote communities, knowledge relating to health risks of unsafe drinking water can be poor, especially in relation to children's health (Mirti and Davies 2005). In such cases it is proposed that role models and community leaders can be mobilized to share knowledge regarding WASH, sustainable management of water, or climate resilient water systems (UNICEF 2017a). Crennan (2001) recommends dedicating time with counterparts one-to-one to make sure there is shared understanding and knowledge transfer.

Hydrogen sulfide testing in Palau and outer islands of the Marshall Islands has been carried out to enable water quality monitoring of private household rainwater tanks and communal water supplies. Because the water quality test is cheap and reliable, it is a feasible method to ensure water quality (Mirti and Davies 2005).

Dworsky (2002) points out that imparting knowledge is not just about giving information. Consultants with technical skills are often tasked with transferring knowledge and capacity to stakeholders. Yet capacity building is a skill that consultants with technical expertise might not necessarily have. Aitaro (2007) and Crennan (2001) have suggested that water practitioners and consultants avoid putting information in lengthy reports when working with communities that do not have a culture of using written materials at length. They also recommend avoiding writing reports or materials that do not acknowledge existing capacity and knowledge in Pacific Island countries at national and community levels. Crennan (2001) suggests using active demonstrations and workplace presentations, or creative methods such as images, theater, or video.

The Solomon Islands Water Sector Adaptation Program (SIWSAP) uses community knowledge to inform climate change adaptation and risk management plans. Location-specific reports have been prepared on climate change predictions. These can be used by community members to produce risk reduction management plans that integrate local knowledge and internationally verified climate science (UNICEF 2017a).

Another good example of integration of locally and externally derived information is a sanitation project carried out by Tuvalu International Waters Project (IWP). The project engaged a community in information collection and knowledge building across external researchers and households. Following problem identification between researchers and community members, baseline and water quality assessments were carried out to confirm problem identification. Researchers collated information on community attitudes, methods of awareness raising, merits and disadvantages of alternative sanitation technologies, and 
waterborne diseases in the community. This was done with a high level of community participation, and was followed by training of a cross-section of the community on the health, economic, and technical issues of wastewater management. The project is said to have increased awareness of wastewater management problems and the development of appropriate solutions (Crennan 2007a).

Routine monitoring of drought conditions in Rarotonga has been carried out by the Water Works Department to inform drought management strategies. This monitoring is intended to provide insights into drought severity and assist public awareness of water supply conditions and requirements for conservation (Burke 1999). The South Tarawa Drought Response Plan uses a drought index based on rainfall monitoring to trigger drought alerts and appropriate timely actions for water conservation (Falkland 2011; White et al. 1999).

Device-based surveys have been used to better understand patterns of water use across different seasons and during flood and drought events in flood prone catchments in the Solomon Islands, and drought prone atolls in the Marshall Islands (Hadwen et al. 2016). The device-based survey assists the rapid collection and immediate access to complex multiple water source data from communities, with the potential to assist policy makers to identify risks for disaster risk management and climate change adaptation in real time (Hadwen et al. 2016).

\subsubsection{Country-Level Identified Ways Forward}

There have been substantial efforts to increase the capacity of Pacific Island countries to monitor and assess the status and trends of their water resources; this can be used to inform hazard warnings, infrastructure development, and environmental protection (IGRAC 2016). This includes making sure information gleaned postdisasters (such as extreme weather events and disease outbreaks) is used to enhance disaster risk reduction activities. Free access to information and regular analysis and reporting of information relating to numerous aspects of water systems are needed (GEF 2007).

Mirti and Davies (2005) have recommended the establishment of legislation or national water quality standards that require water quality checks or surveillance. Some countries and territories have established legislation and standards relating to water quality monitoring already (including Papua New Guinea, Palau, and American Samoa). In Fiji, the groundwater monitoring reports are developed every five years in accordance with water policies and government legal mandates. The reports consolidate all groundwater data and integrate groundwater and surface water data into a basin catchment analysis. Groundwater data are collated in a central database (IGRAC 2016).

Graphic information systems (GIS) software is later used in data analysis and integration of government groundwater monitoring in Fiji (IGRAC 2016). The recording of water system data in a GIS platform that is periodically updated and that can be used by various government departments and civil society groups has been proposed by Burke (1999) as a useful way to integrate and share data across authorities and government departments. Remote sensing can be used to map changes in environment, land use changes, town planning, surface water resources, vegetation, water quality, coastal area, mangrove coverage, and evapotranspiration. This is becoming more widely used in Carribean SIDS: Barbados, Haiti, and the Dominican Republic. It helped, for instance, assess the reason for lake flooding in Haiti, and was used to produce a storm

surge hazard map in Jamaica. It is important to note that the capacity to analyze remote sensing data was built in-country in those cases. (UNESCO 2014)

National forums can enable sharing of data and information as well as successful models and technologies relevant to WASH and water management. In Fiji and Vanuatu, efforts are being made by national clusters to improve information gathering and sharing. UNICEF has sought to support data collection and analysis through the online tool Akvo (UNICEF 2017b).

The potential benefits of tools and information to improve the efficiency and monitoring of WASH programs and put in place DWSSPs have been raised by the UNICEF Pacific WASH team. This includes methodologies 
that can be used by multiple partners and allow for the monitoring of projects (UNICEF 2016a). Tools and methodologies such as these may help to support evidence-based systems that can enable prioritized investments in WASH and inform strategic decisions relating to policy, budget needs, and institutional arrangements (UNICEF 2017b).

\subsubsection{Regional Ways Forward}

Regional frameworks are needed for knowledge and information sharing, and the exchange of lessons learned and best practice relating to resilience and other cross-cutting issues affecting water security in the Pacific (UNICEF 2017b). The Pacific WASH Coalition was established informally in 2007 to collaboratively support regional initiatives relating to WASH. Its membership includes the Foundation of the Peoples of the South Pacific International (FSPI), the Fiji School of Medicine (FSMed), Live \& Learn Environmental Education (LLEE), the World Health Organization (WHO), the International Federation of Red Cross and Red Crescent Societies (IFRC), UN-Habitat, UNICEF, and the Pacific Islands Applied GeoScience Commission (part of SOPAC). Members benefit from sharing of knowledge, information, and experiences, sharing access to technical resources and materials, and contributing to lessons learned and drawing on best practice. The coalition has facilitated feedback and in-country visits through information and field trip reports, and has promoted incountry coalitions. The Pacific WASH Coalition (2009) prepared a drinking water quality and health framework for Pacific Ministries of Health to consider implementing, including the following recommendations:

- Provide technical support to develop national drinking water quality standards.

- Enhance human resources for drinking water quality monitoring, data management, and information systems.

- Conduct further research for evidence-backed drinking water quality and health plans and policies.

- Promote community awareness raising efforts relating to water quality and health risks, source water protection, and water treatment.

- Conduct community-based water quality testing.

- Provide technical assistance and training to strengthen water quality monitoring.

- Ensure sufficient and reliable equipment for water quality monitoring and hardware and software for data management, including appropriate field test kits for remote and rural areas.

- Strengthen communication and information exchange between agencies involved in water quality data collection, including exchange and joint analysis of drinking water quality data and disease surveillance data between water supply and health authorities.

At a recent workshop held by IGRAC, attended by groundwater managers, participants discussed the potential benefits of improving the Tideda database for groundwater in small island developing states (SIDS). The Tideda database is useful but has not enabled successful management of groundwater data. Possibilities of either developing a new database or improving the Tideda database were discussed (IGRAC 2016). In Vanuatu, local stakeholders have expressed interest in learning from other countries and developing water resources in a manner that "leapfrogs" water system deficiencies in upper-middle-income countries (UMICs) (Poustie and Deletic 2014).

\section{Box 9.1 Non-Pacific SIDS-Global Platforms for WASH and Water Resource Management Data in SIDS}

The Water Point Exchange data have some data on water point monitoring (boreholes, wells) for SIDS (Haiti, East Timor). ${ }^{a}$ This is similar to the tool Akvo, but open source. The Joint Monitoring Programme (JMP) has some data for SIDS on access to WASH services. ${ }^{b}$ Sistema de Información de Agua y Saneamiento Rural (SIASAR) is a World Bank- 
funded platform used for rural water supply and sanitation (WSS) data and monitoring in Latin American countries, including some SIDS (the Dominican Republic). ${ }^{c}$

The World Bank's DRM platform ThinkHazard provides a general view of the hazards for a number of SIDS that should be considered in project design and implementation to promote disaster and climate resilience. The tool highlights the likelihood of different natural hazards affecting project areas (very low, low, medium, and high), provides guidance on how to reduce the impact of these hazards, and where to find more information. The hazard levels are based on published hazard data, provided by a range of private, academic, and public organizations. ${ }^{d}$ Water-related hazards include river floods, urban floods, coastal floods, and water scarcity. Information on hydrogeology of African SIDS (e.g., the Comoros) has been collated in the Africa Groundwater Atlas, available online and offline to be able to use it in a context with low Internet bandwidth. ${ }^{\mathrm{e}}$ The accompanying Africa groundwater literature archive has collated information and research papers for groundwater in African SIDS (Comoros) and made it freely available to all. ${ }^{\dagger}$

a. See Water Point Exchange's website, https://www.waterpointdata.org/.

b. See WASH data on the JMP website https://washdata.org/.

c. See the SIASAR website, http://www.siasar.org/en.

d. See for example the World Bank ThinkHazard website, http://thinkhazard.org/en/report/262-vanuatu/DG.

e. See the Africa Groundwater Atlas website, https://www.bgs.ac.uk/africagroundwateratlas/.

f. See the Africa Groundwater Atlas website index, https://www.bgs.ac.uk/africagroundwateratlas/index.cfm.

\subsection{Gaps in Knowledge}

Gaps in knowledge include the following:

- Frameworks to guide the collation, dissemination, and analysis of data relevant to climate resilient WASH (Hadwen et al. 2016).

- Groundwater assessments for different contexts: outer islands, atolls, and rapidly urbanizing areas.

- Frameworks for linking data and information relating to groundwater and surface water.

- Identification of monitoring techniques, equipment, data collation, data sharing and analyses appropriate for outer islands and remote areas.

- Identification of methods of integrating community-level information and knowledge and monitoring data with national-level water resource assessments and policy making.

- Applicable citizen-science models that can be coordinated with water resource assessments and monitoring in Pacific SIDS.

- Identification of communication methods to capture and share water information that are culturally and socially appropriate (few literature refers to systematic collection or application of traditional and locally held knowledge relating to water systems and water management).

- Methods and training for external consultants offering technical support to increase their capacity to share information and knowledge in constructive ways. 


\section{Appendix A. Methodology}

\section{A.1 Sourcing Knowledge}

This review was a desk-based exercise, limited to documented analytical knowledge, in particular published and easily accessible documents. No site visits of stakeholder consultations were conducted for this review. Documents were gathered from a variety of sources, including the following:

- Web of Science database of peer-reviewed publications

- Online libraries of the Secretariat of the Pacific Community (SPC) and the Secretariat of the Pacific Regional Environment Program (SPREP)

- University of South Pacific, including two associated journals: South Pacific Journal of Natural Sciences and Journal of Pacific Studies

- Targeted Internet searches for known authors or groups

- Targeted Internet searches on specific topics for which resources weren't identified using the preceding strategies

- Authors and key contacts at the World Bank and UNICEF (United Nations Children's Fund)

The search terms used with these sources include the list of Pacific small island developing states (SIDS) (see following paragraph) with freshwater or fresh water or water resources, sanitation, irrigation, water and economy, water or community and natural resources management, water and community management, water and health, water and governance, economies of scale, water and remote, water and services.

In additional to searching for Pacific Islands in combination with the above search terms, the following Pacific SIDS were specifically included in the search strategy: Fiji, Kiribati, the Marshall Islands, the Federated States of Micronesia, Nauru, Niue, Palau, Papua New Guinea, Samoa, the Solomon Islands, Tonga, Tuvalu, Vanuatu.

The types of documents include peer-reviewed publications (in journals and conferences), analytical reports and briefs (commissioned or research), workshop reports, program reports or communiques, book sections, discussion papers, handbooks and guidelines, and theses. The types of knowledge include primary research, evaluations, reviews, program descriptions, guidance for policy makers, programs or practitioners, workshop and consultation syntheses, and opinion pieces.

\section{A.2 Characterizing and Prioritizing Documents}

All documents obtained were initially screened. All but a very few irrelevant documents are included in the document library and annotated bibliography. The following information about every document is recorded in the annotated bibliography:

- CODE (for easy location within the library))

- Title

- Authors

- Editors

- Year

- Source

- ISBN

- Document type

- Knowledge type

- Objective (stated objective of work doc) 
- Geographical focus

- Keywords

- Abstract (if greater than 300 words)

- Key conclusions

- Relevance of document (based reviewing the abstract and applying the following criteria: A: highly relevant and key document addressing multiple water issues and locations; B: highly relevant but narrower focus, addressing fewer water issues or locations; C: relevant, but narrower focus and scale; D: marginal value to this review)

- Water-related challenges (environmental variability and change; isolation and remoteness; small scales; social and cultural; integration and coordination, human capacity, urbanization, information, and knowledge; service delivery)

- Type of water resource*6 (groundwater, rainwater, surface water, desalinated water, wastewater, piped water, bottled water)

- Water use* (domestic or municipal, household, industry or commercial, irrigation, mining, fishing, drainage)

- AWDO Water Security dimension* (household, urban, economic, environmental and disaster resilience)

- Sustainable Development Goals (SDGs)* (if relevant to SDG6).

- IWRM component* (water resource assessment; policy and strategy; implementation plans and programs; monitoring and evaluation; governance arrangements and community participation)

The process of reviewing abstracts further refines the list of water-related challenges, which forms the structure for summarizing the analytical knowledge.

\section{A.3 Summarizing Analytical Knowledge}

For each water-related challenge topic, the highly relevant documents were rapidly reviewed and synthesized to generate a summary of the common and critical aspects relating to (i) the nature of the challenge (description of the problems and causes); (ii) identified ways forward (summary of key recommendations for addressing this challenge); and (iii) gaps in analytical knowledge (summary of critical gaps already identified by document authors, or critical gaps identified by the authors of this review). A brief overview of the nature of the documents included in the rapid review for each challenge topics is also included.

\section{A.4 Inputs from Non-Pacific SIDS}

A rapid desk review was undertaken in parallel, focusing on water management in non-Pacific SIDS: those located in the Caribbean, Atlantic and Indian Oceans. The first step was to compile an inventory of relevant analytical work focused on aspects that relate directly to SIDS context (e.g., scale, isolation, hydrological and coastal issues, climate resilience, or low capacity), published either in academic journals or in the grey literature. These were identified through Internet searches for known organizations working or particular keywords, combined with the targeted SIDS. The knowledge collected was compiled using the same methodology as for the Pacific SIDS review and includes for each reference: (i) the country(ies) it relates to; (ii) the subsector(s); (iii) title and reference; (iv) short summary (about 100-200 words) of key topics and

${ }^{6}$ The asterixed characteristics were added to improve the usefulness of the annotated bibliography for purposes beyond this review. Due to time constraints, some of these characteristics are incomplete for some of the lower-relevance documents. 
findings; and (v) key words. A total of 43 documents were analyzed. These provide the basis for the boxed examples of non-Pacific SIDS elements, which complement and put in perspective findings related to Pacific SIDS.

\section{A.5 Availability of Analytical Knowledge}

A total of 498 documents and resources are in the annotated bibliography. The accompanying electronic library contains a copy of most of these (both the annotated bibliography and library have been provided in electronic form to the World Bank). A major review of knowledge, both explicit and tacit, relating to sustainable water management across Pacific Island countries was undertaken 2002, and is a significant and relevant resource Tables A.1 and A.2 describe the breakdown of documents by geographic area they cover, thematic focus and level of relevance for this review.

Table A.1 Number of documents by geographic area by level of relevance (A being the highest)

\begin{tabular}{|l|l|l|l|l|l|}
\hline Country & A & B & C & D & Total \\
\hline Fiji & 14 & 14 & 25 & 20 & 73 \\
\hline Kiribati & 15 & 16 & 19 & 8 & 58 \\
\hline Marshall Islands & 10 & 9 & 3 & 11 & 33 \\
\hline Micronesia, Fed. Sts. & 9 & 11 & 11 & 7 & 38 \\
\hline Nauru & 10 & 5 & 2 & 3 & 20 \\
\hline Niue & 10 & 5 & 4 & 3 & 22 \\
\hline Palau & 8 & 5 & 2 & 5 & 20 \\
\hline Papua New Guinea & 12 & 9 & 29 & 24 & 74 \\
\hline Samoa & 10 & 7 & 6 & 10 & 33 \\
\hline Solomon Islands & 14 & 8 & 9 & 13 & 44 \\
\hline Tonga & 13 & 8 & 7 & 11 & 39 \\
\hline Tuvalu & 13 & 6 & 7 & 3 & 29 \\
\hline Vanuatu & 16 & 8 & 5 & 8 & 37 \\
\hline Pacific Island countries & 31 & 144 & 17 & 14 & 206 \\
\hline
\end{tabular}

Table A.2 Number of documents by thematic area and by level of relevance (A being the highest)

\begin{tabular}{|l|l|l|l|l|l|}
\hline Challenge & A & B & C & D & Total \\
\hline $\begin{array}{l}\text { Remote and small populations and } \\
\text { economies of scale }\end{array}$ & 4 & 6 & 3 & 2 & 15 \\
\hline $\begin{array}{l}\text { Environmental variability and climate } \\
\text { change }\end{array}$ & 22 & 20 & 46 & 38 & 126 \\
\hline Urbanization and water & 18 & 19 & 40 & 19 & 96 \\
\hline Socioculture and water & 27 & 39 & 62 & 24 & 152 \\
\hline WASH service delivery & 20 & 10 & 39 & 22 & 91 \\
\hline Water data, information and knowledge & 18 & 17 & 33 & 21 & 89 \\
\hline $\begin{array}{l}\text { Water sector governance, coordination, } \\
\text { integration and capacity }\end{array}$ & 17 & 15 & 24 & 10 & 66 \\
\hline
\end{tabular}

Note: $\mathrm{WASH}=$ water supply, sanitation, and hygiene.

The following subsections provide overviews of analytical knowledge used in the various sections of this report.

\section{A.5.1 Environmental Variability and Climate Change}


The review identifies 118 documents relevant to environmental variability and climate change. Almost include issues relating to pollution. Fifty-three academic papers and journal articles and 50 reports relate to environmental variability and climate change. The remaining documents consist of several conference papers or are either a project appraisal or brief, brochure, funding proposal, manual, unpublished manuscript, or policy description or review.

Most documents (57) include analysis based on primary research and evaluation. Of these, over half include some reference to pollution, with roughly a quarter relevant to climate change and to freshwater lenses and groundwater management. Just under a third of the documents relating to environmental variability and climate change provide a review, while 10 provide program descriptions; and a handful are based on workshop syntheses or guidelines for practitioners.

More than half of the documents relevant to environmental variability and climate change were published within the last 10 years (66 documents). Of these relevant documents (classified as A or B, see appendix A, table A.1), most (44) were published over 10 years ago. Seventy-five of the documents focus on individual countries, of which 37 are Polynesian. Eighteen focus on multiple countries, 23 focus on the Pacific region, and the remaining refer to the Asia Pacific or a global scale.

\section{A.5.2 Water Sector Governance, Coordination, Integration and Capacity}

The review identified approximately 25 publications that are highly relevant to water sector governance, coordination, integration, and capacity. Around two-thirds of these are reports, around one-third are spread across peer review types of publications such as journal articles and conference papers, and there are a small number of guidance documents (briefing notes and guidance manuals). Significantly, much of the analysis relating to sectoral capacity, coordination, and integration are linked with the development of plans to address these issues. Several plans, particularly for regional initiatives, exist but appear not to have been wholly implemented.

Almost all publications address this challenge by reviewing situations in multiple, or all, Pacific Island countries. The few publications that are country-specific typically include insights applicable to the region. Most of the key publications were produced from 2000-05. Worth noting is the major syntheses of knowledge and stakeholder opinions relating to water management in the Pacific (Carpenter and Lawedrau 2002; Scott et al. 2003), and the 2004 review of integrated water resource management (IWRM) in Pacific Island countries (Carpenter and Jones 2004). These include several resources that describe issues and ways forward relating to integrated and coordinated water management and human capacity. Few new analyzes have been produced in the past 10 years, but many of the findings and recommendations of 2002-03 are likely still relevant.

\section{A.5.3 WASH Service Delivery}

Apart from national statistics describing access to water supply and sanitation (WSS), the rapid review has identified only one analysis throughout the region-in Papua New Guinea-of service delivery across populations, that is, urban (domestic, institutional, public and commercial, informal urban and peri-urban) and rural and remote populations. Much of the following review is therefore based upon resources dealing with these market segments separately. In addition, very few resources deal directly with analyzing existing or potential service delivery models. The concept of service delivery, rather than a focus on infrastructure provision, is not yet evident in most of the Pacific Island country resources. There is a reasonable amount of analytical information relating to urban water and wastewater utilities, though only a few relating to informal settlements that might be served by utilities. Much of the information relating to rural water services is focused upon management of groundwater in atolls (both urban and rural).

Dworsky (2002) has prepared a significant synthesis of knowledge and stakeholder opinions relating to water and wastewater utilities and their service performance in the Pacific. Approximately 20 other documents 
contain references to water service delivery in Pacific Island countries, though very few are focused on this topic.

The Pacific Water and Wastewater Association coordinates and supports annual benchmarking of utility service delivery to drive improved performance by utilities and provide performance data to national and regional stakeholders and to development partners. These benchmarking reports provide a detailed summary of different aspects of service delivery performance and institutional arrangements.

\section{A.5.4 Sociocultural Aspects of Water}

This review identified more than 150 resources, including knowledge relating to sociocultural aspects of water. Around half of these are from the last 10 years. Some are focused on a single country, and many are multicountry: either subregional or Pacific countries as a group. Around 50 are highly relevant and based upon research or evaluation, rather than program descriptions or opinions, and of those 50 around 20 appear to be peer-reviewed through formal processes.

\section{A.5.5 Unplanned Urbanization and Water}

Ninety-one documents have been reviewed relating to urbanization. They include 35 academic papers (journal articles and conference papers) and 47 reports (of which many were published with assistance from the Secretariat of the Pacific Regional Environment Programme (SPREP). The remainder are mostly plans and policy documents. Analyses in these documents are mostly based on review of information and situations (40), primary research and evaluation (36), or program description (10). Most of the remaining documents are based on guidance materials and information for practitioners.

Around a fifth of the documents relate to urbanization in the Pacific region. Several relate to urban issues at the Asia Pacific or global scale. Under a fifth discuss multiple Pacific Island countries (often focusing on Melanesian countries), while over half focused on individual countries (Fiji and Kiribati being the most common). Roughly half of the documents reviewed relating to urbanization issue were published from 2010 onward.

\section{A.5.6 Small and Remote Populations and Water}

The rapid review did not identify resources specifically dedicated to analyzing the relationships between isolation, limited economies of scale, water management and water-related industries specifically. Some opinions and observations are made within resources addressing broader water issues within Pacific small island developing states (SIDS).

The ways forward discussed herein are extrapolated and interpreted from general observations about nonwater services to remote and small populations within Pacific SIDS. In addition, a very light touch review of resources relating to sustainable water services for small remote populations identifies some relevant recommendations.

\section{A.5.7 Water Data, Information and Knowledge}

The review has identified 89 documents relevant to the topic of information and knowledge. Just under half of the documents reviewed are reports. Most of the remaining documents are academic papers. Documents reviewed also include a small number of guidance notes and manuals for practitioners, book sections, appraisals, synopses, and even a comic. Roughly half of the reviewed documents were published within the past 10 years. Just under half of the documents are based on primary research or evaluation, and under a third refer to reviews of information and situations. Reviewed documents also include a handful of program descriptions and one workshop synthesis. 
Most documents relate to specific countries, with Papua New Guinea as the most common. Over 20 documents focus on the Pacific region, with several viewing issues at an Asian Pacific or global scale. The remaining documents refer to multiple countries. 


\section{Bibliography}

Aarons, A. 1983. "Teachers and Health Workers: Partners in Primary Health Care. Experiences from Papua New Guinea." Community Development Journal 18 (2): 132-8.

ADB (Asian Development Bank). 2008. Skilling the Pacific. Mandaluyong, Philippines: ADB.

-- - 2012a. The State of Pacific Towns and Cities: Urbanization in ADB'S Pacific Developing Member Countries. Mandaluyong, Philippines: ADB.

-- . 2012b. Summary Subsector Assessment: Higher Education, Higher Education in the Pacific Investment Program: Report and Recommendation of the President. Mandaluyong, Philippines: ADB.

---. 2014. Water Operators Partnerships Case Study: Twinning Works for Water PNG. Mandaluyong, Philippines: ADB.

---. 2016. Asian Water Development Outlook 2016: Strengthening Water Security in Asia and the Pacific. Mandaluyong, Philippines: ADB.

Aitaro, Joseph, Lowell Alik, Ritia Bakineti, Sione Fakaosi, Sione Leolahi, Narua Lovai, Patrick Mesia, Leah Nimoho, Maturo Paniani, Tauraki Raea, Kelesoma Saloa, Sandeep K. Singh, and Andy Tafileichig. 2007. Lessons for Pacific Islands Environmental Initiatives: Experiences from IWP National Coordinators. Apia, Samoa: SPREP.

Allibone, R., S. J. Cronin, D. T. Charley, V. E. Neall, R. B. Stewart, and C. Oppenheimer. 2012. "Dental Fluorosis Linked to Degassing of Ambrym Volcano, Vanuatu: A Novel Exposure Pathway." Environmental Geochemistry and Health 34 (2): 155-70.

Alto, W. A., and L. B. Nettleton. 1989. "Hydatid Disease: The Threat within Papua New Guinea." Papua New Guinea Medical Journal 32 (2): 139-42.

Aqorau, Transform. 2016. "State of the Pacific-Slippery Slopes and Rough Rides in Regional Cooperative Endeavours in the Islands." SSGM Discussion Paper, Australian National University, Canberra, Australia.

Asari, Y., Y. Koido, K. Nakamura, Y. Yamamoto, and M. Ohta. 2000. “Analysis of Medical Needs on Day 7 After the Tsunami Disaster in Papua New Guinea." Prehospital and Disaster Medicine 15 (2): 9-13.

Aung, Than, Awnesh Singh, and Dayang Siti Maryam. 2011. "Sea Level Changes in the Pacific Region and Impacts of the 2009 El Niño in Fiji Waters [Assessment from 18 Years Land-Based Data]." South Pacific Journal of Natural and Applied Sciences 29: 26-30.

AusAID. 2012. "Social Cohesion in the Pacific." AusAID, Canberra, Australia.

Bailey, R. T., and J. W. Jenson. 2013. "Effects of Marine Overwash for Atoll Aquifers: Environmental and Human Factors." Ground Water 52 (5): 694-704.

Bailey, R. T., J. W. Jenson, and D. Taboroši. 2013. "Estimating the Freshwater-Lens Thickness of Atoll Islands in the Federated States of Micronesia." Hydrogeology Journal 21 (2): 441-57.

Baines, G. B. K. 2000. Traditional Resource Management in the Melanesian South Pacific: A Development Dilemma. Apia, Samoa: SPREP.

Bargh, Brian J., and Jac J. Baru. 1982. "Water Quality and Village Water Supplies of the Ramu River Above Yonki, Eastern Highlands (Papua New Guinea)." Science in New Guinea 9 (1): 27-37.

Barrington, D. J. 2016. "Inadequate WaSH in the South Pacific: How Might It Be Impacting Children?" Reviews on Environmental Health 31 (1): 159-62. doi:10.1515/reveh-2015-0034. 
Barrington, D. J., J. Bartram, S. Meo, S. G. Saunders, K. F. Shields, S. Sridharan, and R. T. Souter. 2017. Fostering Water, Sanitation and Hygiene (WaSH) Marketing Exchanges Using Participatory Processes: A Guide for Working with Residents of Informal Settlements in the Pacific. Brisbane, Australia: International Water Centre.

Barrington, D. J., S. Sridharan, S. G. Saunders, K. F. Shields, S. Meo, R. T. Souter, and J. Bartram. 2017. "Programming Brief: Using Participatory Processes to Engage Informal Settlements and Enabling Actors in Improving WaSH." International Water Centre, Brisbane, Australia.

Barrington, D. J., S. Sridharan, S. G. Saunders, R. T. Souter, J. Bartram, K. F. Shields, S. Meo, A. Kearton, and R. K. Hughes. 2016. "Improving Community Health through Marketing Exchanges: Insights from a Participatory Action Research Study on Water, Sanitation, and Hygiene in Three Melanesian Countries." Social Science \& Medicine 171: 84-93. doi:10.1016/j.socscimed.2016.11.003.

Barrington, D. J., S. Sridharan, R. T. Souter, S. G. Saunders, K. F. Shields, S. Meo, and J. Bartram. 2017. "Programming Brief: Building on Existing Marketing Exchanges in Melanesian Informal Settlements to Improve WaSH." International Water Centre, Brisbane, Australia.

Beatty, Mark E., Tom Jack, Sumathi Sivapalasingam, Sandra S. Yao, Irene Paul, Bill Bibb, Kathy D. Greene, Kristy Kubota, Eric D. Mintz, and John T. Brooks. 2004. “An Outbreak of Vibrio Cholerae O1 Infections on Ebeye Island, Republic of the Marshall Islands, Associated with Use of an Adequately Chlorinated Water Source." Clinical Infectious Diseases 38 (1): 1-9.

Becker, Chris. 2012. "Small Island States in the Pacific: The Tyranny of Distance?" IMF Working Paper 12/223, IMF, Washington, DC.

Belmar, Yasa N., Karen E. McNamara, and Tiffany H. Morrison. 2016. "Water Security in Small Island Developing States: The Limited Utility of Evolving Governance Paradigms." WIREs Water 3: 181-93.

Benezit, M. 1998. Report on the Mining Pollution in New Caledonia. Apia, Samoa: SPREP.

Berg, S. 2014. "Performance Improvements for Caribbean WSS Utility Companies." Presented at the Water Utility reform Workshop, Bridgetown, Barbados, December.

Berlioz-Arthaud, Alain, Tom Kiedrzynski, Narendra Singh, Jean-Francois Yvond, Gwenael Roualen, Cyril Coudert, and Vasiti Uluiviti. 2007. "Multicentre Survey of Incidence and Public Health Impact of Leptospirosis in the Western Pacific." Transactions of the Royal Society of Tropical Medicine and Hygiene 101 (7): 714-21.

Bower, Rhonda, and Clive Carpenter. 2003. "Key Issues in Water Security and Sustainable Development in the Pacific." Development Bulletin 64, Australian National University, Canberra, Australia.

Bower, Rhonda, Leonie Crennan, and Arieta Navatoga. 2005. The Sanitation Park Project. Suva, Fiji: SOPAC.

Branchoux, Candice, Lin Fang, and Yusuke Tateno. 2017. Estimating Infrastructure Financing Needs in AsiaPacific Least Developed Countries, Landlocked Developing Countries and Small Island Developing States. Bangkok, Thailand: UNESCAP.

Brewster, D. R. 2006. "Critical Appraisal of the Management of Severe Malnutrition: 3. Complications." Journal of Paediatrics and Child Health 42 (10): 583-93.

Brodie, J. E., G. W. Lee, and R. A. Prasad. 1983. "Well Water Quality in South Pacific Island States." South Pacific Journal of Natural Science 4: 14-32.

Bronders, J., and J. Lewis. 1994. "Water Resources Problems on Small Islands in Fiji." Presented at the Water Down Under Conference, Adelaide, Australia, November.

Brown, J. A. H. 1987. "Estimating Streamflows for Water Development Projects in Tropical Regions." National Conference Publication, Engineers Australia, Barton, Australia. 
Brown, Rebekah, Briony Rogers, and Lara Werbeloff. 2016. Moving Toward Water Sensitive Cities: A Guidance Manual for Strategists and Policy Makers. Clayton, Australia: CRC for Water Sensitive Cities.

Bryant-Tokalau, Jennifer Joy. 2014. "Urban Squatters and the Poor in Fiji: Issues of Land and Investment in Coastal Areas." Asia Pacific Viewpoint 55 (1): 54-66.

Bukenya, G. B., T. Barnes, and N. Nwokolo. 1991. "Low Birthweight and Acute Childhood Diarrhea: Evidence of Their Association in an Urban Settlement of Papua New Guinea." Annals of Tropical Paediatrics 11 (4): 357-62.

Bukenya, G. B., and N. Nwokolo. 1990. "Transient Risk Factors for Acute Childhood Diarrhea in an Urban Community of Papua New Guinea." Transactions of the Royal Society of Tropical Medicine and Hygiene 84 (6): 857-60.

- - . 1991. "Compound Hygiene, Presence of Standpipe and the Risk of Childhood Diarrhea in an Urban Settlement of Papua New Guinea." International Journal of Epidemiology 20 (2): 534-9.

Bukenya, G. B., R. Kaser, and N. Nwokolo. 1990. “The Relationship of Mothers' Perception of Babies' Faeces and Other Factors to Childhood Diarrhea in an Urban Settlement of Papua New Guinea." Annals of Tropical Paediatrics 10 (2): 185-9.

Buluswar, Shashi, Zach Friedman, Priya Mehta, Subarna Mitra, and Roger Sathre. 2014. "50 Breakthroughs: Critical Scientific and Technological Advances Needed for Sustainable Global Development." LIGTT (Institute for Globally Transformative Technologies), Berkeley, California.

Burke, Ed. 1998a. "Demand Management and Conservation Investigations on the Island of 'Eua, Kingdom of Tonga." SOPAC, Suva, Fiji.

- - . 1998b. "Demand Management and Conservation Investigations in Funafuti, Tuvalu Demand Management Project." SOPAC, Suva, Fiji.

- - . 1999. "Recommendations for Disaster Preparedness of Water and Sanitation Systems in Pacific Small Island Developing States." SOPAC, Suva, Fiji.

Burke, Ed, and Harald Schölzel. 1998. "Demand Management and Conservation Project Field Investigations, Solomon Islands Water Authority." SOPAC, Suva, Fiji.

Butler, David J. 2004. National Assessment of the Priority Environmental Concerns of Niue. Apia, Samoa: SPREP.

Cangiano, Alessio, and Andreea R. Torre. 2016. "Migration, Gender and Politics of Development in Pacific Islands: An Introduction to the Special Issue." Journal of Pacific Studies 36 (1): 5-20.

Caplen, P. H. J. 1990. "Micro Hydro for an Isolated Rural Water Supply." International Water Power and Dam Construction 165: 34.

Carden, Yale R. 2013. "Solid Waste-Level Rise on Atoll Nation States: A Less Publicised Environmental Issue in the Republic of Kiribati." Australasian Journal of Environmental Management 10 (1): 35-45.

Carpenter, Clive, and Paul Jones. 2004. An Overview of Integrated Water Resources Management in Pacific Island Countries: A National and Regional Assessment Status Report for GWP. Suva, Fiji: SOPAC.

Carpenter, C., and A. Lawedrau. 2002. "Effects of Forestry Activities on Surface Water Quality in the Pacific Region: A Case Study of the Rewa River Catchment, Fiji Islands." International Forestry Review 4 (4): 307-9.

Carrard, N., J. Crawford, G. Halcrow, C. Rowland, and J. Willetts. 2013. "A Framework for Exploring Gender Equality Outcomes from WaSH Programmes." Waterlines 32 (4): 315-33. 
Carroll, D. G., P. Ula, A. J. Marr, B. Kipit, and N. Davis. 1994. "Aspects of Stormwater Management for Port Moresby, Papua New Guinea." National Conference Publication, Engineers Australia, Barton, Australia.

Carter, Ralf. 1990. "Hydraulic and Water Quality Studies, Erakor Lagoons and Port Vila Harbour, Vanuatu." SOPAC, Suva, Fiji.

Chaimanee, N. 2013. "Groundwater Development in the CCOP Countries: Challenges and Opportunities." Environmental Earth Sciences 70 (5).

Chan, T., H. Ross, S. Hoverman, and B. Powell. 2010. "Participatory Development of a Bayesian Network Model for Catchment-Based Water Resource Management." Water Resources Research 46: 1-12.

Chandran, Abirosh, and A. A. Mohamed Hatha. 2003. "Survival of Escherichia Coli in A Tropical Estuary." South Pacific Journal of Natural and Applied Sciences 21: 41-6.

Chang, C. 2012. "Cutting Edge Issues in Rheumatic Fever." Clinical Reviews in Allergy and Immunology 42 (3): 269-78.

Channnan, Lina. 2002. "Workshops on Water Quality Surveillance and Safety, Fiji Islands." SOPAC, Suva, Fiji.

Chew, R. T., III. 1999. "Environmental Problems on the Low Atolls of the Marshall Islands." Journal of Geoscience Education 47 (2): 143-9.

Choudhary, Ekta, Tai-Ho Chen, Colleen Martin, Sara Vagi, Joseph Roth Jr., Mark Keim, Rebecca Noe, Seiuli Elisapeta Ponausuia, Siitia Lemusu, Tesfaye Bayleyegn, and Amy Wolkin. 2012. "Public Health Needs Assessments of Tutuila Island, American Samoa, After the 2009 Tsunami." Disaster Medicine and Public Health Preparedness 6 (3): 209-16.

Cities Development Initiative for Asia. 2012. Pre-Feasibility Study for GSA Drainage and Flood Management Project. Pasig, Philippines: Cities Development Initiative for Asia.

Clarke, M., S. Feeny, and J. Donnelly. 2014. "Water, Sanitation and Hygiene Interventions in the Pacific: Defining, Assessing and Improving 'Sustainability."' European Journal of Development Research 26: 692-706.

Collier, Leslie. 1995. "Impact of Face-Washing on Trachoma." Lancet 345 (8950): 650-1.

Convard, Nancy S., Andrew Tomlinson, and Christopher Welch. 2005. Strategies for Preventing and Mitigating Land-Based Sources of Pollution to Trans-Boundary Water Resources in the Pacific Region. Apia, Samoa: SPREP.

Crennan, Leonie. 1996. Wet and Dry Conservancy: Politics and Practicalities of On-Site Sanitation. Hobart, Australia: University of Tasmania.

---. 2001. Integration of Social and Technical Science in Groundwater Monitoring and Management Groundwater Pollution Study on Lifuka, Ha'Apai, Tonga Recharge Study on Bonriki, South Tarawa, Kiribati. Paris: UNESCO.

-- . 2002. "Theme 3: Awareness." In Pacific Regional Consultation on Water in Small Island Countries: Papers and Proceedings, edited by Clive Carpenter, Jeffry Stubbs, and Marc Overmars, 81-102. Suva, Fiji: SOPAC.

- - . 2003. "Improving Community Participation in Water and Sanitation Projects in Pacific Island Countries." Development Bulletin 63, Australian National University, Canberra, Australia.

-- . 2004. "Tapping Connections Between People and Water." Miscellaneous report 577, SOPAC, Suva, Fiji.

- - . 2005. "Equitable Management of Water and Sanitation in Pacific Island Countries." SOPAC, Suva, Fiji. 
---. 2007a. Practical Training in Sustainable Sanitation for Tuvalu. Apia, Samoa: SPREP.

-- - 2007b. Sustainable Sanitation Manual and Construction Guidelines for a Waterless Composting Toilet. Apia, Samoa: SPREP.

Crennan, Leonie, and Greg Berry. 2002. Volume 3: A Synopsis of Information Relating to Waste Management, Pollution Prevention and Improved Sanitation with A Focus on Communities in the Pacific Islands Region: Issues for Community-Based Sustainable Resource Management and Conservation: Considerations for the Strategic Action Programme for the International Waters of the Pacific Small Island Developing States. Apia, Samoa: SPREP.

Cronin, S. J., and D. S. Sharp. 2002. "Environmental Impacts on Health from Continuous Volcanic Activity at Yasur (Tanna) and Ambrym, Vanuatu." International Journal of Environmental Health Research 12 (2): 109-23.

Daniell, K. A., I. White, N. Ferrand, I. Ribarova, P. Coad, J. E. Rougier, M. Hare, N. Jones, A. Popov, and D. Rollin. 2010. "Co-Engineering Participatory Water Management Processes: Theory and Insights from Australian and Bulgarian Interventions." Ecology and Society 15 (4): 11.

Davisson, M. Lee, Terry F. Hamilton, and Andrew F. B. Tompson. 2012. "Radioactive Waste Buried beneath Runit Dome on Enewetak Atoll, Marshall Islands." International Journal of Environment and Pollution 49 (3-4): 161-78.

Dawe, Paula. 2000. "Pipe Detection Surveys and Creation of a Water Utility Gis, Niue." SOPAC, Suva, Fiji.

- - . 2001. "Summary of SOPAC Water Demand Management and Conservation Activities in the Pacific Region." SOPAC, Suva, Fiji.

Deng Chenda, Ryan T. Bailey. 2017. "Assessing groundwater availability in the Maldives under future climate conditions." Hydrological Process. 31 (19):3334-3349

Dawe, Paula, and Harald Schölzel. 2000. "Hydraulic Network Modelling of the Rarotonga Water Supply System Cook Islands." SOPAC, Suva, Fiji.

Dengler, Lori, and Jane Preuss. 2003. "Mitigation Lessons from the July 17, 1998 Papua New Guinea Tsunami." Pure Applied Geophysics 160: 2001-31.

Denton, G. R., C. A. Emborski, N. C. Habana, and J. A. Starmer. 2014. "Influence of Urban Runoff, Inappropriate Waste Disposal Practices and World War II on the Heavy Metal Status of Sediments in the Southern Half of Saipan Lagoon, Saipan, CNMI." Marine Pollution Bulletin 81 (1): 276-81.

Denton, Gary R. W., and Carmen M. Sian-Denton. 2010. "Groundwater Monitoring on Guam: Management Responses to Recent Water Quality Violations." Groundwater Monitoring and Remediation 30 (2): 127-33.

Department of National Planning and Monitoring. 2015. "PNG National Water, Sanitation and Hygiene (WASH) Policy." Department of National Planning and Monitoring, Port Moresby, Papua New Guinea.

Depledge, Derrick. 1997. "Design Examples of Waterless Composting Toilets." Miscellaneous Report 249, SOPAC, Suva, Fiji.

Desmond, Yoli, Tom Tavala, and Marie Tiana Hakwa. 2004. Review of Environmental Legislation and Policies in Vanuatu. Apia, Samoa: SPREP.

Desowitz, R. S., and G. Barnish. 1986. "Entamoeba Polecki and Other Intestinal Protozoa in Papua New Guinea Highland Children." Annals of Tropical Medicine \& Parasitology 80: 399-402.

Detay, M., E. Alessandrello, P. Come, and I. Groom. 1989. "Groundwater Contamination and Pollution in Micronesia." Journal of Hydrology 112 (1-2): 149-70. 
Dethlefs, R. 1982. "The Trachoma Status and Blindness Rates of Selected Areas of Papua New Guinea in 197980." Australian Journal of Ophthalmology 10 (1): 13-8.

Dillaha, Theo A., and William J. Zolan. 1985. "Rainwater Catchment Water Quality in Micronesia." Water Research 19 (6): 741-6.

Dillon, Peter. 1997. "Groundwater Pollution by Sanitation on Tropical Islands." International Hydrological Programme, UNESCO, Paris.

Dornan, M. 2014. "Access to Electricity in Small Island Developing States of the Pacific: Issues and Challenges." Renewable and Sustainable Energy Reviews 31: 726-35.

Dray, Anne. 2006. "AtollGame: A Companion Modelling Experience in the Pacific." In Complex Science for a Complex World: Exploring Human Ecosystems with Agents, edited by Pascal Perez, and David Batten, 255-82. Canberra, Australia: ANU Press.

Drees-Gross, Franz R. 2015. Utility Services for the Development of Housing in Squatter/Informal Settlements. Washington, DC: World Bank.

Drew, W. M., K. C. Ewel, R. L. Naylor, and A. Sigrah. 2005. "A Tropical Freshwater Wetland: III. Direct Use Values and Other Goods and Services." Wetlands Ecology and Management 13 (6): 685-93.

Dudley, W. C., R. Whitney, J. Faasisila, S. Fonolua, A. Jowitt, and M. Chan-Kau. 2011. "Learning from the Victims: New Physical and Social Science Information About Tsunamis from Victims of the September 29, 2009 Event in Samoa and American Samoa." Earth-Science Reviews 107 (1-2): 201-6.

Duncan, L. E., and G. B. Bukenya. 1995. "Investigation of an Outbreak of Typhoid Fever in Two Settlements Near Port Moresby." Papua New Guinea Medical Journal 38 (1): 27-35.

Dunn, Eric G. 1981. Environmental Health in Rural Development and Food Hygiene. Nouméa, New Caledonia: SPC.

Dupon, Jean-François. 1993. Not Always a Pretty Face: Urban Growth Versus the Environment in the Pacific Islands. Apia, Samoa: SPREP.

Duwig, C., T. Becquer, B. E. Clothier, and M. Vauclin. 1998. "Nitrate Leaching Through Oxisols of the Loyalty Islands (New Caledonia) Under Intensified Agricultural Practices." Geoderma 84: 29-43.

Dworsky, Mike. 2002. "Theme 4: Technology." In Pacific Regional Consultation on Water in Small Island Countries: Papers and Proceedings, edited by Clive Carpenter, Jeffry Stubbs, and Marc Overmars, 103-36. Suva, Fiji: SOPAC.

Eason, R. J., and T. Tasman-Jones. 1985. "Resurgent Yaws and Other Skin Diseases in the Western Province of the Solomon Islands." Papua New Guinea Medical Journal 28 (4): 247-50.

Ebrahim, G. J. 2002. "Targeting Aid Programmes on Diseases That Cause Poverty." Journal of Tropical Pediatrics 48 (1): 2-4.

EC (European Commission). 2012. "Improved Drinking Water Supply for Kiritimati Island." International Cooperation and Development, European Commission, Brussels.

Ekali, Sarah. 2003. "Water and Sanitation in Rural Communities: A PNG Case Study." Development Bulletin 63, Australian National University, Canberra, Australia.

Engineers Australia. 1999. Improving Wastewater Treatment and Water Quality from Wells and Reservoirs. Barton, Australia: Engineers Australia.

Evans, Nathan. 2006. Natural Resources and the Environment in Fiji: A Review of Existing and Proposed Legislation. Apia, Samoa: SPREP. 
Evensen, C. I. 2010. "Sustainable Water Quality Management for Pacific Island Watersheds: Illustrations from American Samoa, Hawai'i, and Micronesia." In Sustainability Science for Watershed Landscapes, edited by James Roumasset, Kimberly M. Burnett, and Arsenio Molina Balisacan. Singapore: Institute of Southeast Asian Studies.

Fairbairn, Te'o lan, and Clem Tisdell. 1994. Applicability and Use of Natural Resource Accounting and Environmental Economics in Small Island Developing States. Apia, Samoa: SPREP.

Fakaosi, Sione, and Pelenatita Kara. 2004. Community Awareness, Engagement and Participatory Workshop Report. Apia, Samoa: SPREP.

Falkland, Tony. 1983. "Groundwater Resource Study of Christmas Island." Republic of Kiribati.

- - . 1992. Small Tropical Islands: Water Resources of Paradise Lost. Paris: UNESCO

---. 2002a. "From Vision to Action towards Sustainable Water Management in the Pacific. Theme 1: Overview Report Water Resources Management." Ecowise Environmental, Fyshwick, Australia.

--- 2002b. "Theme 1: Water Resources Management." In Pacific Regional Consultation on Water in Small Island Countries: Papers and Proceedings, edited by Clive Carpenter, Jeffry Stubbs, and Marc Overmars, 9-66. Suva, Fiji: SOPAC.

---. 2002c. Volume 2: A Synopsis of Information Relating to the Quality of Freshwater and Watershed Management Issues in the Pacific Islands Region: Issues for Community-Based Sustainable Resource Management and Conservation: Considerations for the Strategic Action Programme for the International Waters of the Pacific Small Island Developing States. Apia, Samoa: SPREP.

-- - 2011. Report on Water Security \& Vulnerability to Climate Change and Other Impacts in Pacific Island Countries and East Timor. Canberra, Australia: Department of Climate Change \& Energy Efficiency.

Falkland, T., and E. Custodio. 1991. "Hydrology and Water Resources of Small Islands: A Practical Guide." International Hydrological Programme, UNESCO, Paris.

Farley, Malcolm. 1999. "Managing Water Loss in Developing Countries." Water 21.

Feachem, Richard. 1973. "Recommendations for the Testing of Bacteriological Water Quality in Rural Papua New Guinea." Papua New Guinea Medical Journal.

Fiji Mineral Resources Department. 2015. "Fiji Groundwater." Brochure, Ministry of Lands and Mineral Resources, Suva, Fiji.

Filer, Colin, and Kay Kalim. 2003. "The Water Resource Management Regime in Papua New Guinea." Development Bulletin 64, Australian National University, Canberra, Australia.

Finau, S. A. 1987. "Community Priorities Following Disaster: A Case Study from Tonga." Social Science \& Medicine 24 (11): 961-6.

Finau, S., and S. A. Finau. 1983. "Better Accounting Improves Water Supply." World Health Forum 4 (2).

Finau, S. A., S. Fungalei, O. Isama'u, S. Finau, and P. Moa. 1986. "Environmental and Sanitary Conditions After a Cyclone in Tonga." Community Health Studies 10 (3): 336-43.

Fisher, P. J. 1987. "Artificial Marsh Treats Sewage in Papua New Guinea." Presented at Engineering Conference Darwin, "Developing Remote Areas," Darwin, Australia, May 11-15.

Fontaine, Jeffery. 1987. "Water Supply in Micronesia." American Water Works Association 79 (7): 64-9.

Fraser Thomas Partners. 2011. "South Tarawa Water and Sanitation Roadmap 2011-2030, Volume 1 Main Report." Fraser Thomas Partners, Papatoetoe, New Zealand. 
Frost, F. J., R. L. Calderon, and G. F. Craun. 1995. "Waterborne Disease Surveillance: Findings of Survey of State and Territorial Epidemiology Programs." Journal of Environmental Health 58: 6-11.

Fujioka, R. S. 2001. "Monitoring Coastal Marine Waters for Spore-Forming Bacteria of Faecal and Soil Origin to Determine Point from Non-Point Source Pollution." Water Science and Technology 44 (7): 181.

Fujioka, R., C. Sian-Denton, M. Borja, J. Castro, and K. Morphew. 1999. "Soil: The Environmental Source of Escherichia Coli and Enterococci in Guam's Streams." Journal of Applied Microbiology 85 (Suppl. 1): 83S-89S.

Fujita, Masafumi, Yoichi Ide, Daisaku Sato, Paul S. Kench, Yuji Kuwahara, Hiromune Yokoki, and Hajime Kayanne. 2014. "Heavy Metal Contamination of Coastal Lagoon Sediments: Fongafale Islet, Funafuti Atoll, Tuvalu." Chemosphere 95: 628-34.

Fujita, Masafumi, Jumpei Suzuki, Daisaku Sato, Yuji Kuwahara, Hiromune Yokoki, and Hajime Kayanne. 2013. "Anthropogenic Impacts on Water Quality of the Lagoonal Coast of Fongafale Islet, Funafuti Atoll, Tuvalu." Sustainability Science 8 (3): 381-90.

GEF (Global Environment Facility). 2004. "Integrated Water Resource Management Demonstration ProjectEnvironmental and Socio-Economic Protection in Fiji: Integrated Flood Management in the Nadi River Basin." Draft, GEF, Suva, Fiji.

-- . 2007. "Report on GEF Hotspot Workshop for Fiji." National Water Committee, Suva, Fiji.

GEF (Global Environment Facility), UNDP (United Nations Development Programme), SOPAC (Pacific Islands Applied Geoscience Commission), and UNEP (United Nations Environment Programme). 2007. National Integrated Water Resource Management Diagnostic Report: Kiribati. Washington, DC: Global Environment Fund.

GEF-IW:LEARN (Global Environment Facility International Waters Learning Exchange and Resource Network), Global Water Partnership-Caribbean (GWP-C), Global Environment Facility (GEF) Amazon Project, Caribbean Aqua-Terrestrial Solutions (CATS), and Environmental Health \& Sustainable Development Dept, Caribbean Public Health Agency (CARPHA). 2014. Rainwater Harvesting (RWH) Knowledge Network Forum Countries proceedings, Saint-Lucia.

Gerber, Frederica. 2010. "An Economic Assessment of Drinking Water Safety Planning Koror-Airai, Palau." SOPAC, Suva, Fiji.

Germani, Y., M. Morillon, E. Begaud, H. Dubourdieu, R. Costa, and J. Thevenon. 1994. "Two-Year Study of Endemic Enteric Pathogens Associated with Acute Diarrhea in New Caledonia." Journal of Clinical Microbiology 32 (6): 1532-6.

Ghassemi, F., A. J. Jakeman, and G. Jacobson. 1990. "Mathematical Modelling of Sea Water Intrusion, Nauru Island." Hydrological Processes 4 (3): 269-81.

GHD. 2014. "Kiribati Water and Sanitation Sector Situational Analysis and Scoping Mission Summary of InCountry Mission and Workshop Outcomes." GHD Pty Ltd, Canberra, Australia.

- - . 2015. Kiribati Water and Sanitation Sector Situational Analysis and Scoping Mission Situational Analysis and Needs Assessment." GHD Pty Ltd, Canberra, Australia.

Goldman, Charles R. 1976. "Ecological Aspects of Water Impoundment in the Tropics." Revista de Biologia Tropical 24 (Suppl. 1): 87-112.

Goodrich, J. A., and J. F. Mink. 1983. "Groundwater Management in the Guam Island Aquifer System." In Papers of the International Conference on Groundwater and Man, Sydney, 5-9 December 1983. Canberra, Australia: AGPS. 
GoRoF (Government of the Republic of Fiji). 2012. "Republic of Fiji National Climate Change Policy." Secretariat of the Pacific Community, Suva, Fiji.

GoRoF (Government of the Republic of Fiji), UNDP (United Nations Development Programme), GEF (Global Environment Facility), United Nations Environment Programme (UNEP), and SOPAC (Pacific Islands Applied Geoscience Commission). 2007. "National Integrated Water Resource Management Diagnostic Report Fiji Islands, Sustainable Integrated Water Resources and Wastewater Management in Pacific Island Countries." Miscellaneous Report 637, SOPAC, Suva, Fiji.

GoS (Government of Samoa). 2010. "Performance Audit Report on Monitoring of Drinking Water Quality." Samoa Audit Office, Apia, Samoa.

Green Climate Fund. 2015. "Consideration of Funding Proposals-Addendum Funding Proposal Package for FP008." Provisional Agenda Item 14, Livingstone, Republic of Zambia.

Greenwell, James, Judith McCool, Jacob Kool, and Mosese Salusalu. 2013. "Typhoid Fever: Hurdles to Adequate Hand Washing for Disease Prevention Among the Population of a Peri-Urban Informal Settlement in Fiji." Western Pacific Surveillance and Response Journal 4 (1): 41-5.

Griggs, John E., and Frank L. Peterson. 1993. "Ground-Water Flow Dynamics and Development Strategies at the Atoll Scale." Groundwater 31 (2): 209-20.

Guerrier, G., H. Foster, O. Metge, C. Chouvin, and M. Tui. 2012. "Cultural Contexts of Swine-Related Infections in Polynesia." Clinical Microbiology and Infection 19 (7): 595-9.

GWP Consultants, and SOPAC (Pacific Islands Applied Geoscience Commission). 2007. Integrated Water Resources Management in Pacific Island Countries: A Synopsis. Suva, Fiji: Dreamwise Limited.

Haddock, R. L., J. W. Gilmore, and F. Pimentel. 2002. "A Leptospirosis Outbreak on Guam Associated with an Athletic Event." Pacific Health Dialog 9 (2): 186-9.

Haddock, R. L., L. T. Truong, and T. S. Aguon. 2002. “Cholera Control on Guam, 2000.” Pacific Health Dialog 9 (2): 190-2.

Hadwen, Wade L., Morgan C. MacDonald, Annika Kearton, Mark Elliott, Terence Chan, and Kate Shields. 2016. Developing Climate Resilient Water, Sanitation and Hygiene (WaSH) in the Pacific. Brisbane, Australia: Griffith University.

Hadwen, Wade L., Bronwyn Powell, Morgan C. MacDonald, Mark Elliott, Terence Chan, Wolfgang Gernjak, and William G. L. Aalbersberg. 2015. "Putting WASH in the Water Cycle: Climate Change, Water Resources and the Future of Water, Sanitation and Hygiene Challenges in Pacific Island Countries." Journal of Water, Sanitation and Hygiene for Development 5 (2): 183-91. doi:10.2166/washdev.2015.133.

Haga, M., O. Hanada, N. Takahashi, N. Kusakabe, H. Gotoh, Y. Maeno, and M. Takezawa. 2012. “Development and Infrastructure of the Federated States of Micronesia." Island Sustainability 166: 169-79.

Hajkowicz, Stefan, and Petero Okotai. 2006. An Economic Valuation of Watershed Pollution in Rarotonga, the Cook Islands. Apia, Samoa: SPREP.

Halcrow, G., C. Rowland, J. Willetts, J. Crawford, and N. Carrard. 2010. "Resource Guide: Working Effectively with Women and Men in Water, Sanitation and Hygiene Programs: Learnings from Research on Gender Outcomes from Rural Water, Sanitation and Hygiene Projects in Vanuatu and Fiji." International Women's Development Agency and Institute for Sustainable Futures, University of Technology, Sydney, Australia.

Hall, J. J., J. A. Gillespie, A. Rosewell, and P. Mapira. 2013. "The Papua New Guinea Cholera Outbreak: Implications for PNG, Australia and the Torres Strait." Medical Journal of Australia 199 (9): 576-7. 
Hardy J. T., and S. A. Hardy. 1972. "Tidal Circulation and Sewage Pollution in a Tropical Marine Lagoon." Environmental Pollution 3 (3): 195-203.

Harris, M., D. Nako, T. Hopkins, D. M. Powell, C. Kenny, C. Carroll, and K. Carroll. 1992. "Skin Infections in Tanna, Vanuatu in 1989." Papua New Guinea Medical Journal 35 (2): 137-43.

Hasan, T. J., A. Hicking, and J. David. 2011. "Empowering Rural Communities: Simple Water Safety Plans." Water Science \& Technology: Water Supply 11 (3): 309-17.

Hassan, Abdul. 2005. "A Preliminary Study on the Supply of Low Cost Housing in Fiji." Presented at the Pacific Rim Real Estate Society 11th Annual Conference, Melbourne, Australia, January 23-27.

Hay, J. E., G. Sem. 2000. "Vulnerability and adaptation: evaluation and regional synthesis of national assessments of vulnerability and adaptation to climate change". South Pacific Regional Environment Programme, Apia, Samoa.

Heath, S. A., and B. H. Heath. 1973. "Trachoma and Other Eye Disease in a New Guinea Village." American Journal of Ophthalmology 75 (1): 121-9.

Heitz, L. F., S. Khosrowpanah, and J. Nelson. 2000. "Sizing of Surface Water Runoff Detention Ponds for Water Quality Improvement." Journal of the American Water Resources Association 36 (3): 541-8.

Hill, David. 2002. "Theme 5: Institutional Arrangements." In Pacific Regional Consultation on Water in Small Island Countries: Papers and Proceedings, edited by Clive Carpenter, Jeffry Stubbs, and Marc Overmars, 137-62. Suva, Fiji: SOPAC.

Hocking, B. 1977. "Recent Advances in Gastro-Enteritis." Papua New Guinea Medical Journal 20 (2): 71-7.

Holding, S., and D. M. Allen. 2015. "Risk to Water Security for Small Islands: An Assessment Framework and Application." Regional Environmental Change 16: 827-39.

Horak, Helena M., Joshua S. Chynoweth, Ward P. Myers, Jennifer Davis, Scott Fendorf, and Alexandria B. Boehm. 2010. "Microbial and Metal Water Quality in Rain Catchments Compared with Traditional Drinking Water Sources in the East Sepik Province, Papua New Guinea." Journal of Water and Health 8 (1): 126-38.

Horwood, P. F., D. J. Barrington, and A. R. Greenhill. 2013. "More Resources Need to Be Committed to the Provision of Safe Water Sources and Sanitation in Papua New Guinea." Papua New Guinea Medical Journal 56 (3-4): 103-9.

Horwood, Paul, and Andrew Greenhill. 2011. "Cholera in Papua New Guinea and the Importance of Safe Water Sources and Sanitation." Western Pacific Surveillance and Response Journal 3 (1): 3-5.

Horwood, P. F., D. Luang-Suarkia, S. Bebes, K. Boniface, S. S. Datta, P. M. Siba, and C. D. Kirkwood. 2012. "Short Report: Surveillance and Molecular Characterization of Group A Rotaviruses in Goroka, Papua New Guinea." American Journal of Tropical Medicine and Hygiene 87 (6): 1145-8.

Hoverman, S., H. Ross, T. Chan, B. Powell. 2011. "Social Learning Through Participatory Integrated Catchment Risk Assessment in the Solomon Islands." Ecology and Society 16 (2): 17.

Hunt, Bruce. 1979. "An Analysis of the Groundwater Resources of Tongatapu Island, Kingdom of Tonga." Journal of Hydrology 40 (1-2): 185-96.

Hunt, C. D., Jr., and F. L. Peterson. 1980. "Groundwater Resources of Kwajalein Island, Marshall Islands." Technical Report 126, Water Resources Research Center, University of Hawaii, Honolulu.

Hunter H2O, and Solomon Water. 2017. Solomon Water 30 Year Strategic Plan: Main Report. Honiara, Solomon Islands: Solomon Water. 
-- - 2018. Solomon Water 30 Year Strategic Plan: Recommendations and Implementation Plan. Honiara, Solomon Islands: Solomon Water.

Hutchings P., R. Franceys, S. Smits and S. Mekala. 2017. "Community Management of Rural Water Supply. Case Studies of Success from India." Earthscan Studies in Water Resource Management. London, UK.

Hutchinson, P. J. 1996. "Waste Management for the Island of Rarotonga, Cook Islands, South Pacific." Northeastern Geology and Environmental Sciences 18 (4): 307-13.

IGRAC (International Groundwater Resources Assessment Centre). 2016. Groundwater Monitoring in Small Island Developing States in the Pacific. Suva, Fiji: SPC.

Imo, T. S., T. Oomori, M. Toshihiko, and F. Tamaki. 2007. "The Comparative Study of Trihalomethanes in Drinking Waters." International Journal of Environmental Science and Technology 4 (16): 421-6.

Institute of Marine Resources. 2004. Priority Environmental Concerns in Fiji. Apia, Samoa: SPREP.

Island Friends Ltd. 2004. Cook Islands Priority Environmental Problems (PEC) Report: A Review and Assessment of the Priority Environmental Concerns. Apia, Samoa: SPREP.

Jacobson, G., and P. J. Hill. 1993. "Groundwater and the Rehabilitation of Nauru." In Collected Case Studies in Engineering Geology, Hydrogeology, and Environmental Geology, edited by G. McNally, M. J. Night, and R. Smith, 103-19. Sydney: Geological Society Australia.

Jarris, P., and K. Sellers. 2017. "The State Public Health Agencies." In Scutfield and Keck's Principles of Public Health. Clifton Park, NY: Delmar Cengage Learning.

Jenkins, Carol. 1993. "Changing Hygiene Behavior in Papua New Guinea." Papua New Guinea Medical Journal 38 (4): 320-4.

Jenkins, C., and P. Howard. 1992. "The Use of Ethnography and Structured Observations in the Study of Risk Factors for the Transmission of Diarrhea in Highland Papua New Guinea." Medical Anthropology 15 (1): 1-16.

Jenkins, Aaron P., Namrata Prasad, Lanieta Naucukidi, Varanisese Rosa, Shalini Pravin, Gandercillar Vosaki, Rina Kumar, Talitha Cambemaiwai, Mike Kama, Kylie M. Jenkins, Stacy Jupiter, John A. Crump, Edward K. Mulholland, Pierre Horwitz, and Richard Strugnell. 2015. "An Interdisciplinary Study of Typhoid Fever in Central Division, Republic of Fiji." Presented at the 9th International Conference on Typhoid and Invasive NTS Disease, Bali, Indonesia, May 1.

Johnson, E., R. Jim, and B. I. Pavlin. 2010. "Hepatitis A in Pohnpei State, Federated States of Micronesia. 20082009." Pacific Health Dialog 16 (1): 91-7.

Jones, Paul. 2011. "Urban Poverty in Pacific Towns and Cities and the Impact from the Global Financial Crisis: Insights from Port Moresby, Papua New Guinea." In Congress Papers from the Planning Institute of Australia 2011 National Congress, 43-59. Sydney: University of Sydney.

- - . 2012a. "Pacific Urbanisation and the Rise of Informal Settlements: Trends and Implications from Port Moresby." Urban Policy and Research 30 (2): 145-60.

- - . 2012b. "Searching for A Little Bit of Utopia-Understanding the Growth of Squatter and Informal Settlements in Pacific Towns and Cities." Australian Planner 49 (4): 327-38.

Jones, Paul, and John Lea. 2007. "What Has Happened to Urban Reform in the Island Pacific? Some Lessons from Kiribati And Samoa." Pacific Affairs 80 (3): 473-91.

Kamundi, Edith, Annika Kearton, Iwona Kolodziejczyk, Diana Gonzalez Botero, and Regina Souter. n.d. "IFM Formative Research." International WaterCentre, Brisbane, Australia. 
Kaspari, P., and S. Allen. 2008. "Environmentally Sound Approaches for Stormwater Management on Pacific Island Nations." Presented at the World Environmental and Water Resources Congress, Honolulu, May 12-6.

Kazadi, W. M., K. B. Asiedu, N. Agana, and O. Mitja. 2014. "Epidemiology of Yaws: An Update." Clinical Epidemiology 6: 119-28.

Keen, M. 2003. "Integrated Water Management in the South Pacific: Policy, Institutional and Socio-Cultural Dimensions." Water Policy 5 (2): 147-64.

Keim, Mark E. 2010. "Sea-Level-Rise Disaster in Micronesia: Sentinel Event for Climate Change?" Disaster Medicine and Public Health Preparedness 4 (1): 81-7.

Khatri, K. 2009. "Integrated Biosystems-Montfort Boys Town, Suva, Fiji Islands." In Technologies and Management for Sustainable Biosystems, edited by Jaya Nair, Christine Furedy, Chanakya Hoysala, and Horst Doelle, 253-63. Hauppauge, NY: Nova Science.

Khosrowpanah, S., and L. F. Heitz. 1987. "Use of Appropriate Technology in the Design of Water Systems in Micronesia." In Proceedings of the International Conference on Resource Mobilization for Drinking Water and Sanitation in Developing Nations, San Juan, Puerto Rico, May 1987, 399-406. New York: American Society of Civil Engineers.

King, Trevor. 2016. "Irrigated Ethnoagriculture, Adaptation and Development: A Pacific Case Study." Journal of Pacific Studies 36 (2): 184-212.

Kirk, Martyn D., Tom Kiedrzynski, Eliaser Johnson, Amato Elymore, and Iris Wainiqolo. 2005. "Risk Factors for Cholera in Pohnpei During an Outbreak in 2000: Lessons for Pacific Countries and Territories." Pacific Health Surveillance and Response 12 (2): 17-22.

Koda, Kazuhisa, Yuzo Manpuku, Tsutomu Kobayashi, Satoshi Ishida, Shuhei Yoshimoto, and Masaaki Okubo. 2013. "A Study of the Sealing Effect in the Observation Well of the Freshwater Lens at Laura Island, Republic of the Marshall Islands." Japan Agricultural Research Quarterly 47 (3): 257-72.

Kohlitz, Jeremy, Tasleem Hasan, Kamal Khatri, Arieta Sokota, Steven Iddings, Una Bera, and Rebecca Psutka. 2013. "Assessing Reported Use and Microbiological Performance of a Point-Of-Use Household Water Filter in Rural Fiji." Journal of Water, Sanitation and Hygiene for Development 3 (2): 207.

Kohlitz, J. P., and M. D. Smith. 2015. "Water Quality Management for Domestic Rainwater Harvesting Systems in Fiji." Water Science \& Technology: Water Supply 15 (1): 134-41.

Koshy, Kanayathu, Melchior Mataki, and Murari Lal. 2008. Sustainable Development-A Pacific Islands Perspective: A Report on Follow Up to the Mauritius 2005 Review of the Barbados Programme of Action. Suva, Fiji: Pacific Centre for Environment and Sustainable Development.

Kuberski, T. 1980. "Cholera on Nauru. Possible Non-Point Source Transmission." Medical Journal of Australia 2 (10): 563-4.

Kuberski, T., T. Flood, and T. Tera. 1979. "Cholera in the Gilbert Islands. I. Epidemiological Features." American Journal of Tropical Medicine and Hygiene 88 (2): 109-22.

Kuberski, T., A. Roberts, B. Linehan, R. N. Bryden, and M. Teburae. 1979. “Coconut Water As A Rehydration Fluid." New Zealand Medical Journal 90 (641): 98-100.

Kumar, Vinesh. 2010. "Water Management in Fiji." International Journal of Water Resources Development 26 (1): 81-96.

Kuruppu, N. 2009. "Adapting Water Resources to Climate Change in Kiribati: The Importance of Cultural Values and Meanings." Environmental Science and Policy 12 (7): 799-809. 
Kuruppu, Natasha, and Diana Liverman. 2011. "Mental Preparation for Climate Adaptation: The Role of Cognition and Culture in Enhancing Adaptive Capacity of Water Management in Kiribati." Global Environmental Change 21 (2): 657-69.

Kvandal, S. C., and F. H. Barrett. 1981. "Water System Planning in the Western Pacific." Presented at the Water Forum '81, San Francisco, CA, August 10-14.

Lal, Padma, and Meg Keen. 2002. Volume 5: Economic Considerations in Community-Based Project Planning and Implementation, Issues for Community-Based Sustainable Resource Management and Conservation: Considerations for the Strategic Action Programme for the International Waters of the Pacific Small Island Developing States. Apia, Samoa: SPREP.

Lal, Padma, Kalesoma Saloa, and Falealili Uili. 2006. Economics of Liquid Waste Management in Funafuti, Tuvalu. Apia, Samoa: SPREP.

Lal, Padma, Margaret Tabunakawai, and Sandeep K. Singh. 2007. Economics of Rural Waste Management in the Rewa Province and Development of a Rural Solid Waste Management Policy for Fiji. Apia, Samoa: SPREP.

Lal, Padma, and Lilieta Takau. 2006. Economic Costs of Waste in Tonga. Apia, Samoa: SPREP.

Lamour, Peter. 1997. "State, Society and Governance in Melanesia: Corruption and Governance in the South Pacific." Discussion Paper, Australian National University, Canberra, Australia.

Lane, A. G. 1967. "New Guinea Village Water Supplies: A Comparison of Faecal Pollution Levels in Wells and Traditional Supplies." Medical Journal of Australia 1 (8): 385-9.

Lane, Marcus B. 2006a. Coastal Governance in Solomon Islands: An Evaluation of the Strategic Governance Issues Relating to Coastal Management. Apia, Samoa: SPREP.

- - . 2006b. Evaluating the Governance of Coastal Resources and Environments in Vanuatu. Apia, Samoa: SPREP.

Larsen, F. T. 1995. "Typhoid Review, Enga Province, from 1986 to 1991." Papua New Guinea Medical Journal 38 (1): 20-6.

Lau, C. L., A. J. Dobson, L. D. Smythe, E. J. Fearnley, C. Skelly, A. C. A. Clements, S. B. Craig, S. D. Fuimaono, and P. Weinstein. 2012. "Leptospirosis in American Samoa 2010: Epidemiology, Environmental Drivers, and the Management of Emergence." American Journal of Tropical Medicine and Hygiene 86 (2): 309-19.

Leala-Gale, Christina. 2016. Pacific Islands Meteorological Services in Action. Apia, Samoa: SPREP.

Lehmann, D., A. Michael, M. Omena, A. Clegg, T. Lupiwa, R. C. Sanders, B. Marjen, P. Wai'in, A. Rongap, G. Saleu, P. Namuigi, M. Kakazo, S. Lupiwa, D. J. Lewis, and M. P. Alpers. 1999. "Bacterial and Viral Etiology of Severe Infection in Children Less Than Three Months Old in the Highlands of Papua New Guinea." Pediatric Infectious Disease Journal (10 Suppl): S50-5.

Lesley C. Ewing, and Costas E. Synolakis. 2010. "Community Resilience: Lessons from Recent Disasters." Coastal Engineering Proceedings 32: 1-13.

Lockwood, H., and S. Smits. 2011. Supporting Rural Water Supply: Moving towards a Service Delivery Approach. Warwickshire, UK: Practical Action Publishing.

Lovai, Narua. 2007. Water Use Survey Report, Barakau Village, Central Province, PNG. Apia, Samoa: SPREP.

Low, John. 1981. Urbanization and Its Effects on the South Pacific Environment. Apia, Samoa: SPREP.

Mabbett, A. N. 1975. "Water Quality of a Micronesian Atoll: Public Health Implications." Journal of Environmental Health 37 (4): 332-41. 
MacDonald, Morgan C., Terence Chan, Mark Elliott, Annika Kearton, Katherine F. Shields, Dani J. Barrington, Regina T. Souter, Bronwyn R. Powell, Jamie Bartram, and Wade L. Hadwen. 2017. "Temporal and Thematic Trends in Water, Sanitation and Hygiene (WaSH) Research in Pacific Island Countries: A Systematic Review." Journal of Water, Sanitation and Hygiene for Development 7 (4): 352-68.

MacGregor, J. P., and J. R. L. Read. 1969. "Village Water Supply Investigation, Territory of Papua and New Guinea." Engineering Geology 3 (3): 217-32.

Mansur, Aisha, Jesse Doyle, and Oleksiy Ivaschenko. 2017. "Social Protection and Humanitarian Assistance Nexus for Disaster Response: Lessons Learnt from Fiji's Tropical Cyclone Winston." Discussion Paper 1701, World Bank, Washington, DC.

Marlow, Chris. 2016. "FSM Pohnpei Water \& Sewerage Strategic Review Report: Strategic Review for Sewerage." PRIF (Pacific Region Infrastructure Facility), Sydney, Australia.

Martin, Timothy M., and David W. Watkins Jr. 2010. "An Analysis of Household Rainwater Harvesting Systems in Falelima, Samoa." Presented at the World Environmental and Water Resources Congress, Providence, RI, May 16-20.

Maskall, Ken. 2016. "UNICEF Pacific (Solomon Islands) WASH Strategy Paper." UNICEF Pacific Office, Suva, Fiji.

McCarthy, D. D., and N. Fitzgerald. 1956. "I. Habit, Habitat and Hyperfilariation in the Epidemiology of Filariasis in Western Samoa." Transactions of the Royal Society of Tropical Medicine and Hygiene 50 (1): 58-65.

McCarthy, D. D., M. J. Marples, D. F. Bacon, and N. Fitzgerald. 1955. "Researches in Western Samoa I. General Sanitation and Intestinal Parasites." Transactions of the Royal Society of Tropical Medicine and Hygiene 49 (1): 71-5.

McNamara, Karen E., and Leone E. Limalevu. 2015. "Holistic, Participatory and Strategic: A Vulnerability and Adaptation Assessment of Pacific Communities Faced with Climate Change." Journal of Pacific Studies 35 (3): 28-39.

McVicar, T. R., and P. N. Bierwirth. 2001. "Rapidly Assessing the 1997 Drought in Papua New Guinea Using Composite AVHRR Imagery." International Journal of Remote Sensing 22 (11): 2109-28.

Melrose, Wayne. 2000. "Pigs, Pole-Toilets, and Pek-Pek-A Parasitologist in Paradise." Australian Journal of Medical Science.

Merson, M. H., W. T. Martin, J. P. Craig, G. K. Morris, P. A. Blake, G. F. Craun, J. C. Feeley, J. C. Camacho, and E. J. Gangarosa. 1977. "Cholera on Guam: 1974 Epidemiologic Findings and Isolation of Non Toxinogenic Strains." American Journal of Epidemiology 105 (4): 349-61.

Messina, A. M., and T. W. Biggs. 2016. "Contributions of Human Activities to Suspended Sediment Yield During Storm Events from a Small, Steep, Tropical Watershed." Journal of Hydrology 538: 726-42.

Metutera, Taboia. 2002. "Theme 1: Water Resources Management Case Study: Water Management in Kiribati with Special Emphasis on Groundwater Development Using Infiltration Galleries." SOPAC, Suva, Fiji.

Ministry of the Environment, GoF (Government of Fiji). 2007. Fiji National Liquid Waste Management Strategy and Action Plan. Apia, Samoa: SPREP.

Mirti, Anumitra V., and Sarah Davies. 2005. "Drinking Water Quality in the Pacific Island Countries: Situation Analysis and Needs Assessment." Joint Contribution Report 181, SOPAC, Suva, Fiji. 
Miwatani, T., T. Honda, M. Higashisutsumi, R. Tanaka, Y. Sakaue, T. Nakabayashi, J. Igo, and K. J. R. Abaidoo. 1990. "Bacterial Aetiology of Infantile Diarrhoea in Papua New Guinea." Journal of Tropical Pediatrics 36 (3): 101-3.

Mogensen, Ulla. 1996. "Engineering Report for Nan Mand Water Supply System, Kitti Municipality Section II Federated States of Micronesia." SOPAC, Suva, Fiji.

Moglia, Magnus, Pascal Perez, and Stewart Burn. 2008a. "Urbanization and Water Development in the Pacific Islands." Development 51: 49-55.

---. 2008b. "Water Troubles in A Pacific Atoll Town." Water Policy 10 (6): 613-37.

- - . 2012. "Assessing the Likelihood of Realizing Idealized Goals: The Case of Urban Water Strategies." Environmental Modelling \& Software 35: 50-60.

Mohanty, Manoranjan. 2015. "Climate-Induced Disaster, Urban Flooding and Human Security in Pacific Towns and Cities." Journal of Pacific Studies 35 (3): 5-27.

Mommen, Brecht, Karen Humphries-Waa, and Stanley Gwavuya. 2017. "Does Women's Participation in Water Committees Affect Management and Water System Performance in Rural Vanuatu?" Waterlines 36 (3): 216-32.

Morahan, R. J. 1968. "Salmonella, Shigella and Enteropathogenic Escherichia Coli Isolations in the East and West Sepik Districts, Territory of Papua and New Guinea." Medical Journal of Australia 2 (10): 438 40.

Moriarty, Patrick, Stef Smits, John Butterworth and Richard Franceys. 2013. Trends in rural water supply: Towards a service delivery approach. Water Alternatives 6(3): 329-349

Mosley, Luke. 2005. "Water Quality of Rainwater Harvesting Systems." SOPAC, Suva, Fiji.

Mosley, Luke M., and William G. L. Aalbersberg. 2003. "Nutrient Levels in Sea and River Water Along the 'Coral Coast' of Viti Levu, Fiji." South Pacific Journal of Natural and Applied Sciences 21: 35-40.

Mosley, Luke, and Clive Carpenter. 2005. "Niue Coastal Water Quality and Groundwater Resources Assessment." SOPAC, Suva, Fiji.

Mosley, Luke M., and Donald S. Sharp. 2005. "The Hydrogen Sulphide (H2S) Paper-Strip Test." SOPAC, Suva, Fiji.

Mosley, Luke M., Donald S. Sharp, and Sarabjeet Singh. 2004. "Effects of A Tropical Cyclone on the Drinking Water Quality of a Remote Pacific Island." Disasters 28 (4): 405-17.

Mosley, Luke, Sarabjeet Singh, and Bill Aalbersberg. 2005. "Water Quality Monitoring in Pacific Island Countries." SOPAC, Suva, Fiji.

Mosley, Luke, and SOPAC (Pacific Islands Applied Geoscience Commission). 2003. "Drinking Water Quality on Vanua Levu, Fiji Islands Following Cyclone Ami." Trip Report 313, SOPAC, Suva, Fiji.

Motavalli, P. P., H. Discekici, and J. Kuhn. 2000. "The Impact of Land Clearing and Agricultural Practices on Soil Organic C Fractions and CO2 Efflux in the Northern Guam Aquifer." Agriculture, Ecosystems and Environment 79 (1): 17-27.

Mourits, Lineke. 1996. "An Assessment of Saltwater Intrusion in Babai Pits and Some Water Supply Projects on Makin, Butaritari and Abaiang, Republic of Kiribati." SOPAC, Suva, Fiji.

Mourits, Lineke J. M., and Prem B. Kumar. 1995. "Rainwater Utilization in Rural Fiji." Waterlines 14 (2): 8-10.

Mudaliar, M. M., C. Bergin, and K. MacLeod. "Drinking Water Safety Planning: A Practical Guide for Pacific Island Communities." SOPAC, Suva, Fiji. 
Murrell, T. G. C. 1983. “Pigbel in Papua New Guinea: An Ancient Disease Rediscovered.” International Journal of Epidemiology 12: 211-4.

Murrell, T. G. C., and P. D. Walker. 1991. "The Pigbel Story of Papua New Guinea." Transactions of the Royal Society of Tropical Medicine and Hygiene 85 (1): 119-22.

Mycoo, Michelle, and Michael G. Donovan. 2017. "A Blue Urban Agenda: Adapting to Climate Change in the Coastal Cities of Caribbean and Pacific Small Island Developing States." Inter-American Development Bank, Washington, DC. https://publications.iadb.org/handle/11319/8264.

Nakada, Satoshi, Yu Umezawa, Makoto Taniguchi, and Hiroya Yamano. 2012. "Groundwater Dynamics of Fongafale Islet, Funafuti Atoll, Tuvalu." Groundwater 50 (4): 639-44.

Narayan, S. A., J. L. Kool, M. Vakololoma, A. C. Steer, A. Mejia, A. Drake, A. Jenney, J. F. Turton, J. Kado, and L. Tikoduadua. 2009. "Investigation and Control of an Outbreak of Enterobacter Aerogenes Bloodstream Infection in a Neonatal Intensive Care Unit in Fiji." Infection Control \& Hospital Epidemiology 30 (8): 797-800.

Neelim, Ananta, and Joseph Vecci. 2013. Evaluation of the Solomon Islands Rural Development Program. Washington, DC: World Bank.

Nicholls, Stephen. 2004. The Priority Environmental Concerns Of Papua New Guinea. Apia, Samoa: SPREP.

Nielsen, Christian. 2003. "Rethinking Water Education in the Pacific." Development Bulletin 64, Australian National University, Canberra, Australia.

Nielsen, Christian, Hazel Clothier, Robbie Henderson, Jady Smith, and Jacob Zikuli. 2007. Mobilising People Towards Integrated Water Resources Management: A Guide to Community Action. Suva, Fiji: Live \& Learn Environmental Education.

Noshkin, V. E., and W. L. Robison. 1997. "Assessment of a Radioactive Waste Disposal Site at Enewetak Atoll." Health Physics 73 (1): 234-47.

Nurse, Leonard, and Roger McLean. 2014. "Small Islands." In Climate Change 2014: Impacts, Adaptation, and Vulnerability. Part B: Regional Aspects. Contribution of Working Group II to the Fifth Assessment Report to the Intergovernmental Panel on Climate Change, edited by V. R. Barros, et al.,1613-54. Cambridge, UK: Cambridge University Press.

Ohtsuka, R., T. Hongo, T. Kawabe, T. Suzuki, T. Inaoka, T. Akimichi, and H. Sasano. 1985. "Mineral Content of Drinking Water in Lowland Papua." Environment International 11 (6): 505-8.

Overmars, Marc. 2001. "Water Resources Assessment: Laura, Majuro, Marshall Islands." SOPAC, Suva, Fiji.

Overmars, Marc, and Sasha Beth Gottlieb. 2009. "Adapting to Climate Change in Water Resources and Water Services in Caribbean and Pacific Small Island Countries." SOPAC, Suva, Fiji.

Overmars, Marc, Brooke Yamakoshi, Andrew Dow, and Roger Singleton. 2016. "Community Drinking Water Safety and Security Planning in Pacific Island Countries." WASH Field Note, UNICEF, New York.

Owen, I. L. 2005. "Parasitic Zoonoses in Papua New Guinea." Journal of Helminthology 79 (1): 1-14.

Pacific WASH Coalition. 2009. "Framework for the Pacific WASH Coalition." Fiji School of Medicine, Suva, Fiji.

Pacific Water and Wastes Association. 2013. "Pacific Water and Wastewater Utilities Benchmarking Report." PRIF (Pacific Region Infrastructure Facility), Sydney, Australia.

Palmer, C. F. 1986. "Management Techniques to Optimize Repetitive Operations in Small Water Supply Schemes." Journal of the Institution of Water Engineers and Scientists 40 (3).

Paquette, Jessy, and John Lowry. 2012. "Flood Hazard Modelling and Risk Assessment in the Nadi River Basin, Fiji, Using GIS and MCDA." South Pacific Journal of Natural and Applied Sciences 30: 33-43. 
Passey, M. 1995a. "The New Problem of Typhoid Fever in Papua New Guinea: How Do We Deal with It?" Papua New Guinea Medical Journal 38 (4): 300-4.

- - . 1995b. "Social and Ecological Considerations in the Prevention of Enteric Infections." Papua New Guinea Medical Journal 38 (4): 257-61.

Paul, Benjamin. 1958. "The Role of Beliefs and Customs in Sanitation Programs." American Journal of Public Health and the Nation's Health 48 (11 Pt. 1): 1502-6.

Perez, J., F. Brescia, J. Becam, C. Mauron, and C. Goarant. 2011. "Rodent Abundance Dynamics and Leptospirosis Carriage in an Area of Hyper-Endemicity in New Caledonia." PLoS Neglected Tropical Diseases 5 (10): e1361. doi:10.1371/journal.pntd.0001361.

Perez, P., A. Dray, I. White, C. LePage, and T. Falkland. 2003. "Atollscape: Simulating Freshwater Management in Pacific Atolls: Spatial Processes and Time Dependence Issues." In Integrative Modelling of Biophysical, Social, and Economic Systems for Resource Management Solutions. International Congress on Modelling and Simulation, Townsville, Australia, 14-17 July 2003, edited by David A. Post, 514-18. Townsville, Australia: MSSANZ.

Perrocheau, A., and P. Perolat. 1997. "Epidemiology of Leptospirosis in New Caledonia (South Pacific): A OneYear Survey." European Journal of Epidemiology 13 (2): 161-7.

Poustie, M., R. R. Brown, A. Deletic, and F. J. De Haan. 2012. "Institutional Barriers to Advancing Sustainable Urban Water Management in Port Vila, Vanuatu." In WSUD 2012: Water Sensitive Urban DesignBuilding the Water Sensitive Community, 7th International Conference on Water Sensitive Urban Design, 885-92. Barton, Australia: Engineers Australia.

Poustie, M. S., and A. Deletic. 2014. "Modeling Integrated Urban Water Systems in Developing Countries: Case Study of Port Vila, Vanuatu." Ambio 43 (8): 1093-111.

Powell, Graham Bruce. 2004. Review of Natural Resources and Environmental Related Legislation-Phases 1 and 2. Apia, Samoa: SPREP.

Powell, Graham. 2006a. Analysis of Environment-Related Legislation in Tonga. Apia, Samoa: SPREP.

-- . 2006b. Inventory of Tonga's Environment-Related Laws. Apia, Samoa: SPREP.

-- . 2007. Analysis of Environment-Related Legis/ation in Niue. Apia, Samoa: SPREP.

Prasad, Namrata. 2015. "Case Control Study to Identify Risk Factors for Typhoid Fever in Central Division, Fiji: Preliminary Results." Presented at the 9th International Conference on Typhoid and Invasive NTS Disease, Bali, Indonesia, May 1.

Pritchard, D. I. 1995. "Gastrointestinal Nematodes: The Karkar Experience." Papua New Guinea Medical Journal 38 (4): 295-9.

Psutka, Rebecca, Patricia Priest, Tieren Davies, Teretia Rakunuea, Steven Iddings, and Andre Reiffer. 2013. "Assessing the Demographic, Behavioral and Environmental Characteristics and the Potential Effectiveness of a Household Water Filter in the Republic of Kiribati." Journal of Water, Sanitation and Hygiene for Development 3 (4): 530-40.

PWA (Pacific Water Association). n.d. "Water Governance: Pacific Islands." Apia, Samoa: PWA. https://www.pecc.org/resources/infrastructure-1/1109-water-governance-pacific-islands/file.

PWWA (Pacific Water and Wastewater Association). 2016. Benchmarking Report 2016-Pacific Water and Wastewater Association Five Years of Performance Assessment (2011-2015). Apia, Samoa: PWWA.

R\&M Engineering. 2014. "Yap State Public Service Corporation Final Draft Water System Master Plan." R\&M Engineering, Ketchikan, AK. 
Rajapakse, J. P., and R. A. Fenner. 2011. "Evaluation of Alternative Media for Pebble Matrix Filtration Using Clay Balls and Recycled Crushed Glass." Journal of Environmental Engineering 137 (6): 517-24.

Rajapakse, J. P., G. Madabhushi, R. Fenner, and C. Gallage. 2012. "Properties of Hand-Made Clay Balls Used As A Novel Filter Media." Geomechanics and Engineering 8 (6): 859-72.

Rao, Shiwangni, Mary Taylor, and Anjeela Jokhan. 2014. "In Vivo Screening of Salinity Tolerance in Giant Swamp Taro (Cyrtosperma Merkusii)." South Pacific Journal of Natural and Applied Sciences 32: 336.

Rapaport, M. 1990. "Population Pressure on Coral Atolls: Trends and Approaching Limits." Atoll Research Bulletin 340: 1-33.

Raturi, Atul. 2011. "Feasibility Study of a Solar Water Pumping System." Applied Solar Energy 47 (1): 11-3.

Reeve, P. A., and D. E. Dwyer. 1995. "An Outbreak of Typhoid Associated with A 'Lafet' in Vanuatu." Medical Journal of Australia 162 (1): 55-6.

Repič, J. 2011. "Appropriation of Space and Water in Informal Urban Settlements of Port Moresby, Papua New Guinea." Anthropological Notebooks 17 (3): 73-87.

Ricci, Giovanni, and David Scott. 1999. "Groundwater Potential Assessment of Rarotonga Coastal Plain." SOPAC, Suva, Fiji.

Roberts, S. 1992. “Coliform Bacteria from Aquatic Sources in Fiji." Journal of Applied Bacteriology 73 (3): $263-$ 8.

Roberts, A. B., J. R. Harris, T. Taitai, T. Taraam, J. Tekanene, A. Cati, T. Flood, and M. Teburae. 1979. “Cholera in the Gilbert Islands." American Journal of Tropical Medicine 28 (4): 685-91.

Robinson, D. C., B. Adriaans, R. J. Hay, and P. Yesudian. 1988. "The Clinical and Epidemiologic Features of Tropical Ulcer (Tropical Phagedenic Ulcer)." International Journal of Dermatology 27 (1): 49-53.

Robison, W. L., and V. E. Noshkin. 1999. "Radionuclide Characterization and Associated Dose from Long-Lived Radionuclides in Close-In Fallout Delivered to the Marine Environment at Bikini and Enewetak Atolls." Science of the Total Environment 237-238: 311-27.

Rogers, S., S. Paija, J. Embiap, and R. E. Pust. 1991. "Management of Common Potentially Serious Paediatric Illnesses by Aid Post Orderlies at Tari, Southern Highlands Province." Papua New Guinea Medical Journal 34 (2): 122-8.

Rosewell, Alexander, Benita Addy, Lucas Komnapi, Freda Makanda, Berry Ropa, Enoch Posanai, Samir Dutta, Glen Mola, Nicola Man, Anthony Zwi, and Chandini Raina Macintyre. 2012. "Cholera Risk Factors, Papua New Guinea, 2010." BMC Infectious Diseases 12 (1): 287.

Roy, P., and J. Connell. 1991. "Climatic Change and the Future of Atoll States." Journal of Coastal Research 7 (4): 1057-75.

Salim, M. R., F. Othman, Md. Imtiaj Ali, J. Patterson, and T. Hardy. 2002. "Application of Locally Available Materials for the Treatment of Organic Polluted Water." Water Science and Technology 46: 339-46.

Saunders, S. G., D. J. Barrington, S. Sridharan, S. Meo, W. Hadwen, K. F. Shields, R. Souter, and J. Bartram. 2016. "Addressing Water, Sanitation and Hygiene Challenges in Pacific Island Countries: A Participatory Systems Mapping Approach to Empower Informal Settlement Community Action." Habitat International 55: 159-66. doi:10.1016/j.habitatint.2016.03.010.

Schoeffel, P. 1984. "Dilemmas of Modernization in Primary Health Care in Western Samoa." Social Science and Medicine 19 (3): 209-16. 
Schölzel, Harald. 1998a. "Hydraulic Network Modelling of the Nukua'Lofa Water Supply System, Kingdom of Tonga." SOPAC, Suva, Fiji.

-- . 1998b. A Technical Appraisal of the Auki Water Supply System Malaita Island, Solomon Islands. New York: UNDP.

- - . 1998c. "Water Supply for Majuro, Republic of Marshall Islands: A Technical Appraisal for Feasible Options." SOPAC, Suva, Fiji.

- - . 1999. "Water Demand Management as a Tool to Mitigate Drought Impacts in Rural Water Supply Systems Report of Visit to Kadavu." SOPAC, Suva, Fiji.

Schölzel, Harald, and Rhonda Bower. 1999. "Small Scale Wastewater Treatment Plant Project Report on Project Criteria." SOPAC, Suva, Fiji.

Schölzel, Harald, and Giovanni Ricci. 1998. "Assessment of Water Resources Manono Island, Samoa." SOPAC, Suva, Fiji.

Schrecongost, Alyse, and Katherine Wong. 2015. Unsettled: Water and Sanitation in Urban Settlement Communities of the Pacific. Washington, DC: World Bank.

Schrecongost, Alyse, Katherine Wong, Penny Dutton, and Isabel Blackett. 2015. "Delivering Water and Sanitation to Melanesian Informal Settlements." Water: Journal of the Australian Water Association $42(3): 40-3$.

Scobie, H. M., E. Nilles, M. Kama, J. L. Kool, E. Mintz, K. A. Wannemuehler, T. B. Hyde, A. Dawainavesi, S. Singh, S. Korovou, K. Jenkins, and K. Date. 2014. "Impact of a Targeted Typhoid Vaccination Campaign Following Cyclone Tomas, Republic of Fiji, 2010." American Journal of Tropical Medicine and Hygiene 90 (6): 1031-8.

Scott, David. 2002. "Theme 2: Island Vulnerability." In Pacific Regional Consultation on Water in Small Island Countries: Papers and Proceedings, edited by Clive Carpenter, Jeffry Stubbs, and Marc Overmars, 6780. Suva, Fiji: SOPAC.

Scott, David, Marc Overmars, Tony Falkland, and Clive Carpenter. 2003. Pacific Dialogue on Water and Climate Synthesis Report. Suva, Fiji: South Pacific Applied Geoscience Commission.

Scott, David, Giovanni Ricci, and Tevita Fatai. 1999. "Groundwater Assessment Kingdom of Tonga." SOPAC, Suva, Fiji.

Seleganiu, Pisi. 2013. "Integrated Sustainable Wastewater Management (Ecosan) for Tuvalu: Results Report 2013." IWRM GEF Program, SPC, Nouméa, New Caledonia

Sharma, Shereen, Robert Smith, and Graeme Frost. 2001. "Water-Quality Analysis Manihiki Lagoon, Cook Islands." SOPAC, Suva, Fiji.

Shields, K. F., R. T. Souter, D. J. Barrington, S. Sridharan, S. G. Saunders, S. Meo, and J. Bartram. 2017. “Policy Brief: Enabling Systems that Support Sustainable, Effective and Inclusive WaSH Marketing Exchanges." International WaterCentre, Brisbane, Australia.

Simon, S. L., and J. C. Graham. 1996. "Dose Assessment Activities in the Republic of the Marshall Islands." Health Physics Society 71 (4): 438-56.

Singh, Reena B. K., Simon Hales, Neil de Wet, Rishi Raj, Mark Hearnden, and Phil Weinstein. 2001. "The Influence of Climate Variation and Change on Diarrheal Disease in the Pacific Islands." Environmental Health Perspectives 109 (2): 155-9.

Singh, Sarabjeet, and Luke M. Mosley. 2003. "Trace Metal Levels in Drinking Water on Viti Levu, Fiji Islands." South Pacific Journal of Natural and Applied Sciences 21: 31-4. 
SI RWASH (Solomon Islands Rural Water Sanitation and Hygiene). 2014. "Guidance Note on MHM in Schools." SI

RWASH.

https://sirwash.weebly.com/uploads/4/2/7/6/42764129/si_2014_guidance_note_on_mhm_in_sch ools.pdf.

Slack, Neale J., and Gurmeet Singh. 2016. "An Insight into Public Sector Readiness for Change-The Fiji Experience." Journal of Pacific Studies 36 (2): 41-66.

Smith, Alan. 2003. "Community Water Supply and Sanitation in East Timor." Development Bulletin 63, Australian National University, Canberra, Australia.

Smith, D. 1984. "Towards a National Policy for the Provision of Village Water Supplies in Papua New Guinea." Papua New Guinea Medical Journal 27 (3-4): 117-9.

Smith, W. J., Jr. 2008. "The Place of Rural, Remote and Least-Wealthy Small Islands in International Water Development: The Nexus of Geography-Technology Sustainability in Chuuk State, Federated States of Micronesia." Geographical Journal 174 (3): 251-68.

- - . 2009. "Improving Access to Safe Drinking Water in Rural, Remote and Least-Wealthy Small Islands: Non-Traditional Methods in Chuuk State, Federated States of Micronesia." International Journal of Environmental Technology and Management 10 (2): 167-89.

Soil Science Society of America. 2007. "Freshwater Supplies Threatened in Central Pacific." Soil Science Society of America, Madison, WI.

SOPAC (Pacific Islands Applied Geoscience Commission). 1998. "A Report on the International Conference on the Radiological Situation at the Atolls of Mururoa \& Fangataufa." SOPAC, Suva, Fiji.

-- . 2000. Guidelines for Water and Sanitation Utilities Risk Management Planning. Suva, Fiji: SOPAC.

- - . 2001. "Pacific Wastewater Framework for Action." SOPAC, Suva, Fiji.

-- - 2002. "From Vision to Action: Towards Sustainable Water Resources Management in the Pacific Kiribati." Country Briefing Paper, SOPAC, Suva, Fiji.

-- . 2004a. Harvesting the Heavens: A Manual for Participatory Training in Rainwater Harvesting. Suva, Fiji: SOPAC.

-- . 2004b. "Water and Sanitation Issues and Strategic Responses in the Pacific." Pacific Brief, SOPAC, Suva, Fiji.

- - . 2007. “National Integrated Water Resource Management Diagnostic Report.” SOPAC, Suva, Fiji.

-- . 2008a. A Gender Tale of Water and Energy. Suva, Fiji: SOPAC.

- - . 2008b. "National Water Apex Bodies-A Guide for Pacific Island Countries." SOPAC, Suva, Fiji.

---. 2008c. Sanitation, Hygiene and Drinking Water in the Pacific Island Countries: Converting Commitment into Action. Manila, Philippines: WHO.

SOPAC (South Pacific Applied Geoscience Commission), and ADB (Asian Development Bank). 2003. Pacific Regional Action Plan on Sustainable Water Management. Mandaluyong, Philippines: ADB.

Sotheeswaran, Subramanium, Vikashni Nand, Maata Matakite, and Koshy Kanayathu. 2011. "Moringa Oleifera and Other Local Seeds in Water Purification in Developing Countries." Research Journal of Chemistry and Environment 15 (2): 135-8.

Souter, R. T., D. J. Barrington, S. Sridharan, K. F. Shields, S. G. Saunders, S. Meo, and J. Bartram. 2017. "Research Brief: Fostering WaSH Marketing in Informal Melanesian Settlements." International Water Centre, Brisbane, Australia. 
South, G. Robin, Posa A. Skelton, Joeli Veitayaki, Alan Resture, Clive Carpenter, Craig Pratt, and Alena Lawedrau. 2004. "The Global International Waters Assessment for the Pacific Islands: Aspects of Transboundary, Water Shortage, and Coastal Fisheries Issues." Ambio 33 (1): 98-106.

SPREP (South Pacific Regional Environment Programme). 2000. Overview on Land-Based Pollutant Sources and Activities Affecting the Marine, Coastal, and Freshwater Environment in the Pacific Islands Region. Apia, Samoa: SPREP.

-- - 2004. Participatory Situation Analysis: Summary Report of Village Consultations in Niue. Apia, Samoa: SPREP.

---. 2016. Strengthening Environmental Impact Assessment Guidelines for Pacific Island Countries and Territories. Apia, Samoa: SPREP.

Spriggs, M. 1982. "Traditional Uses of Fresh Water in Papua New Guinea: Past Neglect and Future Possibilities." Presented at Traditional Conservation in Papua New Guinea, "Implications for Today," Port Moresby, Papua New Guinea, October 27-31, 1980.

Sridharan, S., D. J. Barrington, and S. G. Saunders. 2015. "Water Exchange Systems." In Routledge Handbook of Water and Health, edited by J. Bartram, R. Baum, P. A. Coclanis, D. M. Gute, D. Kay, S. McFadyen, K. Pond, W. Roberston, and M. J. Rouse, 498-506. London: Routledge

Sridharan, S., D. J. Barrington, K. F. Shields, S. G. Saunders, R. T. Souter, S. Meo, and J. Bartram. 2017. "Programming Brief: Towards WaSH Programs that Measure and Enhance Sustained Wellbeing." International Water Centre, Brisbane, Australia.

Stewart, B. J. 1993. "The Hydrology and Water Resources of Humid Northern Australia and Papua New Guinea." In Hydrology and Water Management in the Humid Tropics: Hydrological Research Issues and Strategies for Water Management, edited by M. Bonell, M. Hufschmidt, and J. Gladwell, 67-83. Cambridge: Cambridge University Press. doi:10.1017/СBO9780511564468.010.

Storch, Gregory, Robert Gunn, William Martin, Robert Pollard, and Susanne Sinclair. 1980. "Shigellosis in the Marshall Islands: Epidemiological Aspects of an Outbreak." American Journal of Tropical Medicine and Hygiene 29 (3): 456-63.

Storey, Donovan. 1998. "The Politics of Managing Urban Development in Pacific Island States." Journal of Pacific Studies 22: 61-80.

- - . 2003. "The Peri-Urban Pacific: From Exclusive to Inclusive Cities." Asia Pacific Viewpoint 44 (3): 25979.

- - . 2005. "Urban Governance in Pacific Island Countries: Advancing an Overdue Agenda." Discussion Paper, State Society and Governance in Melanesia, Australian National University, Canberra, Australia.

- - . 2011. "Mobility as Development Strategy: 57-The Case of the Pacific Islands." Asia-Pacific Population Journal 26 (4): 57-72.

Storey, Donovan, and Shawn Hunter. 2010. "Kiribati: An Environmental 'Perfect Storm.'” Australian Geographer 41 (2): 167-81.

Strugnell, Dick. 2015. "Use of Molecular Biology to Decipher Typhoid Transmission in Fiji." University of Melbourne, Doherty Institute, Melbourne, Australia.

Sundaresan, T. K., B. Grab, K. Uemura, and B. Cvjetanović. 1974. "Comparative Epidemiological Analysis of Sanitation and Immunization in the Control of Typhoid and Cholera." American Journal of Public Health 64 (9): 910-2. 
Szamier, Margot, Christine McMahon, and Kim Titcombe. 2016. Review of Gender and Infrastructure in the PRIF Agencies. Sydney, Australia: Asian Development Bank.

Terry, J. 2005. Hazard Warning! Hydrological Responses in the Fiji Islands to Climate Variability and Severe Meteorological Events. Wallingford, UK: IAHS.

Terry, James P., and Anthony C. Falkland. 2009. "Responses of Atoll Freshwater Lenses to Storm-Surge Overwash in the Northern Cook Islands." Hydrogeology Journal 18 (3): 749-59.

Terry, James P., and Kamal Khatri. 2009. "People, Pigs and Pollution-Experiences with Applying Participatory Learning and Action (PLA) Methodology to Identify Problems of Pig-Waste Management at the Village Level in Fiji." Journal of Cleaner Production 17 (16): 1393-1400.

Terry, J. P., and R. Raj. 1999. "Island Environment and Landscape Responses to 1997 Tropical Cyclones in Fiji." Pacific Science 53 (3): 257-72.

Terry, J. P., R. Raj, and R. A. Kostaschuk. 2001. "Links Between the Southern Oscillation Index and Hydrological Hazards on a Tropical Pacific Island." Pacific Science 55 (3): 275-83.

Thomas, Frank R. 2003. “Kiribati: 'Some Aspects of Human Ecology,' Forty Years Later." Atoll Research Bulletin 501: 1-40.

Thomas, Pamela. 2003. "Water, Governance and the Political Economy: Discussion and Recommendations from the Policy Round Table and Community Workshop." Development Bulletin 63, Australian National University, Canberra, Australia.

Toelkes, W. 1987. “The Ebeye Desalination Project-Total Utilization of Diesel Waste Heat." Desalination 66: 59-68.

Tonga. 1988. "Fifth Five-Year Development Plan 1986-1990." Annual Review of Population Law 15: 296-9.

Tran, D., P. Phongsavan, A. E. Bauman, D. Havea, and G. Galea. 2006. "Hygiene Behavior of Adolescents in the Pacific: Associations with Socio-Demographic, Health Behavior and School Environment." Asia Pacific Journal of Public Health 18 (2): 3-11.

Trépied, B. 2011. "The Water Pipes for the Tribes: Municipal Action, Colonization and Citizenship in New Caledonia." Revue d'histoire moderne et contemporaine.

- - . 2012. "Indigenous Struggles and Water Policies in Contemporary New Caledonia." Journal for the Study of Race, Nation and Culture 18 (4): 465-79.

Tuhaika, John A., Jr. 2007. "State-Owned Enterprises and the Principal-Agent Problem: A Case Study of the Solomon Islands Water Authority." Pacific Economic Bulletin 22 (2): 131-9.

Umezaki, Masahiro, Maireng J. Sengebau-Kinzio, Keiko Nakamura, Eden Ridep, Masafumi Watanabe, and Takehito Takano. 2007. "Household Risk Factors Associated with Dengue-Like Illness, Republic of Palau, 2000-2001." BioScience Trends 1 (1): 33-7.

UNEP (United Nations Environment Programme). 2002. A Directory of Environmentally Sound Technologies for the Integrated Management of Solid, Liquid and Hazardous Waste for Small Island Developing States (SIDS) in the Pacific Region. Nairobi, Kenya: UNEP.

UNESCO (United Nations Educational, Scientific and Cultural Organisation). 2003. "Pacific Regional Consultation on Water in Small Island Countries." 3rd World Water Forum proceedings, UNESCO, Paris.

---. 2015. Pacific Education for All. Paris: UNESCO. 
UN-GLAAS (UN-Water Global Analysis and Assessment of Sanitation and Drinking-Water). 2017. Financing Universal Water, Sanitation and Hygiene Under the Sustainable Development Goals. New York: UNGLAAS.

UNICEF (United Nations Children's Emergency Fund). n.d. "Fiji WaSH Resilience Policy Framework Review." UNICEF, New York.

---. 2013a. A Snapshot of Water and Sanitation in the Pacific 2013 Sub-Regional Analysis and Update. New York: UNICEF.

---. 2013b. Solomon Islands Child-Centered Disaster Risk Assessment Zero Draft for Input. New York: UNICEF.

- - . 2014. "Case Study of Menstrual Hygiene Management in Schools in Honiara and Guadalcanal Province: School Report." New York: UNICEF.

-- - 2015. Second Review of Community-Led Total Sanitation in the East Asia and Pacific Region. Bangkok, Thailand: UNICEF East Asia and Pacific Regional Office.

---. 2016a. Drinking Water Safety and Security Plan (DWSSP) Programme Overview. New York: UNICEF.

-- - 2016b. Sanitation, Drinking-Water and Health in Pacific Island Countries 2015 Update and Future Outlook. New York: UNICEF.

- - . 2017a. "WaSH Resilience: Concepts, Progress and Next Steps." UNICEF, New York.

- - . 2017b. "WaSH Strategy Note: Pacific Multi Country Programme (2018-2022)." UNICEF Pacific Office, Suva, Fiji.

UNICEF (United Nations Children's Emergency Fund), and GWP (Global Water Partnership). 2014. WaSH and Climate Resilient Development. New York: UNICEF.

UN-Habitat (United Nations Settlements Programme). 2015. Urbanization and Climate Change in Small Islands Developing States. Nairobi, Kenya: UN-HABITAT

USGS (United States Geological Survey). 2008. Ground Water on Tropical Pacific Islands-Understanding A Vital Resource. Reston, VA: USGS.

Vail, John. 2002. "The Family Health and Rural Improvement Program in Tari." Papua New Guinea Medical Journal 45 (1-2): 147-62.

Vallance, Roger J. 2012. "A Melanesian Research Methodology." Presented at Joint AARE/APERA Conference, Sydney, Australia.

van der Velde, M., S. R. Green, M. Vanclooster, and B. E. Clothier. 2007. "Sustainable Development in Small Island Developing States: Agricultural Intensification, Economic Development, and Freshwater Resources Management on the Coral Atoll of Tongatapu." Ecological Economics 61 (2-3): 456-68.

Vigneron, E. 1989. "The Epidemiological Transition in an Overseas Territory: Disease Mapping in French Polynesia." Social Science \& Medicine 29 (8): 913-22.

Vince, J. D. 1995. "Diarrhea in Children in Papua New Guinea." Papua New Guinea Medical Journal 38 (4): 262-71.

Vuki, V. C., S. Appana, M. R. Naqasima, and M. Vuki. 2000. "Vanuatu." In Seas at the Millennium-An Environmental Evaluation, Volume 2, edited by C. Sheppard. New York: Elsevier Science.

Vuki, V. C., L. P. Zann, M. Naqasima, and M. Vuki. 2000. "The Fiji Islands." In Seas at the Millennium-An Environmental Evaluation, Volume 2, edited by C. Sheppard. New York: Elsevier Science. 
Wairiu, Morgan, and Bronwyn Powell. 2006. Australian Water Research Facility Situation Analysis Report: Solomon Islands. Brisbane, Australia: International Water Centre.

Walker, lan. 2002. "Theme 6: Financing." In Pacific Regional Consultation on Water in Small Island Countries: Papers and Proceedings, edited by Clive Carpenter, Jeffry Stubbs, and Marc Overmars, 163-88. Suva, Fiji: SOPAC.

Wallace, Corey, and Ryan Bailey. 2014. "Sustainable Rainwater Catchment Systems for Micronesian Atoll Communities." Journal of the American Water Resources Association 51 (1): 185-99.

Walzer, P. D., F. N. Judson, K. B. Murphy, G. R. Healy, D. K. English, and M. G. Schultz. 1973. "Balantidiasis Outbreak in Truk." American Journal of Tropical Medicine and Hygiene 22 (1): 33-41.

Water Institute at UNC. 2017. "Solomon Islands Rural Water, Sanitation and Hygiene Baseline Report." UNICEF Pacific Office, Suva, Fiji.

WaterAid Australia, and IWC (International Water Center). 2008. Sharing Experiences: Sustainable Sanitation in South East Asia and the Pacific. Canberra, Australia: AusAID.

WaterAid. 2016. Solomon Islands WASH Sector Analysis. Canberra, Australia: Australian Aid.

Wen, Yuming. 2011. "Impacts of Human Activities on Groundwater Quality in Guam, Mariana Islands." International Journal of Environmental, Cultural, Economic, and Social Sustainability 7 (5): 243-56.

Werner, Adrian D., Hannah K. Sharp, Sandra C. Galvis, Vincent E. A. Post, and Peter Sinclair. 2017. "Hydrogeology and Management of Freshwater Lenses on Atoll Islands: Review of Current Knowledge and Research Needs." Journal of Hydrology 551: 819-44.

White, Ian. 2005. "Climatic and Human Influences on Water Resources in Low Atolls." Presented at the Climatic and Anthropogenic Impacts on the Variability of Water Resources Conference.

---. 2006. "Kiribati Water Governance Milestone Report 1: Activities 1 and 2." Centre for Resource and Environmental Studies College of Science, Australian National University, Canberra, Australia.

White, lan, and Tony Falkland. 2010. "Management of Freshwater Lenses on Small Pacific Islands." Hydrogeology Journal 18 (1): 227-46.

- - . 2012a. "Practical Responses to Climate Change: Developing National Water Policy and Implementation Plans for Pacific Small Island Countries." Presented at the Practical Responses to Climate Change National Conference, "Water and Climate: Policy Implementation Challenges," Canberra, Australia, May 1-3.

- - . 2012b. "Reducing Groundwater Vulnerability in Carbonate Island Countries in the Pacific." In Climate Change Effects on Groundwater Resources, edited by Holger Treidel, Jose Luis Martin-Bordes, and Jason J. Gurdak, 75-109. Boca Raton, FL: CRC Press.

White, I., A. Falkland, L. Crennan, P. Jones, B. Etuati, and E. Metai. 1999. Issues, Traditions and Conflicts in Groundwater Use and Management. Paris: UNESCO

White, I., A. Falkland, L. Crennan, T. Metutera, B. Etuati, P. Perez, and A. Dray. 2002. "Hydrology of and Conflicts Over Shallow Groundwater Use and Management in Low Coral Islands." Presented at the International Symposium on Low-lying Coastal Areas, Hydrology and Integrated Coastal Zone Management, Bremerhaven, Germany, September 9-12.

White, I., T. Falkland, T. Metutera, M. Katatia, T. Abete-Reema, M. Overmars, P. Perez, and A. Dray. 2008. "Safe Water for People in Low, Small Island Pacific Nations: The Rural-Urban Dilemma." Development 51 (2): 282-7. 
White, I., A. Falkland, M. Rebgetz, M. Overmars, M. Katatia, and T. Metutera. 2009. "Development of National Water Resources Policy and Implementation Plans for the Kiribati Adaptation Program." In Proceedings, 32nd Hydrology and Water Resources Symposium, 1603-18. Newcastle, Australia: Engineers Australia.

White, Ian, Tony Falkland, Pascal Perez, Anne Dray, Marc Overmars, Eita Metai, and Taboia Metutera. 2004. "Small Island Water Supplies and Sanitation as the Epitome of Sustainability." Presented at the International Conference on Water Sensitive Urban Design, "Cities as Catchments," Adelaide, Australia, November 21-25.

White, Ian, Tony Falkland, Pascal Perez, Anne Dray, Taboia Metutera, Eita Metai, and Marc Overmars. 2007 . "Challenges in Freshwater Management in Low Coral Atolls." Journal of Cleaner Production 15 (16):

White, Ian, Tony Falkland, Taboia Metutera, Eita Metai, Marc Overmars, Pascal Perez, and Anne Dray. 2007b. "Climatic and Human Influences on Groundwater in Low Atolls." Valdose Zone Journal 6 (3): 581-90.

White, Ian, Tony Falkland, Taboia Metutera, Eita Metai, Pascal Perez, and Anne Dray. 2003. "Issues in Water Management and Use in Pacific Atoll Communities." Development Bulletin 63, Australian National University, Canberra, Australia.

White, Ian, Tony Falkland, Taboia Metutera, Eita Metai, Pascal Perez, Anne Dray, and Marc Overmars. 2006. "Society-Water Cycle Interactions in the Central Pacific: Impediments to Meeting the UN Millennium Goals for Freshwater." Presented at the RIHN 1st International Symposium, "Water and Better Human Life in the Future," Kyoto, Japan, November 6-8.

WHO (World Health Organization). 2014. Investing in Water And Sanitation: Increasing Access, Reducing Inequalities. Geneva: WHO

WHO (World Health Organization), and UNICEF (United Nations Children's Emergency Fund). 2017. Progess on Drinking Water, Sanitation and Hygiene: Launch Version. Geneva, Switzerland: WHO.

WHO (World Health Organization) Regional Office for the Western Pacific, UNICEF (United Nations Children's Emergency Fund), Pacific Community Water and Sanitation Programme, and United Nations Human Settlements Programme. 2015. Sanitation, Drinking-Water and Health in Pacific Island Countries: 2015 Update and Future Outlook. Manila, Philippines: WHO Regional Office for the Western Pacific.

Willetts, Juliet, Sally Asker, Naomi Carrard, and Keren Winterford. 2014. "The Practice of a Strengths-Based Approach to Community Development in the Solomon Islands." Development Studies Research 1 (1): 354-67.

Wilson, Catherine. 2018. "Youth Employment Critical to Sustainable Development in Pacific Islands." IPS News Agency (blog), May 10. http://www.ipsnews.net/2014/10/youth-employment-critical-tosust.inable-development-in-pacific-islands/.

Winter, Stephen J. 1987. "The Evolution of Water Supplies on the Remote Islands of Truk State: Preserving Tradition and the Environment." Journal of the Washington Academy of Sciences 77 (4): 224-9.

Wohl, Ellen. 2006. "Human Impacts to Mountain Streams." Geomorphology 79: 217-48.

Wohlfahrt, D. J., and K. Kukyuwa. 1982. "Village Rural Water Supplies in the Western Highlands Province of Papua New Guinea." Papua New Guinea Medical Journal 25 (3): 168-72.

Wong, T. H. F., and R. R. Brown. 2009. "The Water Sensitive City: Principles for Practice." Water Science and Technology 60 (3): 673-82.

World Bank. 2014. Water Supply and Sanitation in Timor-Leste: Turning Finance into Services for the Future. Washington, DC: World Bank. 
- - . 2017. "International Development Association Project Appraisal Document on a Proposed Credit in the Amount of SDR 51.0 Million (US \$70 Million Equivalent) to the Independent State of Papua New Guinea for a Water Supply and Sanitation Development Project." Report PAD1746, World Bank, Washington, DC.

- - . 2011. "Project Appraisal Document on A Proposed Grant from the Global Environment Facility-Least Developed Country Fund in the Amount of US $\$ 3.0$ Million to the Republic of Kiribati for the Kiribati Adaptation Program-Phase III." Report 63874-KI, World Bank, Washington, DC.

---. 2013a. Water Supply and Sanitation in Papua New Guinea: Turning Finance into Services for the Future. Washington, DC: World Bank.

- - . 2013b. "The World Bank Water and Sanitation Program Institutional Evaluation of PNG'S Water and Sanitation Sector." Sector Financial Review Draft Report, World Bank, Washington, DC.

- - . 2014a. "The Independent State of Papua New Guinea Water, Sanitation and Hygiene Policy Development in Papua New Guinea: Synthesis Report of Technical Assistance." Water and Sanitation Program Report, World Bank, Washington, DC.

- - . 2014b. Papua New Guinea Sanitation, Water Supply and Hygiene in Urban Informal Settlements: Social Research Findings and Recommendations." Water and Sanitation Program Report, World Bank, Washington, DC.

- - . 2016. "Solomon Islands Rural Development Program." Project Performance Assessment Report, World Bank, Washington, DC.

WSP (Water and Sanitation Program). 2015. Turning Finance into Services for the Future-A Regional Synthesis of the Service Delivery Assessments for Water Supply and Sanitation in East Asia and the Pacific. Washington, DC: World Bank.

Wutich, Amber, Alexandra Brewis, Abigail M. York, and Rhian Stotts. 2013. "Rules, Norms, and Injustice: A Cross-Cultural Study of Perceptions of Justice in Water Institutions." Society and Natural Resources 26 (7): 1-15.

Wutich, A., A. C. White, D. D. White, K. L. Larson, A. Brewis, and C. Roberts. 2014. "Hard Paths, Soft Paths or No Paths? Cross-Cultural Perceptions of Water Solutions." Hydrology and Earth System Sciences 18, 109-20.

Wutich, A., A. M. York, A. Brewis, R. Stotts, and C. M. Roberts. 2012. "Shared Cultural Norms for Justice in Water Institutions: Results from Fiji, Ecuador, Paraguay, New Zealand, and the U.S." Journal of Environmental Management 113: 370-6.

Wyrsch, Michael, Keith Coakley, Neal Alexander, Gerard Saleu, John Taime, Mexy Kakazo, Peter Howard, and Deborah Lehmann. 1998. "Diarrhea Morbidity in Children in the Asaro Valley, Eastern Highlands Province, Papua New Guinea." Papua New Guinea Medical Journal 41 (1): 7-14.

Yagi, K. 1979. "Rural Housing for Future Development." International Journal for Housing Science and Its Applications.

Yeo, S. W., R. J. Blong, and K. J. McAneney. 2007. "Flooding in Fiji: Findings from A 100-Year Historical Series." Hydrological Sciences Journal 52 (5): 1004-15.

Zann, L. P., and V. Vuki. 2000. "The South Western Pacific Islands Region." In Seas at the Millennium-An Environmental Evaluation, Volume 2, edited by C. Sheppard, 705-22. New York: Elsevier Science.

Zeenat, A., A. A. M. Hatha, L. Viola, and K. Vipra. 2009. "Bacteriological Quality and Risk Assessment of the Imported and Domestic Bottled Mineral Water Sold in Fiji." Journal of Water and Health 7 (4): 6429. 
Zolan, William J. 1981. Metal Concentrations in Guam Urban Runoff. Mangilao, Guam: University of Guam. ADB (Asian Development Bank). 2013. Republic of Fiji: Urban Development Planning and Institutional Capacity Building. Mandaluyong, Philippines: ADB. 